\title{
PATH FOLLOWING METHODS FOR STEADY LAMINAR BINGHAM FLOW IN CYLINDRICAL PIPES *
}

\author{
Juan Carlos De Los Reyes ${ }^{1}$ and Sergio GonzÁlez ${ }^{1}$
}

\begin{abstract}
This paper is devoted to the numerical solution of stationary laminar Bingham fluids by path-following methods. By using duality theory, a system that characterizes the solution of the original problem is derived. Since this system is ill-posed, a family of regularized problems is obtained and the convergence of the regularized solutions to the original one is proved. For the update of the regularization parameter, a path-following method is investigated. Based on the differentiability properties of the path, a model of the value functional and a correspondent algorithm are constructed. For the solution of the systems obtained in each path-following iteration a semismooth Newton method is proposed. Numerical experiments are performed in order to investigate the behavior and efficiency of the method, and a comparison with a penalty-Newton-Uzawa-conjugate gradient method, proposed in [Dean et al., J. Non-Newtonian Fluid Mech. 142 (2007) 36-62], is carried out.
\end{abstract}

Mathematics Subject Classification. 47J20, 76A10, 65K10, 90C33, 90C46, 90C53.

Received June 15, 2007. Revised June 2nd, 2008.

Published online October 16, 2008.

\section{INTRODUCTION}

Bingham models are used to analyze flows of materials for which the imposed stress must exceed a critical yield stress to initiate motion, i.e., they behave as rigid bodies when the stress is low but flow as viscous fluids at high stress. Examples of Bingham fluids include tooth paste, water suspensions of clay or sewage sludge.

For the mathematical analysis of Bingham fluid flow we refer to [7,9-11,22]. In [22] the authors consider a variational formulation of the model and study qualitative properties of it. Existence and uniqueness of the solution and the structure of the flow are investigated. In [7] the authors further analyze the resulting inequality of the second kind and prove, among other results, the Lipschitz stability of the solution with respect to the plasticity threshold. Further in [4] and [9-11] the authors investigate the regularity of the solution for the cross section and cavity model, respectively.

Bingham fluid flow in cylindrical pipes has been numerically treated by different methodologies. In [13], Chapter $\mathrm{V}$, the authors propose a global $\varepsilon$-type regularization of the model and prove the convergence of the regularized solutions towards the original one. Direct regularization of the primal problem by twice differentiable

\footnotetext{
Keywords and phrases. Bingham fluids, variational inequalities of second kind, path-following methods, semi-smooth Newton methods.

* Research partially supported by DAAD, EPN Quito and TU Berlin joint project: "Ph.D. Programme in Applied Mathematics".

${ }^{1}$ Research Group on Optimization, Departmento de Matemática, EPN Quito, Ecuador. jcdelosreyes@math.epn.edu.ec;

sgonzalez@math.epn.edu.ec
} 
functions has also been considered in [23] in combination with Newton methods. Although this type of regularization allows the direct use of second order methods, important discrepancies of the regularized problem with respect to properties of the original model arise (cf. [6], p. 39).

An alternative to the direct regularization of the primal problem consists in the so-called multiplier approach. In [13], the authors analyze the existence of multipliers by using duality theory and propose an Uzawa-type algorithm for its numerical solution. Also by using duality theory, augmented Lagrangian methods are proposed in $[18,19]$ and the unconditional convergence of the method is proven (see [19], Thm. 4.2). In the recent paper [6], the authors make a review of existing numerical approaches and propose a penalty-Newton-Uzawa conjugate gradient method for the solution of the problem. This approach is compared numerically with our method in Section 5 .

In this paper, we consider a Tikhonov regularization of the dual problem, which by duality theory implies a local regularization of the original one. The proposed local regularization allows the application of semismooth Newton methods and leads directly to a decoupled system of equations to be solved in each semismooth Newton iteration. This constitutes an important difference with respect to other primal-dual second order approaches (see e.g. [6]), where an additional method has to be used in order to obtain a decoupled system, at the consequent computational cost.

For the update of the regularization parameter a path following method is proposed and analyzed. The differentiability of the path and of the path value functional are studied. A model function that preserves the main properties of the value functional is proposed and a correspondent algorithm developed.

After discretization in space, each regularized problem is solved by using a semismooth Newton method. These type of methods have been successfully applied to infinite dimensional complementarity problems like the Signorini or contact problem (see [14,20,24,25]), image restoration (see [16]), optimal control problems (see $[5,17]$ ), and, in general, to infinite dimensional optimization problems (see $[16,17,19,26]$ ).

Path-following strategies together with semismooth Newton methods have been investigated in [14,15,25] for variational inequalities of the first kind and constrained optimal control problems. These cases involve unilateral pointwise constraints on the state variable, which are regularized by a Moreau-Yosida technique.

Differently from $[14,15]$, our problem involves a variational inequality of the second kind. As a result, and in contrast to unilateral pointwise constrained problems, pointwise constraints on the Euclidean norm of the velocity gradient have to be considered. This fact adds new difficulties to the path analysis. In particular, extra regularity estimates for the regularized solutions have to be obtained in order to get differentiability of the path.

Let us mention that, although the method developed in this article is concerned with Bingham fluid flow, the results can be extended to other variational inequalities of the second kind as well.

The paper is organized as follows. In Section 2 the original problem is stated and, using Fenchel's duality theory, a necessary condition is derived. Since the characterizing system for the original problem is ill-posed, a family of regularized problems is introduced and the convergence of the regularized solutions to the original one is proved. In Section 3, the path value functional is introduced and the differentiability of the path and the value functional is investigated. A model function which preserves the qualitative properties of the path-value functional is constructed and an iterative algorithm is proposed. In Section 4 a semismooth Newton method to solve the complementarity system for each regularized problem is stated. In Section 5, numerical experiments which show the main features of the proposed algorithm are presented.

\section{Problem Statement AND Regularization}

Let $\Omega$ be a bounded domain in $\mathbb{R}^{2}$, with Lipschitz boundary $\Gamma$, and let $f \in L^{2}(\Omega)$. We are concerned with the following variational inequality of the second kind: find $y \in H_{0}^{1}(\Omega)$ such that

$$
a(y, v-y)+g j(v)-g j(y) \geq(f, v-y)_{2}, \quad \text { for all } v \in H_{0}^{1}(\Omega),
$$

where $a(y, v):=\mu \int_{\Omega}\langle\nabla y(x), \nabla v(x)\rangle \mathrm{d} x, j(v):=\int_{\Omega}|\nabla v(x)| \mathrm{d} x$ and $(\cdot, \cdot)_{2}$ stands for the scalar product in $L^{2}(\Omega)$. The scalar product in $\mathbb{R}^{N}$ and the Euclidean norm are denoted by $\langle\cdot, \cdot\rangle$ and $|\cdot|$, respectively. $(\cdot, \cdot)_{X}$ stands 
for the scalar product in a Hilbert space $X$, and $\|\cdot\|_{X}$ for its associated norm. The duality pairing between a Banach space $Y$ and its dual $Y^{*}$ is represented by $\langle\cdot, \cdot\rangle_{Y^{*}, Y}$. Besides that, we will use the bold notation $\mathbf{L}^{2}(\Omega):=L^{2}(\Omega) \times L^{2}(\Omega)$.

Inequality (2.1) models the stationary flow of a Bingham fluid in a pipe of cross section $\Omega$ (see $[7,13,22]$ ). The variable $y(x)$ stands for the velocity at $x \in \Omega, f(x)$ for the linear decay of pressures, $\mu$ for the viscosity and $g$ for the plasticity threshold of the fluid (yield stress).

Problem (2.1) corresponds to the necessary condition of the following unconstrained minimization problem,

$$
\min _{y \in H_{0}^{1}(\Omega)} J(y):=\frac{1}{2} a(y, y)+g j(y)-(f, y)_{2} .
$$

Remark 2.1. It can be shown (cf. [12], Thm. 6.1) that there exists a unique solution $\bar{y} \in H_{0}^{1}(\Omega)$ to problem $(\mathcal{P})$. Moreover, if $\Omega$ has a sufficiently regular boundary, it follows that $\bar{y} \in H^{2}(\Omega) \cap H_{0}^{1}(\Omega)$, see [4], Theorem 15 .

\subsection{The Fenchel dual}

In this section, we obtain the dual problem of $(\mathcal{P})$ by using Fenchel's duality in infinite-dimensional spaces (see [8]). Let us start by defining the functionals $\mathcal{F}: H_{0}^{1}(\Omega) \rightarrow \mathbb{R}$ by $\mathcal{F}(y):=\frac{1}{2} a(y, y)-(f, y)_{2}$ and $\mathcal{G}:$ $\mathbf{L}^{2}(\Omega) \rightarrow \mathbb{R}$ by $\mathcal{G}(q):=g \int_{\Omega}|q(x)| \mathrm{d} x$. It can be easily verified that these two functionals are convex, continuous and proper. We also define the operator $\Lambda \in \mathcal{L}\left(H_{0}^{1}(\Omega), \mathbf{L}^{2}(\Omega)\right)$ by $\Lambda v:=\nabla v$. Thanks to these definitions, we may rewrite problem $(\mathcal{P})$ as

$$
\inf _{y \in H_{0}^{1}(\Omega)}\{\mathcal{F}(y)+\mathcal{G}(\Lambda y)\}
$$

Following [8], pp. 60-61, the associated dual problem of (2.2) is given by

$$
\sup _{q \in \mathbf{L}^{2}(\Omega)}\left\{-\mathcal{F}^{*}\left(-\Lambda^{*} q\right)-\mathcal{G}^{*}(q)\right\}
$$

where $\Lambda^{*} \in \mathcal{L}\left(\mathbf{L}^{2}(\Omega), H^{-1}(\Omega)\right)$ is the adjoint operator of $\Lambda$, and $\mathcal{F}^{*}: H^{-1}(\Omega) \rightarrow \mathbb{R}$ and $\mathcal{G}^{*}: \mathbf{L}^{2}(\Omega) \rightarrow \mathbb{R}$ denote the convex conjugate functionals of $\mathcal{F}$ and $\mathcal{G}$, respectively.

We recall that given a Hilbert space $H$ and a convex function $\varphi: H \rightarrow \mathbb{R} \cup\{-\infty,+\infty\}$, the convex conjugate functional $\varphi^{*}: H^{*} \rightarrow \mathbb{R} \cup\{-\infty,+\infty\}$ is defined by

$$
\varphi^{*}\left(v^{*}\right)=\sup _{v \in V}\left\{\left\langle v^{*}, v\right\rangle-\varphi(v)\right\}, \text { for } v^{*} \in V^{*}
$$

Thus, we have that

$$
\begin{aligned}
\mathcal{F}^{*}\left(-\Lambda^{*} q\right) & =\sup _{v \in H_{0}^{1}(\Omega)}\left\{\left\langle-\Lambda^{*} q, v\right\rangle_{H^{-1}(\Omega), H_{0}^{1}(\Omega)}-\frac{1}{2} a(v, v)+(f, v)_{2}\right\} \\
\mathcal{G}^{*}(q) & =\sup _{p \in \mathbf{L}^{2}(\Omega)}\left\{(q, p)_{\mathbf{L}^{2}(\Omega)}-g \int_{\Omega}|p(x)| \mathrm{d} x\right\} .
\end{aligned}
$$

Note that in (2.3) we have already identified $\mathbf{L}^{2}(\Omega)$ with its dual.

Now, let us calculate $\mathcal{F}^{*}\left(-\Lambda^{*} q\right)$. Let $q \in \mathbf{L}^{2}(\Omega)$ be given. From (2.4), we obtain that

$$
\mathcal{F}^{*}\left(-\Lambda^{*} q\right)=\sup _{v \in H_{0}^{1}(\Omega)}\left\{-(q, \Lambda v)_{\mathbf{L}^{2}(\Omega)}-\frac{1}{2} a(v, v)+(f, v)_{2}\right\},
$$


which implies, since $\left\{-(q, \Lambda v)_{\mathbf{L}^{2}(\Omega)}-\frac{1}{2} a(v, v)+(f, v)_{2}\right\}$ is a concave quadratic functional in $H_{0}^{1}(\Omega)$, that the supremum is attained at $v(q) \in H_{0}^{1}(\Omega)$ satisfying

$$
a(v(q), z)+(q, \Lambda z)_{\mathbf{L}^{2}(\Omega)}-(f, z)_{2}=0, \text { for all } z \in H_{0}^{1}(\Omega) .
$$

Using (2.6) with $z=v(q)$, we obtain that

$$
\mathcal{F}^{*}\left(-\Lambda^{*} q\right)=-(q, \nabla v(q))_{\mathbf{L}^{2}(\Omega)}-\frac{1}{2} a(v(q), v(q))+(f, v(q))_{2}=\frac{1}{2} a(v(q), v(q)) .
$$

Lemma 2.1. The expression

$$
(\bar{q}, p)_{L^{2}(\Omega)} \leq g \int_{\Omega}|p(x)| \mathrm{d} x, \quad \text { for all } p \in \boldsymbol{L}^{2}(\Omega)
$$

is equivalent to

$$
|\bar{q}(x)| \leq \text { g a.e. in } \Omega .
$$

Proof. Let us start by showing that (2.8) implies (2.9). Assume that (2.9) does not hold, i.e., assume that $S:=\{x \in \Omega: g-|\bar{q}(x)|<0$ a.e. $\}$ has positive measure. Choosing $\tilde{p} \in \mathbf{L}^{2}(\Omega)$ such that

$$
\tilde{p}(x):=\left\{\begin{array}{ccc}
\bar{q}(x) & \text { in } & S \\
0 & \text { in } & \Omega \backslash S
\end{array}\right.
$$

leads to

$$
g \int_{\Omega}|\tilde{p}(x)| \mathrm{d} x-\int_{\Omega}\langle\bar{q}(x), \tilde{p}(x)\rangle \mathrm{d} x=\int_{S}(g-|\bar{q}(x)|)|\bar{q}(x)| \mathrm{d} x<0
$$

which is a contradiction to (2.8). Conversely, due to the fact that $|\bar{q}(x)| \leq g$ a.e. in $\Omega$ and thanks to the Cauchy-Schwarz inequality, we obtain, for an arbitrary $p \in \mathbf{L}^{2}(\Omega)$, that

$$
g \int_{\Omega}|p(x)| \mathrm{d} x-\int_{\Omega}\langle\bar{q}(x), p(x)\rangle \mathrm{d} x \geq \int_{\Omega}(g-|\bar{q}(x)|)|p(x)| \mathrm{d} x \geq 0 .
$$

Lemma 2.1 immediately implies that

$$
\mathcal{G}^{*}(q)= \begin{cases}0 & \text { if }|q(x)| \leq g, \text { a.e. in } \Omega \\ +\infty & \text { otherwise. }\end{cases}
$$

Thus, using (2.7) and (2.10) in (2.3) we obtain the dual problem

$$
\left\{\begin{array}{l}
\sup _{|q(x)| \leq g} J^{*}(q):=-\frac{1}{2} a(v(q), v(q)) \\
\text { where } v(q) \text { satisfies } \\
a(v(q), z)-(f, z)_{2}+(q, \Lambda z)_{\mathbf{L}^{2}(\Omega)}=0, \text { for all } z \in H_{0}^{1}(\Omega) .
\end{array}\right.
$$

Due to the fact that both $\mathcal{F}$ and $\mathcal{G}$ are convex and continuous, [8], Theorem 4.1, p. 59, and [8], Remark 4.2, p. 60 , imply that no duality gap occurs, i.e.,

$$
\inf _{y \in H_{0}^{1}(\Omega)} J(y)=\sup _{\substack{\left.|q(x)| \leq g \\ a(v, z)+(q, \nabla z) \mathbf{L}^{2}(\Omega)=(f, z)\right)_{2}}} J^{*}(q),
$$

and that the dual problem $\left(\mathcal{P}^{*}\right)$ has at least one solution $\bar{q} \in \mathbf{L}^{2}(\Omega)$. 
Next, we will characterize the solutions of the primal and dual problems. From Fenchel's duality theory (see [8], Eqs. (4.22)-(4.25), p. 61) the solutions $\bar{y}$ and $\bar{q}$ satisfy the following extremality conditions:

$$
\begin{aligned}
-\Lambda^{*} \bar{q} & \in \partial \mathcal{F}(\bar{y}), \\
\bar{q} & \in \partial \mathcal{G}(\nabla \bar{y}) .
\end{aligned}
$$

Let us analyze (2.12). Since $\mathcal{F}$ is Gateaux differentiable in $\bar{y}$, [8], Proposition 5.3, p. 23, implies that $\partial \mathcal{F}(\bar{y})=$ $\left\{\mathcal{F}^{\prime}(\bar{y})\right\}$. Thus, we have that $(2.12)$ can be equivalently expressed as the following equation

$$
a(\bar{y}, v)-(f, v)_{2}+(\bar{q}, \nabla v)_{\mathbf{L}^{2}(\Omega)}=0, \text { for all } v \in H_{0}^{1}(\Omega) .
$$

On the other hand, from (2.13) and the definition of the subdifferential it follows that

$$
g\left(\int_{\Omega}|\nabla \bar{y}(x)| \mathrm{d} x-\int_{\Omega}|p(x)| \mathrm{d} x\right) \leq(\bar{q}, \nabla \bar{y}-p)_{\mathbf{L}^{2}(\Omega)}, \text { for all } p \in \mathbf{L}^{2}(\Omega) .
$$

Then, for $p=0$, we obtain that

$$
g \int_{\Omega}|\nabla \bar{y}(x)| \mathrm{d} x \leq(\bar{q}, \nabla \bar{y})_{\mathbf{L}^{2}(\Omega)},
$$

which implies, since $|\bar{q}(x)| \leq g$ a.e. in $\Omega$ and by Lemma (2.1), that

$$
g \int_{\Omega}|\nabla \bar{y}(x)| \mathrm{d} x=(\bar{q}, \nabla \bar{y})_{\mathbf{L}^{2}(\Omega)} .
$$

This last expression is equivalent to

$$
\left\{\begin{array}{l}
\nabla \bar{y}(x)=0 \quad \text { or } \\
\nabla \bar{y}(x) \neq 0, \quad \text { and } \quad \bar{q}(x)=g \frac{\nabla \bar{y}(x)}{|\nabla \bar{y}(x)|} .
\end{array}\right.
$$

Lemma 2.2. Equations (2.9) and (2.15) can be equivalently expressed as the following equation

$$
\max (\sigma g,|\sigma \bar{q}(x)+\nabla \bar{y}(x)|) \bar{q}(x)=g(\sigma \bar{q}(x)+\nabla \bar{y}(x)), \text { a.e. in } \Omega \text {, for all } \sigma>0 \text {. }
$$

Proof. We start by showing that (2.16) implies (2.9) and (2.15). From (2.16) it follows that

$$
\bar{q}(x)=g \frac{\sigma \bar{q}(x)+\nabla \bar{y}(x)}{\max (\sigma g,|\sigma \bar{q}(x)+\nabla \bar{y}(x)|)}, \text { a.e. in } \Omega,
$$

which immediately implies (2.9). Let us split $\Omega$ into the two following disjoint sets:

$$
\{x \in \Omega: \sigma g \geq|\sigma \bar{q}(x)+\nabla \bar{y}(x)|\} \text { and }\{x \in \Omega: \sigma g<|\sigma \bar{q}(x)+\nabla \bar{y}(x)|\} .
$$

On the set $\{x \in \Omega: \sigma g \geq|\sigma \bar{q}(x)+\nabla \bar{y}(x)|\}$, we have that $g(\sigma \bar{q}(x)+\nabla \bar{y}(x))-\sigma g \bar{q}(x)=0$, and thus $\nabla \bar{y}(x)=0$.

To see that $\nabla \bar{y}(x) \neq 0$ on the set $\{x \in \Omega: \sigma g<|\sigma \bar{q}(x)+\nabla \bar{y}(x)|\}$, we assume the opposite and immediately obtain that $g<|\bar{q}(x)|$, which contradicts the fact that $|\bar{q}(x)| \leq g$ a.e. in $\Omega$.

Moreover, from (2.16), we have that

$$
g(\sigma \bar{q}(x)+\nabla \bar{y}(x))=|\sigma \bar{q}(x)+\nabla \bar{y}(x)| \bar{q}(x),
$$

and it follows that

$$
g \nabla \bar{y}(x)=(|\sigma \bar{q}(x)+\nabla \bar{y}(x)|-\sigma g) \bar{q}(x) .
$$


Considering the norms in (2.18) and (2.19), we find that $(|\sigma \bar{q}(x)+\nabla \bar{y}(x)|-\sigma g)=|\nabla \bar{y}(x)|$ and thus we are in the second case of (2.15).

Reciprocally, assume that (2.9) holds and consider the two cases in (2.15). If $\nabla \bar{y}(x)=0$, we obtain that

$$
g(\sigma \bar{q}(x)+\nabla \bar{y}(x))=\max (\sigma g,|\sigma \bar{q}(x)+\nabla \bar{y}(x)|) \bar{q}(x)=\sigma g \bar{q}(x) .
$$

Similarly, if $\nabla \bar{y}(x) \neq 0$ and $\bar{q}(x)=g \frac{\nabla \bar{y}(x)}{|\nabla \bar{y}(x)|}$, we have that

$$
g(\sigma \bar{q}(x)+\nabla \bar{y}(x))=g \frac{\nabla \bar{y}(x)}{|\nabla \bar{y}(x)|}(\sigma g+|\nabla \bar{y}(x)|)
$$

which implies that

$$
\begin{aligned}
\max (\sigma g,|\sigma \bar{q}(x)+\nabla \bar{y}(x)|) \bar{q}(x) & =\max (\sigma g, \sigma g+|\nabla \bar{y}(x)|) g \frac{\nabla \bar{y}(x)}{|\nabla \bar{y}(x)|} \\
& =g \frac{\nabla \bar{y}(x)}{|\nabla \bar{y}(x)|}(\sigma g+|\nabla \bar{y}(x)|) .
\end{aligned}
$$

Thus, the equivalence follows.

Summarizing, we may rewrite (2.12) and (2.13) as the following system

$$
\begin{cases}a(\bar{y}, v)+(\bar{q}, \nabla v)_{\mathbf{L}^{2}(\Omega)}=(f, v)_{2}, & \text { for all } v \in H_{0}^{1}(\Omega), \\ \max (\sigma g,|\sigma \bar{q}(x)+\nabla \bar{y}(x)|) \bar{q}-g(\sigma \bar{q}+\nabla \bar{y})=0, & \text { a.e. in } \Omega \text { and for } \sigma>0 .\end{cases}
$$

We define the active and inactive sets for $(\mathcal{S})$ by $\mathcal{A}:=\{x \in \Omega:|\sigma \bar{q}(x)+\nabla \bar{y}(x)| \geq \sigma g\}$ and $\mathcal{I}:=\Omega \backslash \mathcal{A}$, respectively.

Remark 2.2. The solution to $(\mathcal{S})$ is not unique (see [12], Rem. 6.3, and [13], Chap. 5).

\subsection{Regularization}

In order to avoid problems related to the non-uniqueness of the solution to system $(\mathcal{S})$, we propose a Tikhonovtype regularization of $\left(\mathcal{P}^{*}\right)$. With this regularization procedure, we do not only achieve uniqueness of the solution but also get a local regularization for the non-differentiable term in $(\mathcal{P})$. This technique has also been used for TV-based inf-convolution-type image restoration [16].

For a parameter $\gamma>0$ we consider the following regularized dual problem

$$
\left\{\begin{array}{l}
\sup _{|q(x)| \leq g} J^{*}(q):=-\frac{1}{2} a(v(q), v(q))-\frac{1}{2 \gamma}\|q\|_{\mathbf{L}^{2}}^{2} \\
\text { where } v(q) \text { satisfies } \\
a(v(q), z)+(q, \nabla z)_{\mathbf{L}^{2}(\Omega)}-(f, z)_{2}=0, \text { for all } z \in H_{0}^{1}(\Omega) .
\end{array}\right.
$$

Therefore, the regularized problem is obtained from $\left(\mathcal{P}^{*}\right)$ by subtracting the term $\frac{1}{2 \gamma}\|q\|_{\mathbf{L}^{2}}^{2}$ from the objective functional. Further, it is possible to show that this penalization corresponds to a regularization of the primal problem. Consider the continuously differentiable function $\psi_{\gamma}: \mathbb{R}^{2} \rightarrow \mathbb{R}$, defined by

$$
\psi_{\gamma}(z):=\left\{\begin{array}{lll}
g|z|-\frac{g^{2}}{2 \gamma} & \text { if } & |z| \geq \frac{g}{\gamma} \\
\frac{\gamma}{2}|z|^{2} & \text { if } & |z|<\frac{g}{\gamma}
\end{array}\right.
$$


By using this function, which is a local regularization of the Euclidean norm, we obtain the following regularized version of $(\mathcal{P})$

$$
\min _{y \in H_{0}^{1}(\Omega)} J_{\gamma}(y):=\frac{1}{2} a(y, y)+\int_{\Omega} \psi_{\gamma}(\nabla y) \mathrm{d} x-(f, y)_{2} .
$$

Furthermore, we are able to state the following theorem.

Theorem 2.3. Problem $\left(\mathcal{P}_{\gamma}^{*}\right)$ is the dual problem of $\left(\mathcal{P}_{\gamma}\right)$ and we have

$$
J_{\gamma}^{*}\left(q_{\gamma}\right)=J_{\gamma}\left(y_{\gamma}\right)
$$

where $q_{\gamma}$ and $y_{\gamma}$ denote the solutions to $\left(\mathcal{P}_{\gamma}^{*}\right)$ and $\left(\mathcal{P}_{\gamma}\right)$, respectively.

Proof. In order to calculate the dual problem to $\left(\mathcal{P}_{\gamma}\right)$ we use the same argumentation used in Section 2.1 for the original problem $(\mathcal{P})$. We only have to replace the functional $\mathcal{G}$ in $(2.2)$ by

$$
\mathcal{G}_{\gamma}(q)=\int_{\Omega} \psi_{\gamma}(q) \mathrm{d} x
$$

with $\psi_{\gamma}$ as in (2.20). Thus, for $q \in \mathbf{L}^{2}(\Omega)$, we have

$$
\begin{aligned}
\mathcal{G}_{\gamma}^{*}(q)= & \sup _{p \in \mathbf{L}^{2}(\Omega)}\left\{\int_{\{x \in \Omega: \gamma|p(x)| \geq g, \text { a.e. }\}}\left[\langle q(x), p(x)\rangle-g|p(x)|+\frac{g^{2}}{2 \gamma}\right] \mathrm{d} x\right. \\
& \left.+\int_{\{x \in \Omega: \gamma|p(x)|<g, \text { a.e. }\}}\left[\langle q(x), p(x)\rangle-\frac{\gamma}{2}|p(x)|^{2}\right] \mathrm{d} x\right\} .
\end{aligned}
$$

From (2.23) it is possible to conclude, by proceeding as in the proof of Lemma 2.1, that $\mathcal{G}_{\gamma}(q)=\infty$ unless $|q(x)| \leq g$.

Suppose now that $|q(x)| \leq g$. We define the functional $\Psi: \mathbf{L}^{2}(\Omega) \rightarrow \mathbb{R}$ by

$$
\begin{aligned}
\Psi(p):= & \int_{\{x \in \Omega: \gamma|p(x)| \geq g, \text { a.e }\}}\left[\langle q(x), p(x)\rangle-g|p(x)|+\frac{g^{2}}{2 \gamma}\right] \mathrm{d} x \\
& +\int_{\{x \in \Omega: \gamma|p(x)|<g, \text { a.e }\}}\left[\langle q(x), p(x)\rangle-\frac{\gamma}{2}|p(x)|^{2}\right] \mathrm{d} x .
\end{aligned}
$$

By introducing, for any $p_{0} \in \mathbf{L}^{2}(\Omega)$, the function $\tilde{p}_{0} \in \mathbf{L}^{2}(\Omega)$ by

$$
\tilde{p}_{0}(x):=\left\{\begin{array}{lll}
p_{0}(x) & \text { a.e. in } & \{x \in \Omega: \gamma|p(x)|<g, \text { a.e. }\} \\
0 & \text { a.e. in } \quad\{x \in \Omega: \gamma|p(x)| \geq g, \text { a.e. }\}
\end{array}\right.
$$

it is easy to verify that $\Psi\left(p_{0}\right) \leq \Psi\left(\tilde{p}_{0}\right)$, which yields

$$
\sup _{p \in \mathbf{L}^{2}(\Omega)} \Psi(p)=\sup _{\substack{p \in \mathbf{L}^{2}(\Omega) \\ \gamma|p(x)| \leq g \text { a.e. in } \Omega}} \Psi(p) .
$$

Therefore, in order to calculate the supremum in (2.23), we only have to consider the last term in (2.24). Since this expression is a concave quadratical functional, the maximizer is easily calculated as $p_{0}=\gamma^{-1} p$, which 
implies that

$$
\mathcal{G}_{\gamma}^{*}(q)= \begin{cases}\frac{1}{2 \gamma}\|q\|_{\mathbf{L}^{2}}^{2} & \text { if }|q(x)| \leq g \\ \infty & \text { otherwise. }\end{cases}
$$

Note that this regularization procedure turns the primal problem into the unconstrained minimization of a continuously differentiable functional, while the corresponding dual problem is still the constrained minimization of a quadratic functional.

Remark 2.3. Due to the regularization procedure, the objective functional of $\left(\mathcal{P}_{\gamma}^{*}\right)$ results in a $\mathbf{L}^{2}(\Omega)$-uniformly concave functional. Thus, $\left(\mathcal{P}_{\gamma}^{*}\right)$ admits a unique solution $q_{\gamma} \in \mathbf{L}^{2}(\Omega)$ for each fixed $\gamma>0$. Additionally, since $a(\cdot, \cdot)$ is a coercive and bicontinuous form and due to the fact that $J_{\gamma}$ is strictly convex and differentiable, [21], Theorem 1.6, implies that $\left(\mathcal{P}_{\gamma}\right)$ has also a unique solution.

Theorem 2.4. Let $y_{\gamma}$ be the solution to $\left(\mathcal{P}_{\gamma}\right)$. Then $y_{\gamma} \in H^{2}(\Omega) \cap H_{0}^{1}(\Omega)$ and there exists a constant $K>0$, independent of $\gamma$, such that

for some $C>0$.

$$
\left\|y_{\gamma}\right\|_{H^{2}} \leq K\left(\|f\|_{L^{2}}+\sqrt{C}\right)
$$

Proof. Note that $y_{\gamma}$ can be characterized as the solution of the following equation

$$
\begin{array}{rll}
f \in-\mu \Delta y_{\gamma}+\partial \varphi\left(y_{\gamma}\right) & \text { in } & \Omega \\
y_{\gamma}=0 & \text { on } & \Gamma
\end{array}
$$

where $\partial \varphi$ denotes the subdifferential of the convex and lower semicontinuous functional $\varphi: L^{2}(\Omega) \rightarrow \mathbb{R} \cup\{\infty\}$ defined by

$$
\varphi(u)=\left\{\begin{array}{lc}
\int_{\Omega} \psi_{\gamma}(\nabla u) \mathrm{d} x & \text { if } \psi_{\gamma}(\nabla u) \in L^{1}(\Omega) \\
+\infty & \text { elsewhere }
\end{array}\right.
$$

Thus, [4], Lemma 1, implies the result.

Remark 2.4. Theorem 2.4 implies that $\nabla y_{\gamma} \in \mathbf{H}^{1}(\Omega)$ and, since $n=2, \nabla y_{\gamma} \in \mathbf{L}^{q}(\Omega)$, for all $q \in[1, \infty)$. Moreover, from (2.26) we conclude that $\nabla y_{\gamma}$ is uniformly bounded in $\mathbf{L}^{q}(\Omega)$ for all $q \in[1, \infty)$.

Next, we characterize the solutions to $\left(\mathcal{P}_{\gamma}\right)$ and $\left(\mathcal{P}_{\gamma}^{*}\right)\left(y_{\gamma}\right.$ and $q_{\gamma}$, respectively). From Fenchel's duality theory, these solutions satisfy the following system:

$$
\begin{aligned}
-\Lambda^{*} q_{\gamma} & \in \partial \mathcal{F}\left(y_{\gamma}\right) \\
q_{\gamma} & \in \partial \mathcal{G}_{\gamma}\left(\nabla y_{\gamma}\right) .
\end{aligned}
$$

Note that both $\mathcal{F}$ and $\mathcal{G}_{\gamma}$ are differentiable in $y_{\gamma}$ and $\nabla y_{\gamma}$, respectively. Thus, $\partial \mathcal{F}\left(y_{\gamma}\right)$ and $\partial \mathcal{G}_{\gamma}\left(\nabla y_{\gamma}\right)$ consist only of the respective Gateaux derivatives.

Since (2.28) is similar to equation (2.12), it is equivalent to

$$
a\left(y_{\gamma}, v\right)+\left(q_{\gamma}, \nabla v\right)_{\mathbf{L}^{2}(\Omega)}-(f, v)_{2}=0, \text { for all } v \in H_{0}^{1}(\Omega) .
$$

On the other hand, due to the differentiability of $\mathcal{G}_{\gamma}$, equation $(2.29)$ can be written as

$$
\left(q_{\gamma}, p\right)_{\mathbf{L}^{2}(\Omega)}=\int_{\mathcal{A}_{\gamma}} g \frac{\left\langle\nabla y_{\gamma}, p\right\rangle}{\left|\nabla y_{\gamma}\right|} \mathrm{d} x+\int_{\Omega \backslash \mathcal{A}_{\gamma}}\left\langle\gamma \nabla y_{\gamma}, p\right\rangle \mathrm{d} x, \text { for all } p \in \mathbf{L}^{2}(\Omega)
$$

or equivalently as

$$
\left\{\begin{array}{lll}
q_{\gamma}(x)=\gamma \nabla y_{\gamma}(x) & \text { a.e. in } & \Omega \backslash \mathcal{A}_{\gamma} \\
q_{\gamma}(x)=g \frac{\nabla y_{\gamma}(x)}{\left|\nabla y_{\gamma}\right|} & \text { a.e. in } & \mathcal{A}_{\gamma}
\end{array}\right.
$$


where, $\mathcal{A}_{\gamma}=\left\{x \in \Omega: \gamma\left|\nabla y_{\gamma}(x)\right| \geq g\right.$, a.e. $\}$. Consequently, the solutions $\left(y_{\gamma}, q_{\gamma}\right)$ of the regularized problems $\left(\mathcal{P}_{\gamma}\right)$ and $\left(\mathcal{P}_{\gamma}^{*}\right)$ satisfy the system

$$
\begin{cases}a\left(y_{\gamma}, v\right)+\left(q_{\gamma}, \nabla v\right)_{\mathbf{L}^{2}(\Omega)}-(f, v)_{2}=0, & \text { for all } v \in H_{0}^{1}(\Omega) \\ \max \left(g, \gamma\left|\nabla y_{\gamma}(x)\right|\right) q_{\gamma}(x)-g \gamma \nabla y_{\gamma}(x)=0, & \text { a.e. in } \Omega, \text { for all } \gamma>0 .\end{cases}
$$

Clearly $\left|q_{\gamma}(x)\right|=g$ on $\mathcal{A}_{\gamma}$ and $\left|q_{\gamma}(x)\right|<g$ on $\mathcal{I}_{\gamma}:=\Omega \backslash \mathcal{A}_{\gamma}$. We call sets $\mathcal{A}_{\gamma}$ and $\mathcal{I}_{\gamma}$ the active and inactive sets for $\left(\mathcal{S}_{\gamma}\right)$, respectively.

In the following theorem the convergence of the regularized solutions towards the original one is verified.

Theorem 2.5. The solutions $y_{\gamma}$ of the regularized primal problems converge to the solution $\bar{y}$ of the original problem strongly in $H_{0}^{1}(\Omega)$ as $\gamma \rightarrow \infty$. Moreover, the solutions $q_{\gamma}$ of the regularized dual problems converge to a solution $\bar{q}$ of the original dual problem weakly in $\mathbf{L}^{2}(\Omega)$.

Proof. Let us start by recalling that $(\bar{y}, \bar{q})$ and $\left(y_{\gamma}, q_{\gamma}\right)$ satisfy equations (2.14) and (2.30) respectively. Thus, by subtracting (2.30) from (2.14), we obtain that

$$
\mu \int_{\Omega}\left\langle\nabla\left(\bar{y}-y_{\gamma}\right), \nabla v\right\rangle \mathrm{d} x=\int_{\Omega}\left\langle q_{\gamma}-\bar{q}, \nabla v\right\rangle \mathrm{d} x, \text { for all } v \in H_{0}^{1}(\Omega) .
$$

Further, choosing $v:=\bar{y}-y_{\gamma}$ in (2.32), we have that

$$
\mu \int_{\Omega}\left|\nabla\left(\bar{y}-y_{\gamma}\right)\right|^{2} \mathrm{~d} x=\int_{\Omega}\left\langle q_{\gamma}-\bar{q}, \nabla\left(\bar{y}-y_{\gamma}\right)\right\rangle \mathrm{d} x .
$$

Next, we establish pointwise bounds for $\left\langle\left(q_{\gamma}-\bar{q}\right)(x), \nabla\left(\bar{y}-y_{\gamma}\right)(x)\right\rangle$, in the following disjoint sets $\mathcal{A} \cap \mathcal{A}_{\gamma}, \mathcal{A} \cap \mathcal{I}_{\gamma}$, $\mathcal{A}_{\gamma} \cap \mathcal{I}$ and $\mathcal{I}_{\gamma} \cap \mathcal{I}$.

On $\mathcal{A} \cap \mathcal{A}_{\gamma}$ : Here, we use the facts that $|\bar{q}(x)|=\left|q_{\gamma}(x)\right|=g, \bar{q}(x)=g \frac{\nabla \bar{y}(x)}{|\nabla \bar{y}(x)|}$ and $q_{\gamma}(x)=g \frac{\nabla y_{\gamma}(x)}{\left|\nabla y_{\gamma}(x)\right|}$. Thus, we have the following pointwise estimate

$$
\begin{aligned}
\left\langle\left(q_{\gamma}-\bar{q}\right)(x), \nabla\left(\bar{y}-y_{\gamma}\right)(x)\right\rangle \leq \leq & \left|q_{\gamma}(x)\right||\nabla \bar{y}(x)|-\left\langle g \frac{\nabla y_{\gamma}(x)}{\left|\nabla y_{\gamma}(x)\right|}, \nabla y_{\gamma}(x)\right\rangle \\
& -\left\langle g \frac{\nabla \bar{y}(x)}{|\nabla \bar{y}(x)|}, \nabla \bar{y}(x)\right\rangle+|\bar{q}(x)|\left|\nabla y_{\gamma}(x)\right| \\
\leq & g|\nabla \bar{y}(x)|-g\left|\nabla y_{\gamma}(x)\right|-g|\nabla \bar{y}(x)|+g\left|\nabla y_{\gamma}(x)\right|=0 .
\end{aligned}
$$

On $\mathcal{A} \cap \mathcal{I}_{\gamma}$ : Here, we know that $\nabla y_{\gamma}(x)=\gamma^{-1} q_{\gamma}(x),\left|q_{\gamma}(x)\right|<g,|\bar{q}(x)|=g$ and $\bar{q}(x)=g \frac{\nabla \bar{y}(x)}{|\nabla \bar{y}(x)|}$. Hence, we get

$$
\begin{aligned}
\left\langle\left(q_{\gamma}-\bar{q}\right)(x), \nabla\left(\bar{y}-y_{\gamma}\right)(x)\right\rangle & \leq g|\nabla \bar{y}(x)|-\gamma^{-1}\left|q_{\gamma}(x)\right|^{2}-g|\nabla \bar{y}(x)|+g\left|\nabla y_{\gamma}(x)\right| \\
& =-\gamma^{-1}\left|q_{\gamma}(x)\right|^{2}+g \gamma^{-1}\left|q_{\gamma}(x)\right| \\
& <\gamma^{-1}\left(g^{2}-\left|q_{\gamma}(x)\right|^{2}\right)<\gamma^{-1} g^{2} .
\end{aligned}
$$

On $\mathcal{A}_{\gamma} \cap \mathcal{I}$ : In this set it holds that $\nabla \bar{y}(x)=0$ and $q_{\gamma}(x)=g \frac{\nabla y_{\gamma}(x)}{\left|\nabla y_{\gamma}(x)\right|}$. Then, we have that

$$
\left\langle\left(q_{\gamma}-\bar{q}\right)(x), \nabla\left(\bar{y}-y_{\gamma}\right)(x)\right\rangle=-g\left|\nabla y_{\gamma}(x)\right|+|\bar{q}(x)|\left|\nabla y_{\gamma}(x)\right| \leq 0
$$

On $\mathcal{I}_{\gamma} \cap \mathcal{I}$ : Here, we have that $\nabla \bar{y}(x)=0, \nabla y_{\gamma}(x)=\gamma^{-1} q_{\gamma}(x),|\bar{q}(x)| \leq g$, and $\left|q_{\gamma}(x)\right|<g$.

$$
\begin{aligned}
\left\langle\left(q_{\gamma}-\bar{q}\right)(x), \nabla\left(\bar{y}-y_{\gamma}\right)(x)\right\rangle & =-\gamma^{-1}\left|q_{\gamma}(x)\right|^{2}+|\bar{q}(x)|\left|\nabla y_{\gamma}(x)\right| \\
& \leq \gamma^{-1}\left(g^{2}-\left|q_{\gamma}(x)\right|^{2}\right)<\gamma^{-1} g^{2} .
\end{aligned}
$$


Since $\mathcal{A} \cap \mathcal{A}_{\gamma}, \mathcal{A} \cap \mathcal{I}_{\gamma}, \mathcal{A}_{\gamma} \cap \mathcal{I}$ and $\mathcal{I}_{\gamma} \cap \mathcal{I}$ provide a disjoint partitioning of $\Omega$, (2.33) and estimates (2.34), (2.35), (2.36) and (2.37) imply that

$$
\mu \int_{\Omega}\left|\nabla\left(\bar{y}-y_{\gamma}\right)\right|^{2} \mathrm{~d} x<\int_{\Omega} \gamma^{-1} g^{2} \mathrm{~d} x .
$$

Thus, we conclude that $y_{\gamma} \rightarrow \bar{y}$ strongly in $H_{0}^{1}(\Omega)$ as $\gamma \rightarrow \infty$.

On the other hand, since $y_{\gamma} \rightarrow \bar{y}$ strongly in $H_{0}^{1}(\Omega),(2.32)$ implies that

$$
q_{\gamma} \rightarrow \bar{q}, \text { weakly in } \operatorname{grad}\left(H_{0}^{1}(\Omega)\right) \subset \mathbf{L}^{2}(\Omega),
$$

where $\operatorname{grad}\left(H_{0}^{1}(\Omega)\right):=\left\{q \in \mathbf{L}^{2}(\Omega): \exists v \in H_{0}^{1}(\Omega)\right.$ such that $\left.q=\nabla v\right\}$.

\section{PAth-Following Method}

In this section, we investigate the application of continuation strategies to properly control the increase of $\gamma$. Our main objective is to develop an automatic updating strategy for the regularization parameter which guarantees an efficient and fast approximation of the solution to problem $(\mathcal{P})$. For that purpose, we investigate the properties of the path $\gamma \mapsto\left(y_{\gamma}, q_{\gamma}\right) \in H_{0}^{1}(\Omega) \times \mathbf{L}^{2}(\Omega)$, with $\gamma \in(0, \infty)$, and construct an appropriate model of the value functional, which will be used in an updating algorithm.

\subsection{The primal-dual path}

In this part we introduce the primal-dual path and discuss some of its properties. Specifically, Lipschitz continuity and differentiability of the path are obtained.

Definition 3.1. The family of solutions $\mathcal{C}=\left\{\left(y_{\gamma}, q_{\gamma}\right): \gamma \in[M, \infty)\right\}$ to $\left(\mathcal{S}_{\gamma}\right)$, with $M$ a positive constant, considered as subset of $H_{0}^{1}(\Omega) \times \mathbf{L}^{2}(\Omega)$, is called the primal-dual path associated to $\left(\mathcal{P}_{\gamma}\right)-\left(\mathcal{P}_{\gamma}^{*}\right)$.

Lemma 3.1. The path $\mathcal{C}$ is bounded in $H_{0}^{1}(\Omega) \times \boldsymbol{L}^{2}(\Omega)$, i.e., there exist $C>0$, independent of $\gamma$, such that

$$
\left\|y_{\gamma}\right\|_{H_{0}^{1}}+\left\|q_{\gamma}\right\|_{L^{2}} \leq C
$$

Proof. First, from the fact that $\left|q_{\gamma}(x)\right| \leq g$, for every $\gamma>0$, we conclude that $q_{\gamma}$ is uniformly bounded in $\mathbf{L}^{2}(\Omega)$. Furthermore, Theorem 2.4 implies that $\left\|y_{\gamma}\right\|_{H_{0}^{1}}$ is uniformly bounded in $H_{0}^{1}(\Omega)$. Therefore, $\mathcal{C}$ is bounded in $H_{0}^{1}(\Omega) \times \mathbf{L}^{2}(\Omega)$.

Theorem 3.2. Let $\gamma \in[M, \infty)$. The function $\gamma \mapsto y_{\gamma}$ is globally Lipschitz continuous in $W_{0}^{1, p}(\Omega)$, for $2 \leq p<$ $2+\min (s-2, \epsilon)$, where $s>2$ and $\epsilon$ depends on $\mu, \gamma$ and $\Omega$.

Proof. Let $\gamma, \bar{\gamma} \in[M, \infty)$. We introduce the following notations $\delta_{y}:=y_{\bar{\gamma}}-y_{\gamma}, \theta_{\gamma}(x):=\max \left(g, \gamma\left|\nabla y_{\gamma}(x)\right|\right)$ and $\delta_{\theta}:=\theta_{\bar{\gamma}}-\theta_{\gamma}$. It is easy to verify that the following expression holds

$$
\left|\delta_{\theta}(x)\right| \leq|\bar{\gamma}| \nabla y_{\bar{\gamma}}(x)|-\gamma| \nabla y_{\gamma}(x)||, \text { a.e. in } \Omega,
$$

which implies that

$$
\left|\delta_{\theta}(x)\right| \leq|\gamma-\bar{\gamma}|\left|\nabla y_{\bar{\gamma}}(x)\right|+\gamma\left|\nabla \delta_{y}(x)\right|, \text { a.e. in } \Omega,
$$

and, similarly,

$$
\left|\delta_{\theta}(x)\right| \leq|\gamma-\bar{\gamma}|\left|\nabla y_{\gamma}(x)\right|+\bar{\gamma}\left|\nabla \delta_{y}(x)\right|, \text { a.e. in } \Omega .
$$

Next, we separate the proof in two parts. First, we prove the Lipschitz continuity of $\gamma \mapsto y_{\gamma}$ in $H_{0}^{1}(\Omega)$, and then, by introducing an auxiliar problem, we obtain the Lipschitz continuity in $W_{0}^{1, p}(\Omega)$, for some $p>2$. 
In $H_{0}^{1}(\Omega)$ : From $\left(\mathcal{S}_{\gamma}\right)$, we know that

$$
\begin{aligned}
a\left(\delta_{y}, \delta_{y}\right) & =g\left(\frac{\gamma \nabla y_{\gamma}}{\theta_{\gamma}}-\frac{\bar{\gamma} \nabla y_{\bar{\gamma}}}{\theta_{\bar{\gamma}}}, \nabla \delta_{y}\right)_{\mathbf{L}^{2}(\Omega)} \\
& =g(\gamma-\bar{\gamma})\left(\frac{\nabla y_{\bar{\gamma}}}{\theta_{\bar{\gamma}}}, \nabla \delta_{y}\right)_{\mathbf{L}^{2}(\Omega)}+g \gamma\left(\frac{\nabla y_{\gamma}}{\theta_{\gamma}}-\frac{\nabla y_{\bar{\gamma}}}{\theta_{\bar{\gamma}}}, \nabla \delta_{y}\right)_{\mathbf{L}^{2}(\Omega)}
\end{aligned}
$$

which, since $\frac{\left|\nabla y_{\bar{\gamma}}(x)\right|}{\theta_{\bar{\gamma}}} \leq \frac{1}{M}$, a.e. in $\Omega$, implies the existence of a constant $K>0$ such that

$$
a\left(\delta_{y}, \delta_{y}\right) \leq K|\gamma-\bar{\gamma}|\left\|\delta_{y}\right\|_{H_{0}^{1}}+g \gamma\left(\frac{\nabla y_{\gamma}}{\theta_{\gamma}}-\frac{\nabla y_{\bar{\gamma}}}{\theta_{\bar{\gamma}}}, \nabla \delta_{y}\right)_{\mathbf{L}^{2}(\Omega)}
$$

Next, let us analyze the second term on the right hand side of (3.4).

$$
\begin{aligned}
g \gamma\left(\frac{\nabla y_{\gamma}}{\theta_{\gamma}}-\frac{\nabla y_{\bar{\gamma}}}{\theta_{\bar{\gamma}}}, \nabla \delta_{y}\right)_{\mathbf{L}^{2}(\Omega)} & =g \gamma\left(\frac{-\theta_{\gamma} \nabla \delta_{y}+\delta_{\theta} \nabla y_{\gamma}}{\theta_{\gamma} \theta_{\bar{\gamma}}}, \nabla \delta_{y}\right)_{\mathbf{L}^{2}(\Omega)} \\
& =-g \gamma \int_{\Omega} \frac{\left|\nabla \delta_{y}(x)\right|^{2}}{\theta_{\bar{\gamma}}} \mathrm{d} x+g \gamma\left(\frac{\delta_{\theta} \nabla y_{\gamma}}{\theta_{\gamma} \theta_{\bar{\gamma}}}, \nabla \delta_{y}\right)_{\mathbf{L}^{2}(\Omega)}
\end{aligned}
$$

Since $\gamma\left|\nabla y_{\gamma}(x)\right| \leq \theta_{\gamma}(x)$ a.e. in $\Omega$, Cauchy-Schwarz inequality implies that

$$
\begin{aligned}
g \gamma\left|\left(\frac{\delta_{\theta} \nabla y_{\gamma}}{\theta_{\gamma} \theta_{\bar{\gamma}}}, \nabla \delta_{y}\right)_{\mathbf{L}^{2}(\Omega)}\right| & \leq g \gamma \int_{\Omega}\left|\left\langle\frac{\delta_{\theta} \nabla y_{\gamma}(x)}{\theta_{\gamma}(x) \theta_{\bar{\gamma}}(x)}, \nabla \delta_{y}(x)\right\rangle\right| \mathrm{d} x \\
& \leq g \int_{\Omega} \frac{\left|\delta_{\theta}(x)\right| \gamma\left|\nabla y_{\gamma}(x)\right|\left|\nabla \delta_{y}(x)\right|}{\theta_{\gamma}(x) \theta_{\bar{\gamma}}(x)} \mathrm{d} x \\
& \leq g \int_{\Omega} \frac{\left|\delta_{\theta}(x)\right|\left|\nabla \delta_{y}(x)\right|}{\theta_{\bar{\gamma}}(x)} \mathrm{d} x .
\end{aligned}
$$

Again, since $\bar{\gamma}\left|\nabla y_{\bar{\gamma}}(x)\right| \leq \theta_{\bar{\gamma}}(x)$ a.e. in $\Omega,(3.1)$ implies that

$$
\begin{aligned}
g \gamma\left|\left(\frac{\delta_{\theta} \nabla y_{\gamma}}{\theta_{\gamma} \theta_{\bar{\gamma}}}, \nabla \delta_{y}\right)_{\mathbf{L}^{2}(\Omega)}\right| & \leq g|\bar{\gamma}-\gamma| \int_{\Omega} \frac{\left|\nabla y_{\bar{\gamma}}\right|\left|\nabla \delta_{y}\right|}{\theta_{\bar{\gamma}}} \mathrm{d} x+g \gamma \int_{\Omega} \frac{\left|\nabla \delta_{y}\right|^{2}}{\theta_{\bar{\gamma}}} \mathrm{d} x \\
& \leq g \frac{|\bar{\gamma}-\gamma|}{\bar{\gamma}} \int_{\Omega}\left|\nabla \delta_{y}\right| \mathrm{d} x+g \gamma \int_{\Omega} \frac{\left|\nabla \delta_{y}\right|^{2}}{\theta_{\bar{\gamma}}} \mathrm{d} x \\
& \leq g \frac{|\bar{\gamma}-\gamma|}{M} \operatorname{meas}(\Omega)^{1 / 2}\left\|\delta_{y}\right\|_{H_{0}^{1}}+g \gamma \int_{\Omega} \frac{\left|\nabla \delta_{y}\right|^{2}}{\theta_{\bar{\gamma}}} \mathrm{d} x .
\end{aligned}
$$

Finally, using (3.6) and (3.5) in (3.4), we have that

$$
a\left(\delta_{y}, \delta_{y}\right) \leq\left(K+\frac{g \operatorname{meas}(\Omega)^{1 / 2}}{M}\right)|\bar{\gamma}-\gamma|\left\|\delta_{y}\right\|_{H_{0}^{1}},
$$

which, due to the coercivity of $a(\cdot, \cdot)$, implies the existence of a constant $L>0$ such that

$$
\left\|y_{\bar{\gamma}}-y_{\gamma}\right\|_{H_{0}^{1}} \leq L|\bar{\gamma}-\gamma|
$$


In $W_{0}^{1, p}(\Omega)$ : First, note that (3.2) implies the existence of $\zeta(x) \in[-1,1]$ such that

$$
\delta_{\theta}(x)=\zeta(x)\left[|\bar{\gamma}-\gamma|\left|\nabla y_{\gamma}(x)\right|+\bar{\gamma}|\nabla \delta y(x)|\right], \text { a.e. in } \Omega .
$$

From $\left(\mathcal{S}_{\gamma}\right)$, we have, for all $v \in H_{0}^{1}(\Omega)$, that

$$
a\left(\delta_{y}, v\right)+g \bar{\gamma}\left(\frac{\nabla \delta_{y}}{\theta_{\gamma}}, \nabla v\right)_{\mathbf{L}^{2}(\Omega)}-g \bar{\gamma}\left(\frac{\delta_{\theta} \nabla y_{\bar{\gamma}}}{\theta_{\gamma} \theta_{\bar{\gamma}}}, \nabla v\right)_{\mathbf{L}^{2}(\Omega)}=g(\gamma-\bar{\gamma})\left(\frac{\nabla y_{\gamma}}{\theta_{\gamma}}, \nabla v\right)_{\mathbf{L}^{2}(\Omega)},
$$

which, together with (3.7), implies that

$$
\begin{aligned}
a\left(\delta_{y}, v\right)+g \bar{\gamma}\left(\frac{\nabla \delta_{y}}{\theta_{\gamma}}, \nabla v\right)_{\mathbf{L}^{2}(\Omega)}-g \bar{\gamma}\left(\frac{\zeta(x)\left|\nabla \delta_{y}\right|}{\theta_{\bar{\gamma}} \theta_{\gamma}} \bar{\gamma} \nabla y_{\bar{\gamma}}, \nabla v\right)_{\mathbf{L}^{2}(\Omega)}= & \\
& g(\gamma-\bar{\gamma})\left(\frac{\nabla y_{\gamma}}{\theta_{\gamma}}, \nabla v\right)_{\mathbf{L}^{2}(\Omega)}+g|\gamma-\bar{\gamma}|\left(\frac{\zeta(x)\left|\nabla y_{\gamma}\right|}{\theta_{\bar{\gamma}} \theta_{\gamma}} \bar{\gamma} \nabla y_{\bar{\gamma}}, \nabla v\right)_{\mathbf{L}^{2}(\Omega)},
\end{aligned}
$$

for all $v \in H_{0}^{1}(\Omega)$. Defining $\overline{\mathbf{f}}:=g(\gamma-\bar{\gamma}) \frac{\nabla y_{\gamma}}{\theta_{\gamma}}+g|\gamma-\bar{\gamma}| \frac{\zeta(x)\left|\nabla y_{\gamma}\right|}{\theta_{\bar{\gamma}} \theta_{\gamma}} \bar{\gamma} \nabla y_{\bar{\gamma}}$, equation (3.9) motivates the introduction of the following auxiliar problem: find $w \in H_{0}^{1}(\Omega)$ such that

$$
a(w, v)+(\beta(w), \nabla v)_{\mathbf{L}^{2}(\Omega)}=(\overline{\mathbf{f}}, \nabla v)_{\mathbf{L}^{2}(\Omega)}, \text { for all } v \in H_{0}^{1}(\Omega),
$$

where $\beta(w):=g \bar{\gamma}\left[\frac{\nabla w}{\theta_{\gamma}}-\frac{\zeta(x)\left\langle\nabla \delta_{y}, \nabla w\right\rangle}{\theta_{\bar{\gamma}} \theta_{\gamma}\left|\nabla \delta_{y}\right|} \bar{\gamma} \nabla y_{\bar{\gamma}}\right]$. Clearly, $\delta_{y}$ is also solution of (3.10).

Note that

$$
|\overline{\mathbf{f}}(x)| \leq g|\gamma-\bar{\gamma}|\left|\frac{\nabla y_{\gamma}(x)}{\theta_{\gamma}(x)}\right|+g|\gamma-\bar{\gamma}| \frac{\zeta(x)\left|\nabla y_{\gamma}(x)\right|}{\theta_{\bar{\gamma}}(x) \theta_{\gamma}(x)} \bar{\gamma} \nabla y_{\bar{\gamma}}(x) \mid, \text { a.e. in } \Omega,
$$

which, since $\frac{\left|\nabla y_{\gamma}(x)\right|}{\theta_{\gamma}} \leq \frac{1}{M}$ and $\bar{\gamma}\left|\nabla y_{\bar{\gamma}}(x)\right| \leq \theta_{\bar{\gamma}}$, a.e. in $\Omega$, implies that

$$
|\overline{\mathbf{f}}(x)| \leq \frac{2 g}{M}|\gamma-\bar{\gamma}| \text { a.e. in } \Omega .
$$

Therefore,

$$
\|\overline{\mathbf{f}}\|_{\mathbf{L}^{s}} \leq \frac{2 g}{M} \operatorname{meas}(\Omega)^{1 / s}|\bar{\gamma}-\gamma|, \text { for } 1 \leq s \leq \infty .
$$

Next, let us define the matrix $A(x) \in \mathbb{R}^{2 \times 2}$ by

$$
A(x):=\bar{\gamma}\left(\begin{array}{ll}
\frac{\partial y_{\bar{\gamma}}(x)}{\partial x_{1}} \frac{\partial \delta_{y}(x)}{\partial x_{1}} & \frac{\partial y_{\bar{\gamma}}(x)}{\partial x_{1}} \frac{\partial \delta_{y}(x)}{\partial x_{2}} \\
\frac{\partial y_{\bar{\gamma}}(x)}{\partial x_{2}} \frac{\partial \delta_{y}(x)}{\partial x_{1}} & \frac{\partial y_{\bar{\gamma}}(x)}{\partial x_{2}} \frac{\partial \delta_{y}(x)}{\partial x_{2}}
\end{array}\right), \text { a.e. in } \Omega .
$$

Then, we can rewrite $\beta(w)$ as

$$
\beta(w)=g \bar{\gamma}\left[\frac{I}{\theta_{\gamma}}-\frac{\zeta(x)}{\theta_{\gamma} \theta_{\bar{\gamma}}\left|\nabla \delta_{y}\right|} A(x)\right] \nabla w,
$$

where $I$ stands for the $2 \times 2$-identity matrix. Moreover, we can rewrite the auxiliar problem (3.10) as

$$
\int_{\Omega} \alpha(x)\langle\nabla w, \nabla v\rangle \mathrm{d} x=\int_{\Omega}\langle\overline{\mathbf{f}}, \nabla v\rangle \mathrm{d} x,
$$


where $\alpha(x):=\left(\mu+\frac{g \bar{\gamma}}{\theta_{\gamma}}(x)\right) I+g \bar{\gamma} \frac{\zeta(x)}{\theta_{\gamma}(x) \theta_{\bar{\gamma}}(x)\left|\nabla \delta_{y}(x)\right|} A(x)$, a.e. in $\Omega$. Multiplying $\alpha(x)$ by $\xi \in \mathbb{R}^{2 \times 2}$ and taking the scalar product with $\xi$, we obtain that

$$
\langle\alpha(x) \xi, \xi\rangle=\mu|\xi|^{2}+\frac{g \bar{\gamma}}{\theta_{\gamma}(x)}|\xi|^{2}-g \bar{\gamma} \frac{\zeta(x)}{\theta_{\gamma}(x) \theta_{\bar{\gamma}}(x)\left|\nabla \delta_{y}(x)\right|}\left\langle\nabla \delta_{y}(x), \xi\right\rangle\left\langle\bar{\gamma} \nabla y_{\bar{\gamma}}(x), \xi\right\rangle,
$$

a.e. in $\Omega$. Furthermore, since $|\zeta(x)| \leq 1$ and $\bar{\gamma}\left|\nabla y_{\bar{\gamma}}(x)\right| \leq \theta_{\bar{\gamma}}(x)$ a.e. in $\Omega$, the Cauchy-Schwarz inequality implies that

$$
g \bar{\gamma}\left|\frac{\zeta(x)}{\theta_{\gamma}(x) \theta_{\bar{\gamma}}(x)\left|\nabla \delta_{y}(x)\right|}\left\langle\nabla \delta_{y}(x), \xi\right\rangle\left\langle\bar{\gamma} \nabla y_{\bar{\gamma}}(x), \xi\right\rangle\right| \leq g \bar{\gamma} \frac{|\xi|^{2}}{\theta_{\gamma}(x)}, \text { a.e. in } \Omega,
$$

which, due to the fact that $g \leq \theta_{\gamma}(x)$, a.e. in $\Omega$, implies that

$$
\mu|\xi|^{2} \leq\langle\alpha(x) \xi, \xi\rangle \leq(\mu+2 \bar{\gamma})|\xi|^{2} \text {, a.e. in } \Omega .
$$

Thus, [3], Theorem 2.1, p. 64, implies the existence of a constant $c_{p}$ such that

$$
\|\bar{w}\|_{W_{0}^{1, p}} \leq c_{p}\|\overline{\mathbf{f}}\|_{L^{s}}, \text { for } s>2 \text { and } 2 \leq p<2+\min (s-2, \epsilon),
$$

where $\bar{w}$ is the unique solution of (3.10) and $\epsilon$ depends on $\mu, \bar{\gamma}$ and $\Omega$. Therefore, since $\delta_{y}$ is solution of (3.10), estimates (3.11) and (3.15) imply the existence of $L_{1}>0$ such that

$$
\left\|y_{\bar{\gamma}}-y_{\gamma}\right\|_{W_{0}^{1, p}} \leq L_{1}|\bar{\gamma}-\gamma| \text {, for } 2 \leq p<2+\min (s-2, \epsilon) .
$$

Remark 3.2. Since $\gamma \mapsto y_{\gamma}$ is Lipschitz continuous in $W_{0}^{1, p}(\Omega)$, for some $p>2$, there exists a weak accumulation point $\dot{y}_{\gamma} \in W_{0}^{1, p}(\Omega)$ of $\frac{1}{\bar{\gamma}-\gamma}\left(y_{\bar{\gamma}}-y_{\gamma}\right)$ as $\bar{\gamma} \rightarrow \gamma$, which is a strong accumulation point in $H_{0}^{1}(\Omega)$.

For the subsequent analysis and the remaining sections of the paper, we will use the following assumption.

Assumption 3.3. Let $\gamma \in[M, \infty)$. There exist $\varepsilon_{1}, \varepsilon_{2}>0$ and $r>0$ such that

$$
\begin{aligned}
& \operatorname{meas}\left(\left\{x \in \mathcal{A}_{\bar{\gamma}} \cap \mathcal{I}_{\gamma}: \bar{\gamma}\left|\nabla y_{\bar{\gamma}}(x)\right|-\gamma\left|\nabla y_{\gamma}(x)\right|<\varepsilon_{1}\right\}\right)=0, \\
& \operatorname{meas}\left(\left\{x \in \mathcal{A}_{\gamma} \cap \mathcal{I}_{\bar{\gamma}}: \gamma\left|\nabla y_{\gamma}(x)\right|-\bar{\gamma}\left|\nabla y_{\bar{\gamma}}(x)\right|<\varepsilon_{2}\right\}\right)=0,
\end{aligned}
$$

for all $\bar{\gamma} \in(\gamma-r, \gamma+r)$.

Lemma 3.4. Let $\gamma \in[M, \infty)$ be fixed, and let $\bar{\gamma} \in(\gamma-r, \gamma+r)$. It holds that

$$
\lim _{\bar{\gamma} \rightarrow \gamma} \operatorname{meas}\left(\mathcal{A}_{\bar{\gamma}} \cap \mathcal{I}_{\gamma}\right)=\lim _{\bar{\gamma} \rightarrow \gamma} \operatorname{meas}\left(\mathcal{A}_{\gamma} \cap \mathcal{I}_{\bar{\gamma}}\right)=0 .
$$

Proof. Let us introduce the set $A_{\varepsilon_{1}}:=\left\{x \in \mathcal{A}_{\bar{\gamma}} \cap \mathcal{I}_{\gamma}:|\bar{\gamma}| \nabla y_{\bar{\gamma}}|-\gamma| \nabla y_{\gamma}|| \geq \varepsilon_{1}\right\}$. From assumption (3.3), we get that

$$
\operatorname{meas}\left(\mathcal{A}_{\bar{\gamma}} \cap \mathcal{I}_{\gamma}\right) \leq \operatorname{meas}\left(A_{\varepsilon_{1}}\right) \text {. }
$$

Due to Chebyshev's inequality we get that

$$
\begin{aligned}
\varepsilon_{1} \operatorname{meas}\left(A_{\varepsilon_{1}}\right) & \leq \int_{\mathcal{A}_{\bar{\gamma}} \cap \mathcal{I}_{\gamma}}|\bar{\gamma}| \nabla y_{\bar{\gamma}}(x)|-\gamma| \nabla y_{\gamma}(x)|| \mathrm{d} x \\
& \leq|\bar{\gamma}-\gamma| \int_{\Omega}\left|\nabla y_{\bar{\gamma}}(x)\right| \mathrm{d} x+\gamma \int_{\Omega}\left|\nabla y_{\bar{\gamma}}(x)-\nabla y_{\gamma}(x)\right| \mathrm{d} x
\end{aligned}
$$


which, by Lemma 3.1 and Theorem 3.2, implies that

$$
\begin{aligned}
\varepsilon_{1} \operatorname{meas}\left(A_{\varepsilon_{1}}\right) & \leq|\bar{\gamma}-\gamma|(\operatorname{meas}(\Omega))^{1 / 2}\left\|y_{\bar{\gamma}}\right\|_{H_{0}^{1}}+\gamma(\operatorname{meas}(\Omega))^{1 / 2}\left\|y_{\bar{\gamma}}-y_{\gamma}\right\|_{H_{0}^{1}} \\
& \leq K_{\Omega}|\bar{\gamma}-\gamma|,
\end{aligned}
$$

for some $K_{\Omega}>0$. Therefore,

$$
\lim _{\bar{\gamma} \rightarrow \gamma} \operatorname{meas}\left(\left\{x \in \Omega:|\bar{\gamma}| \nabla y_{\bar{\gamma}}(x)|-\gamma| \nabla y_{\gamma}(x)|| \geq \varepsilon_{1}\right\}\right)=0,
$$

and the result follows from (3.16). The other case is treated similarly.

As a consequence of Lemma 3.4 we also obtain that

$$
\lim _{\bar{\gamma} \rightarrow \gamma} \operatorname{meas}\left(\mathcal{A}_{\bar{\gamma}} \cap \mathcal{A}_{\gamma}\right)=\operatorname{meas}\left(\mathcal{A}_{\gamma}\right) \text { and } \lim _{\bar{\gamma} \rightarrow \gamma} \operatorname{meas}\left(\mathcal{I}_{\bar{\gamma}} \cap \mathcal{I}_{\gamma}\right)=\operatorname{meas}\left(\mathcal{I}_{\gamma}\right)
$$

which, since $\mathcal{A}_{\gamma}=\left(\mathcal{A}_{\gamma} \cap \mathcal{A}_{\bar{\gamma}}\right) \cup\left(\mathcal{A}_{\gamma} \backslash \mathcal{A}_{\bar{\gamma}}\right)$ and $\mathcal{I}_{\gamma}=\left(\mathcal{I}_{\gamma} \cap \mathcal{I}_{\bar{\gamma}}\right) \cup\left(\mathcal{I}_{\gamma} \backslash \mathcal{I}_{\bar{\gamma}}\right)$, implies that

$$
\lim _{\bar{\gamma} \rightarrow \gamma}\left(\mathcal{A}_{\gamma} \backslash \mathcal{A}_{\bar{\gamma}}\right)=\lim _{\bar{\gamma} \rightarrow \gamma}\left(\mathcal{I}_{\gamma} \backslash \mathcal{I}_{\bar{\gamma}}\right)=0
$$

Proposition 3.5. Let $\gamma>M$ and $\dot{y}_{\gamma}^{+}$be a weak accumulation point of $\frac{1}{\bar{\gamma}-\gamma}\left(y_{\bar{\gamma}}-y_{\gamma}\right)$ in $W_{0}^{1, p}(\Omega)$, for some $p>2$, as $\bar{\gamma} \downarrow \gamma$. Then $\dot{y}_{\gamma}^{+}$satisfies

$$
\begin{aligned}
a\left(\dot{y}_{\gamma}^{+}, v\right)+g\left(\left(\frac{\nabla \dot{y}_{\gamma}^{+}}{\left|\nabla y_{\gamma}\right|}-\frac{\left\langle\nabla y_{\gamma}, \nabla \dot{y}_{\gamma}^{+}\right\rangle}{\left|\nabla y_{\gamma}\right|^{3}} \nabla y_{\gamma}\right) \chi_{\mathcal{A}_{\gamma}}, \nabla v\right)_{L^{2}(\Omega)} \\
+\left(\left(\nabla y_{\gamma}+\gamma \nabla \dot{y}_{\gamma}^{+}\right) \chi_{\mathcal{I}_{\gamma}}, \nabla v\right)_{L^{2}(\Omega)}=0 .
\end{aligned}
$$

Proof. See the Appendix.

Proceeding as in Proposition 3.5, we also obtain that

$$
\begin{aligned}
a\left(\dot{y}_{\gamma}^{-}, v\right)+g\left(\left(\frac{\nabla \dot{y}_{\gamma}^{-}}{\left|\nabla y_{\gamma}\right|}\right.\right. & \left.\left.-\frac{\left\langle\nabla y_{\gamma}, \nabla \dot{y}_{\gamma}^{-}\right\rangle}{\left|\nabla y_{\gamma}\right|^{3}} \nabla y_{\gamma}\right) \chi_{\mathcal{A}_{\gamma}}, \nabla v\right)_{\mathbf{L}^{2}(\Omega)} \\
& +\left(\left(\nabla y_{\gamma}+\gamma \nabla \dot{y}_{\gamma}^{-}\right) \chi_{\mathcal{I}_{\gamma}}, \nabla v\right)_{\mathbf{L}^{2}(\Omega)}=0,
\end{aligned}
$$

where $\dot{y}_{\gamma}^{-}$stand for any weak accumulation point of $\frac{1}{\bar{\gamma}-\gamma}\left(y_{\bar{\gamma}}-y_{\gamma}\right)$ in $W_{0}^{1, p}(\Omega)$, for some $p>2$, as $\bar{\gamma} \uparrow \gamma$. Therefore, we obtain the following result.

Theorem 3.6. The function $\gamma \rightarrow y_{\gamma} \in H_{0}^{1}(\Omega)$ is differentiable at all $\gamma \in[M,+\infty)$, and $\dot{y}_{\gamma}$ satisfies

$$
\begin{aligned}
a\left(\dot{y}_{\gamma}, v\right)+g\left(\left(\frac{\nabla \dot{y}_{\gamma}}{\left|\nabla y_{\gamma}\right|}-\frac{\left\langle\nabla y_{\gamma}, \nabla \dot{y}_{\gamma}\right\rangle}{\left|\nabla y_{\gamma}\right|^{3}} \nabla y_{\gamma}\right) \chi_{\mathcal{A}_{\gamma}}, \nabla v\right)_{L^{2}(\Omega)} \\
+\left(\left(\nabla y_{\gamma}+\gamma \nabla \dot{y}_{\gamma}\right) \chi_{\mathcal{I}_{\gamma}}, \nabla v\right)_{L^{2}(\Omega)}=0 .
\end{aligned}
$$

Proof. Let $z$ denote the difference between two accumulation points of $(\bar{\gamma}-\gamma)^{-1}\left(y_{\bar{\gamma}}-y_{\gamma}\right)$ as $\bar{\gamma} \rightarrow \gamma$. From (3.19) and (3.20) we obtain that

$$
a(z, v)+g\left(\left(\frac{\nabla z}{\left|\nabla y_{\gamma}\right|}-\frac{\left\langle\nabla y_{\gamma}, \nabla z\right\rangle}{\left|\nabla y_{\gamma}\right|^{3}} \nabla y_{\gamma}\right) \chi_{\mathcal{A}_{\gamma}}+\gamma \nabla z \chi_{\mathcal{I}_{\gamma}}, \nabla v\right)_{\mathbf{L}^{2}(\Omega)}=0 .
$$


Choosing $v=z$ in the last expression, we obtain that

$$
\mu\|z\|_{H_{0}^{1}}^{2}+\gamma\|\nabla z\|_{\mathbf{L}^{2}\left(\mathcal{I}_{\gamma}\right)}^{2}+g\left(\frac{\nabla z}{\left|\nabla y_{\gamma}\right|}-\frac{\left\langle\nabla y_{\gamma}, \nabla z\right\rangle}{\left|\nabla y_{\gamma}\right|^{3}} \nabla y_{\gamma}, \nabla z\right)_{\mathbf{L}^{2}\left(\mathcal{A}_{\gamma}\right)}=0 .
$$

Since

$$
\left(\frac{\nabla z}{\left|\nabla y_{\gamma}\right|}-\frac{\left\langle\nabla y_{\gamma}, \nabla z\right\rangle}{\left|\nabla y_{\gamma}\right|^{3}} \nabla y_{\gamma}, \nabla z\right)_{\mathbf{L}^{2}\left(\mathcal{A}_{\gamma}\right)} \geq 0
$$

we get, from (3.22), that $z=0$. Consequently, accumulation points are unique and by (3.19) and (3.20) they satisfy (3.21).

\subsection{Path value functional}

In this section we study the value functional associated to $\left(\mathcal{P}_{\gamma}\right)$. We prove that the functional is twice differentiable with non positive second derivative, which implies concavity of the functional.

Definition 3.3. The functional

$$
\gamma \mapsto V(\gamma):=J_{\gamma}\left(y_{\gamma}\right)
$$

defined on $[M, \infty), M>0$, is called the path value functional.

Let us start by analyzing the differentiability properties of $V$.

Proposition 3.7. Let $\gamma \in[M, \infty)$. The value functional $V$ is differentiable at $\gamma$, with

$$
\dot{V}(\gamma)=\frac{1}{2 \gamma^{2}} \int_{\mathcal{A}_{\gamma}} g^{2} \mathrm{~d} x+\frac{1}{2} \int_{\mathcal{I}_{\gamma}}\left|\nabla y_{\gamma}\right|^{2} \mathrm{~d} x
$$

Proof. Let $r>0$ be sufficiently small and let $\bar{\gamma} \in(\gamma-r, \gamma+r)$. From (2.30) and by choosing $v=y_{\bar{\gamma}}-y_{\gamma}$, we find that

$$
\frac{1}{2} a\left(y_{\bar{\gamma}}+y_{\gamma}, y_{\bar{\gamma}}-y_{\gamma}\right)+\frac{1}{2}\left(q_{\bar{\gamma}}+q_{\gamma}, \nabla\left(y_{\bar{\gamma}}-y_{\gamma}\right)\right)_{\mathbf{L}^{2}(\Omega)}-\left(f, y_{\bar{\gamma}}-y_{\gamma}\right)_{2}=0 .
$$

On the other hand, note that

$$
\frac{1}{2} a\left(y_{\bar{\gamma}}+y_{\gamma}, y_{\bar{\gamma}}-y_{\gamma}\right)=\frac{1}{2} a\left(y_{\bar{\gamma}}, y_{\bar{\gamma}}\right)-\frac{1}{2} a\left(y_{\gamma}, y_{\gamma}\right)
$$

Consequently, from (3.24), we obtain that

$$
\begin{aligned}
V(\bar{\gamma})-V(\gamma) & =\frac{1}{2} a\left(y_{\bar{\gamma}}, y_{\bar{\gamma}}\right)-\frac{1}{2} a\left(y_{\gamma}, y_{\gamma}\right)-\left(f, y_{\bar{\gamma}}-y_{\gamma}\right)_{2}+\int_{\Omega}\left[\psi_{\bar{\gamma}}\left(\nabla y_{\bar{\gamma}}\right)-\psi_{\gamma}\left(\nabla y_{\gamma}\right)\right] \mathrm{d} x \\
& =\int_{\Omega}\left[\psi_{\bar{\gamma}}\left(\nabla y_{\bar{\gamma}}\right)-\psi_{\gamma}\left(\nabla y_{\gamma}\right)\right] \mathrm{d} x-\frac{1}{2}\left(q_{\bar{\gamma}}+q_{\gamma}, \nabla\left(y_{\bar{\gamma}}-y_{\gamma}\right)\right)_{\mathbf{L}^{2}(\Omega)},
\end{aligned}
$$

where $\psi_{\gamma}$ is defined by $(2.20)$. Then, from $\left(\mathcal{S}_{\gamma}\right)$, we conclude that

$$
V(\bar{\gamma})-V(\gamma)=\int_{\Omega} z \mathrm{~d} x
$$

where $z$ is defined by

$$
z(x):=\left[\psi_{\gamma}\left(\nabla y_{\bar{\gamma}}(x)\right)-\psi_{\gamma}\left(\nabla y_{\gamma}(x)\right)-\frac{g}{2}\left\langle\frac{\bar{\gamma} \nabla y_{\bar{\gamma}}(x)}{\theta_{\bar{\gamma}}(x)}+\frac{\gamma \nabla y_{\gamma}(x)}{\theta_{\gamma}(x)}, \nabla\left(y_{\bar{\gamma}}-y_{\gamma}\right)(x)\right\rangle\right]
$$




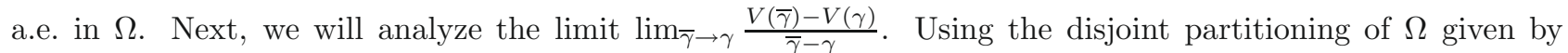
$\Omega_{1}:=\mathcal{A}_{\bar{\gamma}} \cap \mathcal{A}_{\gamma}, \Omega_{2}:=\mathcal{A}_{\bar{\gamma}} \cap \mathcal{I}_{\gamma}, \Omega_{3}:=\mathcal{A}_{\gamma} \cap \mathcal{I}_{\bar{\gamma}}$, and $\Omega_{4}:=\mathcal{I}_{\gamma} \cap \mathcal{I}_{\bar{\gamma}}$, we get that

$$
V(\bar{\gamma})-V(\gamma)=\sum_{j=1}^{4} \int_{\Omega_{j}} z_{j} \mathrm{~d} x,
$$

where $z_{j}$ represents the value of $z$ when restricted to each set $\Omega_{j}, j=1, \ldots, 4$. Now, we analyze each integral $\int_{\Omega_{j}} z_{j} \mathrm{~d} x$ separately.

On $\Omega_{1}$ : Here, we analyze the limit $\lim _{\bar{\gamma} \rightarrow \gamma} \frac{1}{\bar{\gamma}-\gamma} \int_{\Omega_{1}} z_{1} \mathrm{~d} x$. We start by recalling that a.e. in $\Omega_{1}$, we have that $\theta_{\bar{\gamma}}(x)=\bar{\gamma}\left|\nabla y_{\bar{\gamma}}(x)\right|, \theta_{\gamma}(x)=\gamma\left|\nabla y_{\gamma}(x)\right|, \psi\left(\nabla y_{\gamma}(x)\right)=g\left|\nabla y_{\gamma}(x)\right|-\frac{g^{2}}{2 \gamma}$ and $\psi\left(\nabla y_{\bar{\gamma}}(x)\right)=g\left|\nabla y_{\bar{\gamma}}(x)\right|-\frac{g^{2}}{2 \bar{\gamma}}$. Thus, we obtain the following pointwise a.e. estimate

$$
\begin{aligned}
z_{1}(x)= & g\left[\left|\nabla y_{\bar{\gamma}}(x)\right|-\left|\nabla y_{\gamma}(x)\right|\right]+\frac{g^{2}}{2}\left[\frac{\bar{\gamma}-\gamma}{\bar{\gamma} \gamma}\right] \\
& -\frac{g}{2}\left\langle\frac{\nabla y_{\bar{\gamma}}(x)}{\left|\nabla y_{\bar{\gamma}}(x)\right|}+\frac{\nabla y_{\gamma}(x)}{\left|\nabla y_{\gamma}(x)\right|}, \nabla\left(y_{\bar{\gamma}}(x)-y_{\gamma}(x)\right)\right\rangle \\
= & \frac{g}{2}\left[\left|\nabla y_{\bar{\gamma}}(x)\right|-\left|\nabla y_{\gamma}(x)\right|\right]\left(1-\frac{\left\langle\nabla y_{\bar{\gamma}}(x), \nabla y_{\gamma}(x)\right\rangle}{\left|\nabla y_{\bar{\gamma}(x)}\right|\left|\nabla y_{\gamma}(x)\right|}\right)+\frac{g^{2}}{2}\left[\frac{\bar{\gamma}-\gamma}{\bar{\gamma} \gamma}\right]
\end{aligned}
$$

and, therefore,

$$
\frac{1}{\bar{\gamma}-\gamma} \int_{\Omega_{1}} z_{1} \mathrm{~d} x=\frac{g}{2} \int_{\Omega_{1}}\left[\frac{\left|\nabla y_{\bar{\gamma}}\right|-\left|\nabla y_{\gamma}\right|}{\bar{\gamma}-\gamma}\right]\left[1-\frac{\left\langle\nabla y_{\bar{\gamma}}, \nabla y_{\gamma}\right\rangle}{\left|\nabla y_{\bar{\gamma}}\right|\left|\nabla y_{\gamma}\right|}\right] \mathrm{d} x+\frac{g^{2}}{2} \int_{\Omega_{1}} \frac{1}{\bar{\gamma} \gamma} \mathrm{d} x
$$

Next, we estimate the two integrals in (3.26) separately. First, note that, since we are working in $\Omega_{1}$, we have that $\bar{\gamma}\left|\nabla y_{\bar{\gamma}}(x)\right| \geq g$ a.e. Therefore, we obtain the following pointwise estimate in $\Omega_{1}$ :

$$
\begin{aligned}
\left|1-\frac{\left\langle\nabla y_{\bar{\gamma}}(x), \nabla y_{\gamma}(x)\right\rangle}{\left|\nabla y_{\bar{\gamma}}(x)\right|\left|\nabla y_{\gamma}(x)\right|}\right| & \leq\left|1-\frac{\left|\nabla y_{\gamma}(x)\right|}{\left|\nabla y_{\bar{\gamma}}(x)\right|}\right|+\left|\frac{\left|\nabla y_{\gamma}(x)\right|}{\left|\nabla y_{\bar{\gamma}}(x)\right|}-\frac{\left\langle\nabla y_{\bar{\gamma}}(x), \nabla y_{\gamma}(x)\right\rangle}{\left|\nabla y_{\bar{\gamma}}(x)\right|\left|\nabla y_{\gamma}(x)\right|}\right| \\
& \leq \frac{|| \nabla y_{\bar{\gamma}}(x)|-| \nabla y_{\gamma}(x)||}{\left|\nabla y_{\bar{\gamma}}(x)\right|}+\frac{\left|\left\langle\nabla y_{\gamma}(x)-\nabla y_{\bar{\gamma}}(x), \nabla y_{\gamma}(x)\right\rangle\right|}{\left|\nabla y_{\bar{\gamma}}(x)\right|\left|\nabla y_{\gamma}(x)\right|} \\
& \leq \frac{2 \bar{\gamma}}{g}\left|\nabla y_{\bar{\gamma}}(x)-\nabla y_{\gamma}(x)\right| .
\end{aligned}
$$

Therefore, from Cauchy-Schwarz inequality, Theorem 3.2 and (3.27), we have the following estimate

$$
\begin{aligned}
\frac{g}{2} \int_{\Omega_{1}}\left[\frac{\left|\nabla y_{\bar{\gamma}}\right|-\left|\nabla y_{\gamma}\right|}{\bar{\gamma}-\gamma}\right]\left[1-\frac{\left\langle\nabla y_{\bar{\gamma}}, \nabla y_{\gamma}\right\rangle}{\left|\nabla y_{\bar{\gamma}}\right|\left|\nabla y_{\gamma}\right|}\right] \mathrm{d} x & \leq \frac{g}{2|\bar{\gamma}-\gamma|} \int_{\Omega_{1}}\left|\nabla y_{\bar{\gamma}}-\nabla y_{\gamma}\right|\left|1-\frac{\left\langle\nabla y_{\bar{\gamma}}, \nabla y_{\gamma}\right\rangle}{\left|\nabla y_{\bar{\gamma}}\right|\left|\nabla y_{\gamma}\right|}\right| \mathrm{d} x \\
& \leq \frac{\bar{\gamma}}{|\bar{\gamma}-\gamma|} \int_{\Omega_{1}}\left|\nabla y_{\bar{\gamma}}-\nabla y_{\gamma}\right|^{2} \mathrm{~d} x \\
& \leq \frac{\bar{\gamma}}{|\bar{\gamma}-\gamma|}\left\|y_{\bar{\gamma}}-y_{\gamma}\right\|_{H_{0}^{1}}^{2} \leq \bar{\gamma} L^{2}|\bar{\gamma}-\gamma| .
\end{aligned}
$$


Next, we analyze the second expression in the right hand side of (3.26). Since $\mathcal{A}_{\gamma}=\left(\mathcal{A}_{\gamma} \cap \mathcal{A}_{\bar{\gamma}}\right) \cup\left(\mathcal{A}_{\gamma} \backslash \mathcal{A}_{\bar{\gamma}}\right)$, we have that

$$
\frac{g^{2}}{2} \int_{\Omega_{1}} \frac{1}{\bar{\gamma} \gamma} \mathrm{d} x=\frac{g^{2}}{2} \int_{\mathcal{A}_{\gamma}} \frac{1}{\bar{\gamma} \gamma} \mathrm{d} x-\frac{g^{2}}{2} \int_{\mathcal{A}_{\gamma} \backslash \mathcal{A}_{\bar{\gamma}}} \frac{1}{\bar{\gamma} \gamma} \mathrm{d} x .
$$

Further, since $\bar{\gamma}, \gamma \in[M, \infty)$, we obtain

$$
0 \leq \frac{g^{2}}{2} \int_{\mathcal{A}_{\gamma} \backslash \mathcal{A}_{\bar{\gamma}}} \frac{1}{\bar{\gamma} \gamma} \mathrm{d} x \leq \frac{g^{2}}{2 M^{2}} \operatorname{meas}\left(\mathcal{A}_{\gamma} \backslash \mathcal{A}_{\bar{\gamma}}\right),
$$

which, due to Lemma 3.4, implies that

$$
\frac{g^{2}}{2} \int_{\mathcal{A}_{\gamma} \backslash \mathcal{A}_{\bar{\gamma}}} \frac{1}{\bar{\gamma} \gamma} \mathrm{d} x \rightarrow 0, \text { as } \bar{\gamma} \rightarrow \gamma
$$

Thus, from (3.29), (3.30) and the Lebesgue's bounded convergence theorem, we conclude that

$$
\frac{g^{2}}{2} \int_{\Omega_{1}} \frac{1}{\bar{\gamma} \gamma} \mathrm{d} x \rightarrow \frac{g^{2}}{2} \int_{\mathcal{A}_{\gamma}} \frac{1}{\gamma^{2}} \mathrm{~d} x, \text { as } \bar{\gamma} \rightarrow \gamma
$$

Therefore, from (3.26), (3.28) and (3.31), we conclude that

$$
\lim _{\bar{\gamma} \rightarrow \gamma} \frac{1}{\bar{\gamma}-\gamma} \int_{\Omega_{1}} z_{1} \mathrm{~d} x=\frac{1}{2 \gamma^{2}} \int_{\mathcal{A}_{\gamma}} g^{2} \mathrm{~d} x
$$

On $\Omega_{4}$ : We study the limit of $\frac{1}{\bar{\gamma}-\gamma} \int_{\Omega_{4}} z_{4} \mathrm{~d} x$ as $\bar{\gamma} \rightarrow \gamma$. Let us recall that a.e. on $\Omega_{4}, \theta_{\bar{\gamma}}(x)=\theta_{\gamma}(x)=g$, $\psi\left(\nabla y_{\gamma}(x)\right)=\frac{\gamma}{2}\left|\nabla y_{\gamma}(x)\right|^{2}$ and $\psi\left(\nabla y_{\bar{\gamma}}(x)\right)=\frac{\bar{\gamma}}{2}\left|\nabla y_{\bar{\gamma}}(x)\right|^{2}$. Thus, we obtain that

$$
\begin{aligned}
z_{4}(x) & =\left[\frac{\bar{\gamma}}{2}\left|\nabla y_{\bar{\gamma}}(x)\right|^{2}-\frac{\gamma}{2}\left|\nabla y_{\gamma}(x)\right|^{2}-\frac{1}{2}\left\langle\bar{\gamma} \nabla y_{\bar{\gamma}}(x)+\gamma \nabla y_{\gamma}(x), \nabla y_{\bar{\gamma}}(x)-\nabla y_{\gamma}(x)\right\rangle\right] \\
& =\frac{\bar{\gamma}-\gamma}{2}\left\langle\nabla y_{\bar{\gamma}}(x), \nabla y_{\gamma}(x)\right\rangle, \text { a.e., }
\end{aligned}
$$

which implies, since $\mathcal{I}_{\gamma}=\left(\mathcal{I}_{\gamma} \cap \mathcal{I}_{\bar{\gamma}}\right) \cup\left(\mathcal{I}_{\gamma} \backslash \mathcal{I}_{\bar{\gamma}}\right)$, that

$$
\frac{1}{\bar{\gamma}-\gamma} \int_{\Omega_{4}} z_{4} \mathrm{~d} x=\frac{1}{2}\left[\int_{\mathcal{I}_{\gamma}}\left\langle\nabla y_{\bar{\gamma}}, \nabla y_{\gamma}\right\rangle \mathrm{d} x+\int_{\mathcal{I}_{\gamma} \backslash \mathcal{I}_{\bar{\gamma}}}\left\langle\nabla y_{\bar{\gamma}}, \nabla y_{\gamma}\right\rangle \mathrm{d} x\right] .
$$

Let us study the two integrals on the right hand side of (3.33) separately. Theorem 3.2 implies that $y_{\bar{\gamma}} \rightarrow y_{\gamma}$ strongly in $H_{0}^{1}(\Omega)$ as $\bar{\gamma} \rightarrow \gamma$, and, therefore,

$$
\int_{\mathcal{I}_{\gamma}}\left\langle\nabla y_{\bar{\gamma}}, \nabla y_{\gamma}\right\rangle \mathrm{d} x \rightarrow \int_{\mathcal{I}_{\gamma}}\left|\nabla y_{\gamma}\right|^{2} \mathrm{~d} x .
$$

On the other hand, due to Cauchy-Schwarz and Hölder inequalities, and since $\left|\nabla y_{\gamma}(x)\right|<\frac{g}{M}$ and $\left|\nabla y_{\bar{\gamma}}(x)\right|<\frac{g}{M}$ a.e. in $\Omega_{4}$, we obtain that

$$
\left|\int_{\mathcal{I}_{\gamma} \backslash \mathcal{I}_{\bar{\gamma}}}\left\langle\nabla y_{\bar{\gamma}}, \nabla y_{\gamma}\right\rangle \mathrm{d} x\right| \leq \frac{g^{2}}{M} \operatorname{meas}\left(\mathcal{I}_{\bar{\gamma}} \backslash \mathcal{I}_{\gamma}\right)
$$


which, due to Lemma 3.4, imply that

$$
\int_{\mathcal{I}_{\gamma} \backslash \mathcal{I}_{\bar{\gamma}}}\left\langle\nabla y_{\bar{\gamma}}, \nabla y_{\gamma}\right\rangle \mathrm{d} x \rightarrow 0, \text { as } \bar{\gamma} \rightarrow \gamma
$$

Finally, we obtain, from (3.33), (3.34) and (3.35), that

$$
\lim _{\bar{\gamma} \rightarrow \gamma} \frac{1}{\bar{\gamma}-\gamma} \int_{\Omega_{4}} z_{4} \mathrm{~d} x=\frac{1}{2} \int_{\mathcal{I}_{\gamma}}\left|\nabla y_{\gamma}\right|^{2} \mathrm{~d} x
$$

On $\Omega_{2}$ and $\Omega_{3}$ : We study the behavior of $\frac{1}{\bar{\gamma}-\gamma} \int_{\Omega_{2}} z_{2} \mathrm{~d} x$ and $\frac{1}{\bar{\gamma}-\gamma} \int_{\Omega_{3}} z_{3} \mathrm{~d} x$, as $\bar{\gamma} \rightarrow \gamma$. Let us start with $\int_{\Omega_{2}} z_{2} \mathrm{~d} x$. First, note that a.e. in $\Omega_{2}$, we have that $\theta_{\bar{\gamma}}(x)=\bar{\gamma}\left|\nabla y_{\bar{\gamma}}(x)\right|, \theta_{\gamma}(x)=g, \psi\left(\nabla y_{\gamma}(x)\right)=\frac{\gamma}{2}\left|\nabla y_{\gamma}(x)\right|^{2}$ and $\psi\left(\nabla y_{\bar{\gamma}}(x)\right)=g\left|\nabla y_{\bar{\gamma}}(x)\right|-\frac{g^{2}}{2 \bar{\gamma}}$. Thus, we obtain that

$$
z_{2}(x)=g\left|\nabla y_{\bar{\gamma}}(x)\right|-\frac{g^{2}}{2 \bar{\gamma}}-\frac{\gamma}{2}\left|\nabla y_{\gamma}(x)\right|^{2}-\left\langle\frac{g}{2} \frac{\nabla y_{\bar{\gamma}}(x)}{\left|\nabla y_{\bar{\gamma}}(x)\right|}+\frac{\gamma}{2} \nabla y_{\gamma}(x), \nabla y_{\bar{\gamma}}(x)-\nabla y_{\gamma}(x)\right\rangle,
$$

a.e. in $\Omega_{2}$. Moreover, since in $\Omega_{2}$ we have that $\gamma\left|\nabla y_{\gamma}(x)\right|<g$, a.e., and due to the Cauchy-Schwarz inequality, we have the following pointwise estimate:

$$
\begin{aligned}
\left|z_{2}(x)\right| & \leq \frac{g}{2}|| \nabla y_{\bar{\gamma}}(x)\left|-\frac{g}{\gamma}\right|+\frac{1}{2}\left|\frac{g}{\left|\nabla y_{\bar{\gamma}}(x)\right|}-\gamma\right|\left|\left\langle\nabla y_{\bar{\gamma}}(x), \nabla y_{\gamma}(x)\right\rangle\right|+\frac{g^{2}}{2}\left|\frac{\bar{\gamma}-\gamma}{\bar{\gamma} \gamma}\right| \\
& \leq \frac{g}{2}|| \nabla y_{\bar{\gamma}}(x)\left|-\frac{g}{\gamma}\right|+\frac{\gamma\left|\nabla y_{\gamma}(x)\right|}{2}|| \nabla y_{\bar{\gamma}}(x)\left|-\frac{g}{\gamma}\right|+\frac{g^{2}}{2}\left|\frac{\bar{\gamma}-\gamma}{\bar{\gamma} \gamma}\right| \\
& <g|| \nabla y_{\bar{\gamma}}(x)\left|-\frac{g}{\gamma}\right|+\frac{g^{2}}{2}\left|\frac{\bar{\gamma}-\gamma}{\bar{\gamma} \gamma}\right|
\end{aligned}
$$

a.e. in $\Omega_{2}$. Then, we divide the analysis in two cases:

(i) $\left|\nabla y_{\bar{\gamma}}(x)\right|-\frac{g}{\gamma} \geq 0$ : Since in $\Omega_{2}$ we have that $\left|\nabla y_{\gamma}(x)\right|<\frac{g}{\gamma}$ a.e., and due to Theorem 3.2 and (3.37), we obtain that

$$
\begin{aligned}
\frac{1}{|\bar{\gamma}-\gamma|} \int_{\left\{\left|\nabla y_{\bar{\gamma}}\right| \geq \frac{g}{\gamma}\right\}}\left|z_{2}\right| \mathrm{d} x & \leq g|\bar{\gamma}-\gamma|^{-1} \int_{\Omega_{2}}\left|\nabla y_{\bar{\gamma}}-\nabla y_{\gamma}\right| \mathrm{d} x+\frac{g^{2}}{2 \bar{\gamma} \gamma} \operatorname{meas}\left(\Omega_{2}\right) \\
& \leq g|\bar{\gamma}-\gamma|^{-1} \operatorname{meas}\left(\Omega_{2}\right)^{1 / 2}\left\|\nabla y_{\bar{\gamma}}-\nabla y_{\gamma}\right\|_{H_{0}^{1}}+\frac{g^{2}}{2 \bar{\gamma} \gamma} \operatorname{meas}\left(\Omega_{2}\right) \\
& \leq g L \operatorname{meas}\left(\Omega_{2}\right)^{1 / 2}+\frac{g^{2}}{2 M^{2}} \operatorname{meas}\left(\Omega_{2}\right) .
\end{aligned}
$$

(ii) $\left|\nabla y_{\bar{\gamma}}(x)\right|-\frac{g}{\gamma}<0$ : Since in $\Omega_{2}$ we have that $\left|\nabla y_{\bar{\gamma}}(x)\right| \geq \frac{g}{\bar{\gamma}}$ a.e., we have that

$$
\begin{aligned}
\frac{1}{|\bar{\gamma}-\gamma|} \int_{\left\{|\nabla y \bar{\gamma}|<\frac{g}{\gamma}\right\}}\left|z_{2}\right| \mathrm{d} x & \leq g|\bar{\gamma}-\gamma|^{-1} \int_{\Omega_{2}}\left|\frac{g}{\gamma}-\frac{g}{\bar{\gamma}}\right| \mathrm{d} x+\frac{g^{2}}{2 \bar{\gamma} \gamma} \operatorname{meas}\left(\Omega_{2}\right) \\
& \leq \frac{3 g^{2}}{2 M^{2}} \operatorname{meas}\left(\Omega_{2}\right) .
\end{aligned}
$$

Consequently, (3.37), (3.38), (3.39) and Lemma 3.4 imply that

$$
\lim _{\bar{\gamma} \rightarrow \gamma} \frac{1}{\bar{\gamma}-\gamma} \int_{\Omega_{2}}\left|z_{2}\right| \mathrm{d} x=0
$$

Analogously, we conclude that

$$
\lim _{\bar{\gamma} \rightarrow \gamma} \frac{1}{\bar{\gamma}-\gamma} \int_{\Omega_{3}}\left|z_{3}\right| \mathrm{d} x=0 .
$$

Then, the result follows from (3.32), (3.36), (3.40) and (3.41). 
Proposition 3.8. Let $\gamma \in[M, \infty)$. The function $\gamma \mapsto V(\gamma)$ is twice differentiable at $\gamma$, with its second derivative given by

$$
\ddot{V}(\gamma)=-\frac{1}{\gamma^{3}} \int_{\mathcal{A}_{\gamma}} g^{2} \mathrm{~d} x+\int_{\mathcal{I}_{\gamma}}\left\langle\nabla y_{\gamma}, \nabla \dot{y}_{\gamma}\right\rangle \mathrm{d} x,
$$

where $\dot{y}_{\gamma}$ is defined in Proposition 3.6. Moreover, $\ddot{V}(\gamma) \leq 0$, for all $\gamma \in[M, \infty)$.

Proof. Let us first prove (3.42). Since $\left|q_{\gamma}(x)\right|=g$, a.e. in $\mathcal{A}_{\gamma}$, and $\left|q_{\gamma}(x)\right|=\gamma\left|\nabla y_{\gamma}(x)\right|$ a.e. in $\mathcal{I}_{\gamma}$, we can write

$$
\dot{V}(\gamma)=\frac{1}{2 \gamma^{2}} \int_{\Omega}\left|q_{\gamma}\right|^{2} \mathrm{~d} x .
$$

From (3.43) we conclude that

$$
\dot{V}(\bar{\gamma})-\dot{V}(\gamma)=\frac{1}{2 \bar{\gamma}^{2}} \int_{\Omega}\left|q_{\bar{\gamma}}\right|^{2} \mathrm{~d} x-\frac{1}{2 \gamma^{2}} \int_{\Omega}\left|q_{\gamma}\right|^{2} \mathrm{~d} x .
$$

We are concerned with the limit $\lim _{\bar{\gamma} \rightarrow \gamma} \frac{\dot{V}(\bar{\gamma})-\dot{V}(\gamma)}{\bar{\gamma}-\gamma}$. We introduce the notation $\mathrm{I}_{j}=\frac{1}{2 \bar{\gamma}^{2}} \int_{\Omega_{j}}\left|q_{\bar{\gamma}}\right|^{2} \mathrm{~d} x-$ $\frac{1}{2 \gamma^{2}} \int_{\Omega_{j}}\left|q_{\gamma}\right|^{2} \mathrm{~d} x, j=1, \ldots, 4$, where the sets $\Omega_{j}, j=1, \ldots, 4$ are defined as in Proposition 3.7, and we analyze the integrals $\mathrm{I}_{j}$ separately.

On $\Omega_{1}\left(\lim _{\bar{\gamma} \rightarrow \gamma} \frac{1}{\bar{\gamma}-\gamma} \mathrm{I}_{1}\right)$ : Let us start by recalling that a.e. on $\Omega_{1}$, we have that $\left|q_{\bar{\gamma}}(x)\right|=\left|q_{\gamma}(x)\right|=g$. Thus, since $\mathcal{A}_{\gamma}=\left(\mathcal{A}_{\gamma} \cap \mathcal{A}_{\bar{\gamma}}\right) \cup\left(\mathcal{A}_{\gamma} \backslash \mathcal{A}_{\bar{\gamma}}\right)$, we get

$$
\begin{aligned}
(\bar{\gamma}-\gamma)^{-1} \mathrm{I}_{1} & =(\bar{\gamma}-\gamma)^{-1} \int_{\Omega_{1}} \frac{g^{2}\left(\gamma^{2}-\bar{\gamma}^{2}\right)}{2 \bar{\gamma}^{2} \gamma^{2}} \mathrm{~d} x \\
& =-\int_{\mathcal{A}_{\gamma}} \frac{g^{2}(\gamma+\bar{\gamma})}{2 \bar{\gamma}^{2} \gamma^{2}} \mathrm{~d} x-\int_{\mathcal{A}_{\gamma} \backslash \mathcal{A}_{\bar{\gamma}}} \frac{g^{2}(\gamma+\bar{\gamma})}{2 \bar{\gamma}^{2} \gamma^{2}} \mathrm{~d} x
\end{aligned}
$$

Since $\bar{\gamma}, \gamma \in[M, \infty)$, we get that

$$
0 \leq \int_{\mathcal{A}_{\gamma} \backslash \mathcal{A}_{\bar{\gamma}}} \frac{g^{2}(\gamma+\bar{\gamma})}{2 \bar{\gamma}^{2} \gamma^{2}} \mathrm{~d} x \leq \frac{g^{2}(\gamma+\bar{\gamma})}{2 M^{4}} \operatorname{meas}\left(\mathcal{A}_{\gamma} \backslash \mathcal{A}_{\bar{\gamma}}\right)
$$

which, due to Lemma 3.4, implies that

$$
\int_{\mathcal{A}_{\gamma} \backslash \mathcal{A}_{\bar{\gamma}}} \frac{g^{2}(\gamma+\bar{\gamma})}{2 \bar{\gamma}^{2} \gamma^{2}} \mathrm{~d} x \rightarrow 0 \text { as } \bar{\gamma} \rightarrow \gamma
$$

Thus, (3.44), Lemma 3.4 and the Lebesgue's bounded convergence theorem imply that

$$
\lim _{\bar{\gamma} \rightarrow \gamma}(\bar{\gamma}-\gamma)^{-1} \mathrm{I}_{1}=-\frac{1}{\gamma^{3}} \int_{\mathcal{A}_{\gamma}} g^{2} \mathrm{~d} x .
$$

On $\Omega_{4}\left(\lim _{\bar{\gamma} \rightarrow \gamma} \frac{1}{\bar{\gamma}-\gamma} \mathrm{I}_{4}\right)$ : First, note that $\left|q_{\gamma}(x)\right|=\gamma\left|\nabla y_{\gamma}(x)\right|$ and $\left|q_{\bar{\gamma}}(x)\right|=\bar{\gamma}\left|\nabla y_{\bar{\gamma}}(x)\right|$ a.e. on $\Omega_{4}$. Thus, since $\mathcal{I}_{\gamma}=\left(\mathcal{I}_{\gamma} \cap \mathcal{I}_{\bar{\gamma}}\right) \cup\left(\mathcal{I}_{\gamma} \backslash \mathcal{I}_{\bar{\gamma}}\right)$, we obtain that

$$
(\bar{\gamma}-\gamma)^{-1} \mathrm{I}_{4}=\frac{1}{2(\bar{\gamma}-\gamma)} \int_{\mathcal{I}_{\gamma}}\left[\left|\nabla y_{\bar{\gamma}}\right|^{2}-\left|\nabla y_{\gamma}\right|^{2}\right] \mathrm{d} x-\frac{1}{2(\bar{\gamma}-\gamma)} \int_{\mathcal{I}_{\gamma} \backslash \mathcal{I}_{\bar{\gamma}}}\left[\left|\nabla y_{\bar{\gamma}}\right|^{2}-\left|\nabla y_{\gamma}\right|^{2}\right] \mathrm{d} x
$$

Next, let us analyze the two integrals of the right hand side of (3.46) separately. 
(i) $\lim _{\bar{\gamma} \rightarrow \gamma} \frac{1}{2(\bar{\gamma}-\gamma)} \int_{\mathcal{I}_{\gamma}}\left[\left|\nabla y_{\bar{\gamma}}\right|^{2}-\left|\nabla y_{\gamma}\right|^{2}\right] \mathrm{d} x$ : Note that

$$
\frac{1}{\bar{\gamma}-\gamma} \int_{\mathcal{I}_{\gamma}}\left[\left|\nabla y_{\bar{\gamma}}\right|^{2}-\left|\nabla y_{\gamma}\right|^{2}\right] \mathrm{d} x=\underbrace{\int_{\mathcal{I}_{\gamma}}\left\langle\nabla y_{\bar{\gamma}}, \nabla\left(\frac{y_{\bar{\gamma}}-y_{\gamma}}{\bar{\gamma}-\gamma}\right)\right\rangle+\left\langle\nabla y_{\gamma}, \nabla\left(\frac{y_{\bar{\gamma}}-y_{\gamma}}{\bar{\gamma}-\gamma}\right)\right\rangle \mathrm{d} x}_{:=J_{1}},
$$

which, implies that

$$
\begin{aligned}
\left|J_{1}-2 \int_{\mathcal{I}_{\gamma}}\left\langle\nabla y_{\gamma}, \nabla \dot{y}_{\gamma}\right\rangle \mathrm{d} x\right| \leq & \left|\int_{\mathcal{I}_{\gamma}}\left\langle\nabla y_{\bar{\gamma}}, \nabla\left(\frac{y_{\bar{\gamma}}-y_{\gamma}}{\bar{\gamma}-\gamma}\right)\right\rangle-\left\langle\nabla y_{\gamma}, \nabla \dot{y}_{\gamma}\right\rangle \mathrm{d} x\right| \\
& +\left|\int_{\mathcal{I}_{\gamma}}\left\langle\nabla y_{\gamma}, \nabla\left(\frac{y_{\bar{\gamma}}-y_{\gamma}}{\bar{\gamma}-\gamma}\right)-\nabla \dot{y}_{\gamma}\right\rangle \mathrm{d} x\right| .
\end{aligned}
$$

Next, we separately analyze the two terms in the right hand side of the inequality above. Since $\dot{y}_{\gamma}$ is an accumulation point of $\frac{y_{\bar{\gamma}}-y_{\gamma}}{\bar{\gamma}-\gamma}$ in $H_{0}^{1}(\Omega)$ as $\bar{\gamma} \rightarrow \gamma$ (see Rem. 3.2 and Thm. 3.6), we have that

$$
\int_{\mathcal{I}_{\gamma}}\left\langle\nabla y_{\gamma}, \nabla\left(\frac{y_{\bar{\gamma}}-y_{\gamma}}{\bar{\gamma}-\gamma}\right)-\nabla \dot{y}_{\gamma}\right\rangle \mathrm{d} x \rightarrow 0 \text { as } \bar{\gamma} \rightarrow \gamma
$$

On the other hand, we have that

$$
\begin{aligned}
\left|\int_{\mathcal{I}_{\gamma}}\left\langle\nabla y_{\bar{\gamma}}, \nabla\left(\frac{y_{\bar{\gamma}}-y_{\gamma}}{\bar{\gamma}-\gamma}\right)\right\rangle-\left\langle\nabla y_{\gamma}, \nabla \dot{y}_{\gamma}\right\rangle \mathrm{d} x\right| \leq \\
\left|\int_{\mathcal{I}_{\gamma}}\left\langle\nabla y_{\gamma}, \nabla\left(\frac{y_{\bar{\gamma}}-y_{\gamma}}{\bar{\gamma}-\gamma}\right)-\nabla \dot{y}_{\gamma}\right\rangle \mathrm{d} x\right|+\int_{\mathcal{I}_{\gamma}}\left|\left\langle\nabla y_{\bar{\gamma}}-\nabla y_{\gamma}, \nabla\left(\frac{y_{\bar{\gamma}}-y_{\gamma}}{\bar{\gamma}-\gamma}\right)\right\rangle\right| \mathrm{d} x,
\end{aligned}
$$

which, since $\dot{y}_{\gamma}$ is an accumulation point of $\frac{y_{\bar{\gamma}}-y_{\gamma}}{\bar{\gamma}-\gamma}$ in $H_{0}^{1}(\Omega)$ as $\bar{\gamma} \rightarrow \gamma$, implies that

$$
\left|\int_{\mathcal{I}_{\gamma}}\left\langle\nabla y_{\gamma}, \nabla\left(\frac{y_{\bar{\gamma}}-y_{\gamma}}{\bar{\gamma}-\gamma}\right)\right\rangle-\left\langle\nabla y_{\gamma}, \nabla \dot{y}_{\gamma}\right\rangle \mathrm{d} x\right| \rightarrow 0 \text { as } \bar{\gamma} \rightarrow \gamma
$$

Furthermore, Theorem 3.2 yields that

$$
\int_{\mathcal{I}_{\gamma}}\left|\left\langle\nabla y_{\bar{\gamma}}-\nabla y_{\gamma}, \nabla\left(\frac{y_{\bar{\gamma}}-y_{\gamma}}{\bar{\gamma}-\gamma}\right)\right\rangle\right| \mathrm{d} x=|\bar{\gamma}-\gamma|^{-1}\left\|\nabla y_{\bar{\gamma}}-\nabla y_{\gamma}\right\|_{H_{0}^{1}}^{2} \leq L^{2}|\bar{\gamma}-\gamma| .
$$

Consequently, (3.47), (3.48) and (3.49) imply

$$
\lim _{\bar{\gamma} \rightarrow \gamma} \frac{1}{2(\bar{\gamma}-\gamma)} \int_{\mathcal{I}_{\gamma}}\left[\left|\nabla y_{\bar{\gamma}}\right|^{2}-\left|\nabla y_{\gamma}\right|^{2}\right] \mathrm{d} x=\int_{\mathcal{I}_{\gamma}}\left\langle\nabla y_{\gamma}, \dot{y}_{\gamma}\right\rangle \mathrm{d} x
$$

(ii) $\lim _{\bar{\gamma} \rightarrow \gamma} \frac{1}{2(\bar{\gamma}-\gamma)} \int_{\mathcal{I}_{\gamma} \backslash \mathcal{I}_{\bar{\gamma}}}\left[\left|\nabla y_{\bar{\gamma}}\right|^{2}-\left|\nabla y_{\gamma}\right|^{2}\right] \mathrm{d} x$ : First note that

$$
\left.\int_{\mathcal{I}_{\gamma} \backslash \mathcal{I}_{\bar{\gamma}}}|| \nabla y_{\bar{\gamma}}\right|^{2}-\left|\nabla y_{\gamma}\right|^{2}\left|\mathrm{~d} x \leq \int_{\mathcal{I}_{\gamma} \backslash \mathcal{I}_{\bar{\gamma}}}\right| \nabla y_{\gamma}|| \nabla y_{\bar{\gamma}}-\nabla y_{\gamma}\left|\mathrm{d} x+\int_{\mathcal{I}_{\gamma} \backslash \mathcal{I}_{\bar{\gamma}}}\right| \nabla y_{\bar{\gamma}}|| \nabla y_{\bar{\gamma}}-\nabla y_{\gamma} \mid \mathrm{d} x .
$$


Therefore, from Theorem 3.2, Remark 2.4 and Hölder inequality, we have that

$$
\begin{aligned}
\left.\int_{\mathcal{I}_{\gamma} \backslash \mathcal{I}_{\bar{\gamma}}}|| \nabla y_{\bar{\gamma}}\right|^{2}-\left|\nabla y_{\gamma}\right|^{2} \mid \mathrm{d} x & \leq \operatorname{meas}\left(\mathcal{I}_{\gamma} \backslash \mathcal{I}_{\bar{\gamma}}\right)^{1 / 4}\left(\left\|\nabla y_{\bar{\gamma}}\right\|_{\mathbf{L}^{4}}+\left\|\nabla y_{\gamma}\right\|_{\mathbf{L}^{4}}\right)\left\|\nabla y_{\bar{\gamma}}-\nabla y_{\gamma}\right\|_{H_{0}^{1}} \\
& \leq L|\bar{\gamma}-\gamma| 2 K \operatorname{meas}\left(\mathcal{I}_{\gamma} \backslash \mathcal{I}_{\bar{\gamma}}\right)^{1 / 4}
\end{aligned}
$$

and then, from Lemma 3.4, we conclude that

$$
\lim _{\bar{\gamma} \rightarrow \gamma} \frac{1}{2(\bar{\gamma}-\gamma)} \int_{\mathcal{I}_{\gamma} \backslash \mathcal{I}_{\bar{\gamma}}}\left[\left|\nabla y_{\bar{\gamma}}\right|^{2}-\left|\nabla y_{\gamma}\right|^{2}\right] \mathrm{d} x=0
$$

Finally, (3.46), (3.50) and (3.51) imply that

$$
\lim _{\bar{\gamma} \rightarrow \gamma} \frac{1}{\bar{\gamma}-\gamma} \mathrm{I}_{4}=\int_{\mathcal{I}_{\gamma}}\left\langle\nabla y_{\gamma}, \nabla \dot{y}_{\gamma}\right\rangle \mathrm{d} x
$$

On $\Omega_{2}$ and $\Omega_{3}$ : We analyze the limits $\lim _{\bar{\gamma} \rightarrow \gamma} \frac{1}{\bar{\gamma}-\gamma} \mathrm{I}_{2}$ and $\lim _{\bar{\gamma} \rightarrow \gamma} \frac{1}{\bar{\gamma}-\gamma} \mathrm{I}_{3}$. We start by analyzing $\lim _{\bar{\gamma} \rightarrow \gamma} \frac{1}{\bar{\gamma}-\gamma} \mathrm{I}_{2}$. Thus, we recall that in $\Omega_{2}$, we have that $\left|\nabla y_{\bar{\gamma}}(x)\right| \geq \frac{g}{\bar{\gamma}}$. Then, from Theorem 3.2, Remark 2.4 and Hölder inequality, we conclude that

$$
\left|\mathrm{I}_{2}\right| \leq\left.\frac{1}{2} \int_{\Omega_{2}}\left|\frac{g^{2}}{\bar{\gamma}^{2}}-\right| \nabla y_{\gamma}\right|^{2}\left|\mathrm{~d} x \leq \frac{1}{2} \int_{\Omega_{2}}\right|\left|\nabla y_{\bar{\gamma}}\right|^{2}-\left|\nabla y_{\gamma}\right|^{2} \mid \mathrm{d} x \leq L K \operatorname{meas}\left(\Omega_{2}\right)^{1 / 4}
$$

which, due to Lemma 3.4, implies that

$$
\lim _{\bar{\gamma} \rightarrow \gamma} \frac{1}{\bar{\gamma}-\gamma}\left|\mathrm{I}_{2}\right|=0
$$

Analogously, we conclude that

$$
\lim _{\bar{\gamma} \rightarrow \gamma} \frac{1}{\bar{\gamma}-\gamma}\left|\mathrm{I}_{3}\right|=0
$$

Finally, (3.45), (3.52), (3.53), and (3.54) imply (3.43).

Now, we prove that $\ddot{V}(\gamma) \leq 0$. Using $v=\dot{y}_{\gamma} \chi_{\mathcal{I}_{\gamma}}$ in (3.21), we obtain that

$$
a\left(\dot{y}_{\gamma}, \dot{y}_{\gamma} \chi_{\mathcal{I}_{\gamma}}\right)+\left(\nabla y_{\gamma}, \nabla \dot{y}_{\gamma} \chi_{\mathcal{I}_{\gamma}}\right)_{\mathbf{L}^{2}(\Omega)}+\gamma\left(\nabla \dot{y}_{\gamma} \chi_{\mathcal{I}_{\gamma}}, \nabla \dot{y}_{\gamma} \chi_{\mathcal{I}_{\gamma}}\right)_{\mathbf{L}^{2}(\Omega)}=0
$$

which implies that

$$
(\mu+\gamma) \int_{\mathcal{I}_{\gamma}}\left|\nabla \dot{y}_{\gamma}\right|^{2} \mathrm{~d} x=-\int_{\mathcal{I}_{\gamma}}\left\langle\nabla y_{\gamma}, \nabla \dot{y}_{\gamma}\right\rangle \mathrm{d} x .
$$

Thus, we can easily conclude that

$$
\int_{\mathcal{I}_{\gamma}}\left\langle\nabla y_{\gamma}, \nabla \dot{y}_{\gamma}\right\rangle \mathrm{d} x \leq 0
$$

which yields that

$$
\ddot{V}(\gamma)=-\frac{1}{\gamma^{3}} \int_{\mathcal{A}_{\gamma}} g^{2} \mathrm{~d} x+\int_{\mathcal{I}_{\gamma}}\left\langle\nabla y_{\gamma}, \nabla \dot{y}_{\gamma}\right\rangle \mathrm{d} x \leq 0 .
$$




\subsection{Model functions and path-following algorithm}

In this section, following [14], we propose model functions which approximate the value functional $V(\gamma)$ and share some of its qualitative properties. These model functions will then be used for the development of the path following algorithm.

From Theorem 3.2 and Propositions 3.7 and 3.8, it follows that $\gamma \mapsto V(\gamma), \gamma \in[M, \infty)$ is a monotonically increasing and concave function. We then propose the model functions

$$
m(\gamma)=C_{1}-\frac{C_{2}}{\mu+\gamma}-\frac{G}{\gamma}
$$

with $C_{1} \in \mathbb{R}, C_{2} \geq 0$ and $G \geq 0$, which share the main qualitative properties of $V(\gamma)$, i.e., $\dot{m}(\gamma) \geq 0$ and $\ddot{m}(\gamma) \leq 0$.

To motivate the introduction of these model functions, let us take the test function $v=y_{\gamma} \chi_{\mathcal{I}_{\gamma}}$ in (3.21). We get that

$$
a\left(\dot{y}_{\gamma}, y_{\gamma} \chi_{\mathcal{I}_{\gamma}}\right)+\left(\nabla y_{\gamma} \chi_{\mathcal{I}_{\gamma}}, \nabla y_{\gamma} \chi_{\mathcal{I}_{\gamma}}\right)_{\mathbf{L}^{2}(\Omega)}+\gamma\left(\nabla \dot{y}_{\gamma} \chi_{\mathcal{I}_{\gamma}}, \nabla y_{\gamma} \chi_{\mathcal{I}_{\gamma}}\right)_{\mathbf{L}^{2}(\Omega)}=0 .
$$

From the definition of $a(\cdot, \cdot)$, we obtain that

$$
(\mu+\gamma)\left(\nabla \dot{y}_{\gamma}, \nabla y_{\gamma} \chi_{\mathcal{I}_{\gamma}}\right)_{\mathbf{L}^{2}(\Omega)}+\int_{\mathcal{I}_{\gamma}}\left|\nabla y_{\gamma}\right|^{2} \mathrm{~d} x=0 .
$$

Consequently, by using Propositions 3.7 and 3.8, we obtain

$$
(\mu+\gamma)\left[\ddot{V}(\gamma)+\frac{1}{\gamma^{3}} \int_{\mathcal{A}_{\gamma}} g^{2} \mathrm{~d} x\right]+2 \dot{V}(\gamma)-\frac{1}{\gamma^{2}} \int_{\mathcal{A}_{\gamma}} g^{2} \mathrm{~d} x=0,
$$

which implies that

$$
(\mu+\gamma) \ddot{V}(\gamma)+2 \dot{V}(\gamma)+\mu \gamma^{-3} \int_{\mathcal{A}_{\gamma}} g^{2} \mathrm{~d} x=0 .
$$

Note that $\int_{\mathcal{A}_{\gamma}} g^{2}$ is a function of $\gamma$ which is uniformly bounded from above by $g^{2}$ meas $(\Omega)$.

Replacing $V$ by $m$ and the $\gamma$ dependent term $\int_{\mathcal{A}_{\gamma}} g^{2}$ by $2 G$, we obtain the differential equation

$$
(\mu+\gamma) \ddot{m}(\gamma)+2 \dot{m}(\gamma)+2 \gamma^{-3} \mu G=0,
$$

whose solutions are the family of functions given by (3.55).

In order to determine $C_{1}, C_{2}$ and $G$, we fix a reference value $\gamma_{r}>0, \gamma_{r} \neq \gamma$, for which the value $V\left(\gamma_{r}\right)$ is known. Then, we use the following conditions

$$
m(\gamma)=V(\gamma), m\left(\gamma_{r}\right)=V\left(\gamma_{r}\right), \dot{m}(\gamma)=\dot{V}(\gamma) .
$$

Solving the resulting system of nonlinear equations

$$
\left\{\begin{aligned}
C_{1}-\frac{C_{2}}{\mu+\gamma}-\frac{G}{\gamma} & =V(\gamma) \\
C_{1}-\frac{C_{2}}{\mu+\gamma_{r}}-\frac{G}{\gamma_{r}} & =V\left(\gamma_{r}\right), \\
\frac{C_{2}}{(\mu+\gamma)^{2}}+\frac{G}{\gamma^{2}} & =\dot{V}(\gamma),
\end{aligned}\right.
$$


we obtain that

$$
G=\frac{\gamma \gamma_{r}\left(V\left(\gamma_{r}\right)-V(\gamma)\right)}{\gamma_{r}-\gamma}-\frac{\gamma_{r} \gamma^{2} \vartheta(\mu+\gamma)}{\mu\left(\gamma-\gamma_{r}\right)}
$$

where $\vartheta:=\frac{1}{\gamma-\gamma_{r}}\left[\dot{V}(\gamma)-\frac{\gamma_{r}\left(V\left(\gamma_{r}\right)-V(\gamma)\right)}{\gamma\left(\gamma_{r}-\gamma\right)}\right]$. Consequently, the parameters $C_{1}$ and $C_{2}$ are given by

$$
C_{2}=(\mu+\gamma)^{2}\left(\dot{V}(\gamma)-\frac{G}{\gamma^{2}}\right), \quad C_{1}=V(\gamma)+\frac{C_{2}}{\mu+\gamma}+\frac{G}{\gamma} .
$$

Once we have determined the values of the coefficients of the model, we are able to propose the updating strategy for $\gamma$. Let $\left\{\tau_{k}\right\}$ satisfy $\tau_{k} \in(0,1)$ for all $k \in \mathbb{N}$ and $\tau_{k} \downarrow 0$ as $k \rightarrow \infty$, and assume that $V\left(\gamma_{k}\right)$ is available. Following [14], the idea is to have a superlinear rate of convergence for our algorithm, i.e., given $\gamma_{k}$, the update value $\gamma_{k+1}$ should ideally satisfy

$$
\left|\bar{V}-V\left(\gamma_{k+1}\right)\right| \leq \tau_{k}\left|\bar{V}-V\left(\gamma_{k}\right)\right|
$$

where $\bar{V}:=\lim _{\gamma \rightarrow \infty} V(\gamma)$. Since $\bar{V}$ and $V\left(\gamma_{k+1}\right)$ are unknowns, we approximate these values by $\lim _{\gamma_{k} \rightarrow \infty} m\left(\gamma_{k}\right)$ and $m\left(\gamma_{k+1}\right)$, respectively. Hereafter, we use the notation $C_{1, k}, C_{2, k}$ and $G_{k}$ for the coefficients on the model function (3.55) related with each $\gamma_{k}$.

Further, note that $\lim _{\gamma_{k} \rightarrow \infty} m\left(\gamma_{k}\right)=C_{1, k}$. Thus, (3.60) is replaced by

$$
\left|C_{1, k}-m\left(\gamma_{k+1}\right)\right| \leq \tau_{k}\left|C_{1, k}-m\left(\gamma_{k}\right)\right|
$$

Calling $\beta_{k}:=\tau_{k}\left|C_{1, k}-m\left(\gamma_{k}\right)\right|$ and solving the equation $C_{1, k}-m\left(\gamma_{k+1}\right)=\beta_{k}$, we obtain that

$$
\gamma_{k+1}=\frac{D_{k}}{2}+\sqrt{\frac{D_{k}^{2}}{4}+\frac{\mu G_{k}}{\beta_{k}}}
$$

where $D_{k}=\frac{\left(C_{2, k}+G_{k}\right)}{\beta_{k}}-\mu$.

Next, we write a path-following algorithm which uses the update strategy for $\gamma$, given by (3.62).

\section{Algorithm PF.}

1. Select $\gamma_{r}$ and compute $V\left(\gamma_{r}\right)$. Choose $\gamma_{0}>\max \left(1, \gamma_{r}\right)$ and set $k=0$.

2. Solve

$$
\begin{cases}a\left(y_{\gamma_{k}}, v\right)+\left(q_{\gamma_{k}}, v\right)_{\mathbf{L}^{2}(\Omega)}-(f, v)_{2}=0, & \text { for all } v \in H_{0}^{1}(\Omega) \\ \max \left(g, \gamma_{k}\left|\nabla y_{\gamma_{k}}(x)\right|\right) q_{\gamma_{k}}(x)=g \gamma_{k} \nabla y_{\gamma_{k}}(x), & \text { a.e. in } \Omega .\end{cases}
$$

3. Compute $V\left(\gamma_{k}\right), \dot{V}\left(\gamma_{k}\right)$ and update $\gamma_{k}$ by using

$$
\gamma_{k+1}=\frac{D_{k}}{2}+\sqrt{\frac{D_{k}^{2}}{4}+\frac{\mu G_{k}}{\beta_{k}}}
$$

4. Stop, or set $k:=k+1$ and go to step 2 .

\section{Semismooth Newton method}

In this section we state an algorithm for the efficient solution of (3.63). Since no smoothing operation takes place in the complementary function in (3.63), it is not possible to get Newton differentiability in infinite 
dimensions (see [27], Sect. 3.3). Therefore, we consider a discretized version of system (3.63), and propose a semismooth Newton method to solve this problem.

Specifically, we state a primal-dual scheme to solve system (3.63) and prove local superlinear convergence of the method. By involving the primal and the dual variables in the same algorithm, we compute the solutions to the discrete versions of $\left(\mathcal{P}_{\gamma}\right)$ and $\left(\mathcal{P}_{\gamma}^{*}\right)$ simultaneously. The algorithm proposed is a particular case of the Newton type algorithms developed in [16].

Let us introduce the definition of Newton differentiability

Definition 4.1. Let $X$ and $Z$ be two Banach spaces. The function $F: X \rightarrow Z$ is called Newton differentiable if there exists a family of generalized derivatives $G: X \rightarrow \mathcal{L}(X, Z)$ such that

$$
\lim _{h \rightarrow 0} \frac{1}{\|h\|_{X}}\|F(x+h)-F(x)-G(x+h) h\|_{Z}=0 .
$$

Throughout this section we denote discretized quantities by superscript $h$. For a vector $v \in \mathbb{R}^{n}$ we denote by $D(v):=\operatorname{diag}(v)$ the $n \times n$-diagonal matrix with diagonal entries $v_{i}$. Besides that, we denote by $\star$ the Hadamard product of vectors, i.e., $v \star w:=\left(v_{1} w_{1}, \ldots, v_{n} w_{n}\right)^{\top}$.

We use a finite element approximation of system (3.63) and consider the spaces

$$
\begin{aligned}
& V_{h}:=\left\{\eta \in C(\bar{\Omega}): \eta_{\left.\right|_{T}} \in \Pi_{1}, \forall T \in \mathcal{T}^{h}\right\}, \\
& W_{h}:=\left\{q^{h}:=\left(q_{1}^{h}, q_{2}^{h}\right)^{\top} \in \mathbf{L}^{2}(\Omega):\left.q_{1}^{h}\right|_{T},\left.q_{2}^{h}\right|_{T} \in \Pi_{0}, \forall T \in \mathcal{T}^{h}\right\},
\end{aligned}
$$

to approximate the velocity $y^{h}$ and the multiplier $q^{h}$, respectively. Here, $\Pi_{k}$ denotes the set of polynomials of degree less or equal than $k$ and $\mathcal{T}^{h}$ denotes a regular triangulation of $\Omega$. Thus, the discrete analogous of (3.63) is given by

$$
\left\{\begin{array}{l}
A_{\mu}^{h} \vec{y}+B^{h} \vec{q}-f^{h}=0 \\
\max \left(g e^{h}, \gamma \xi\left(\nabla^{h} \vec{y}\right)\right) \star \vec{q}-\gamma g \nabla^{h} \vec{y}=0, \text { for } \gamma>0,
\end{array}\right.
$$

where $A_{\mu}^{h} \in \mathbb{R}^{n \times n}$ is the stiffness matrix, $e^{h} \in \mathbb{R}^{2 m}$ is the vector of all ones and $B^{h} \in \mathbb{R}^{n \times 2 m}$ is obtained in the usual way, from the bilinear form $(\cdot, \nabla \cdot)_{\mathbf{L}^{2}(\Omega)}$ and the basis functions of $V_{h}$ and $W_{h}$. Here, $y^{h} \in \mathbb{R}^{n}$ and $q^{h} \in \mathbb{R}^{2 m}$ are the solution coefficients of the approximated regularized primal and dual solutions $y_{\gamma}^{h} \in V_{h}$ and $q_{\gamma}^{h} \in W_{h}$, respectively. Further, we construct the right hand side $f^{h}$ using the basis functions $\varphi_{i} \in V_{h}, i=1, \ldots, n$, (see [1], Sect. 6). The discrete version of the gradient is given by

$$
\nabla^{h}:=\left(\begin{array}{c}
\partial_{1}^{h} \\
\partial_{2}^{h}
\end{array}\right) \in \mathbb{R}^{2 m \times n}
$$

where $\partial_{1}^{h}:=\left.\frac{\partial \varphi_{i}(x)}{\partial x_{1}}\right|_{T_{k}}$ and $\partial_{2}^{h}:=\left.\frac{\partial \varphi_{i}(x)}{\partial x_{2}}\right|_{T_{k}}$, for $i=1, \ldots, n ; k=1, \ldots, m$. Note that $\left.\frac{\partial \varphi_{i}(x)}{\partial x_{1}}\right|_{T_{k}}$ and $\left.\frac{\partial \varphi_{i}(x)}{\partial x_{2}}\right|_{T_{k}}$ are the constant values of $\frac{\partial \varphi_{i}(x)}{\partial x_{1}}$ and $\frac{\partial \varphi_{i}(x)}{\partial x_{2}}$ in each triangle $T_{k}$, respectively. Consequently, we obtain that $\nabla^{h} \vec{y}=\nabla y^{h}(x)$.

Hereafter, the matrix $A_{\mu}^{h}$ is assumed to be symmetric and positive definite. The function $\xi: \mathbb{R}^{2 m} \rightarrow \mathbb{R}^{2 m}$ is defined by

$$
(\xi(p))_{i}=(\xi(p))_{i+m}:=\left|\left(p_{i}, p_{i+m}\right)^{\top}\right| \quad \text { for } p \in \mathbb{R}^{2 m}, i=1, \ldots, m .
$$

System (4.1) can also be written as the following operator equation:

$$
F\left(y^{h}, q^{h}\right):=\left[\begin{array}{c}
A_{\mu}^{h} y^{h}+B^{h} q^{h}-f^{h} \\
\max \left(g e^{h}, \gamma \xi\left(\nabla^{h} y^{h}\right)\right) \star q^{h}-\gamma g \nabla^{h} y^{h}
\end{array}\right]=0 .
$$

It is well known (see e.g. $[17,27]$ ) that the max-operator and the norm function $\xi$ involved in $(4.3)$ are semismooth. Furthermore, this is also true for the composition of semismooth functions that arises in (4.3). A particular 
element of the generalized Jacobian of $\max (0, \cdot): \mathbb{R}^{N} \rightarrow \mathbb{R}^{N}$ is the diagonal matrix $G_{\max } \in \mathbb{R}^{N \times N}$ defined by

$$
\left(G_{\max }(v)\right)_{i i}:=\left\{\begin{array}{lll}
1 & \text { if } \quad v_{i} \geq 0, \\
0 & \text { if } \quad v_{i}<0,
\end{array} \text { for } 1 \leq i \leq N .\right.
$$

Consequently, given approximations $y_{k}^{h}$ and $q_{k}^{h}$ the Newton step to (4.3) at $\left(y_{k}^{h}, q_{k}^{h}\right)$ is given by:

$$
\left[\begin{array}{cc}
A_{\mu}^{h} & B^{h} \\
\gamma\left(\chi_{\mathcal{A}_{k+1}} D\left(q_{k}^{h}\right) P^{h}\left(\nabla^{h} y_{k}^{h}\right)-g I_{2 l}\right) \nabla^{h} & D\left(m_{k}^{h}\right)
\end{array}\right]\left[\begin{array}{c}
\delta_{y} \\
\delta_{q}
\end{array}\right]=\left[\begin{array}{c}
-A_{\mu}^{h} y_{k}^{h}-B^{h} q_{k}^{h}+f^{h} \\
-D\left(m_{k}^{h}\right) q_{k}^{h}+\gamma g \nabla^{h} y_{k}^{h}
\end{array}\right],
$$

where $m_{k}^{h}:=\max \left(g e^{h}, \gamma \xi\left(\nabla^{h} y_{k}^{h}\right)\right) \in \mathbb{R}^{2 m}$, and $\chi_{\mathcal{A}}=D\left(t_{k}^{h}\right) \in \mathbb{R}^{2 m \times 2 m}$ with

$$
\left(t_{k}^{h}\right)_{i}:=\left\{\begin{array}{ll}
1 & \text { if } \\
0 & \text { else. }
\end{array} \quad \xi\left(\nabla^{h} y_{k}^{h}\right)_{i} \geq \frac{g}{\gamma}\right.
$$

Further, $P^{h} \in \mathbb{R}^{2 m \times 2 m}$ denotes the generalized Jacobian of $\xi$, i.e., for $p \in \mathbb{R}^{2 m}$, we have that

$$
P^{h}(p):=\left[\begin{array}{cc}
\frac{\partial \xi_{i}}{\partial p_{j}} & \frac{\partial \xi_{i}}{\partial p_{j+m}} \\
\frac{\partial \xi_{i+m}}{\partial p_{j}} & \frac{\partial \xi_{i+m}}{\partial p_{j+m}}
\end{array}\right]
$$

where the block diagonal matrices are defined by

$$
\begin{gathered}
\frac{\partial \xi_{i}}{\partial p_{j}}=\frac{\partial \xi_{i+m}}{\partial p_{j}}:=\delta_{i j}\left\{\begin{array}{ll}
\frac{p_{i}}{\left(p_{i}, p_{i+m}\right)^{\top} T} & \text { if }\left(p_{i}, p_{i+m}\right)^{\top} \neq 0 \\
\varepsilon_{1} & \text { if }\left(p_{i}, p_{i+m}\right)^{\top}=0
\end{array} \text { for } i, j=1, \ldots, m,\right. \\
\frac{\partial \xi_{i}}{\partial p_{j+m}}=\frac{\partial \xi_{i+m}}{\partial p_{j+m}}:=\delta_{i j}\left\{\begin{array}{ll}
\frac{p_{i+m}}{\mid\left(p_{i}, p_{i+m}\right)^{\top} \top} & \text { if } \quad\left(p_{i}, p_{i+m}\right)^{\top} \neq 0 \\
\varepsilon_{2} & \text { if } \quad\left(p_{i}, p_{i+m}\right)^{\top}=0
\end{array} \text { for } i, j=1, \ldots, m,\right.
\end{gathered}
$$

with $\varepsilon_{1}$ and $\varepsilon_{2}$ real numbers such that $\left|\left(\varepsilon_{1}, \varepsilon_{2}\right)^{\top}\right| \leq 1$.

From the invertibility of $D\left(m_{k}^{h}\right)$ we obtain that

$$
\delta_{q}=-q_{k}^{h}+\gamma D\left(m_{k}^{h}\right)^{-1}\left(g \nabla^{h} y_{k}^{h}+C_{k}^{h} \nabla^{h} \delta_{y}\right),
$$

where $C_{k}^{h}:=g I_{2 l}-\chi_{\mathcal{A}_{k+1}} D\left(q_{k}^{h}\right) P^{h}\left(\nabla^{h} y_{k}^{h}\right)$. Thus, the remaining equation for $\delta_{y}$ can be written as

$$
\Xi_{\gamma, k} \delta_{y}=\eta_{\gamma, k}
$$

where the matrix $\Xi_{\gamma, k}$ and the right hand side $\eta_{\gamma, k}$ are given by

$$
\begin{aligned}
\Xi_{\gamma, k} & :=A_{\mu}^{h}+\gamma B^{h} D\left(m_{k}^{h}\right)^{-1} C_{k}^{h} \nabla^{h}, \\
\eta_{\gamma, k} & :=-A_{\mu}^{h} y_{k}^{h}+M^{h} f^{h}-\gamma g B^{h} D\left(m_{k}^{h}\right)^{-1} \nabla^{h} y_{k}^{h} .
\end{aligned}
$$

It can be verified ( $c f .[16]$, p. 8 ) that the matrix $\Xi_{\gamma, k}$ is symmetric at the solution.

Thanks to [16], Lemma 3.3, we know that the condition $\xi\left(q_{k}^{h}\right)_{i} \leq g$, for $i=1, \ldots, m$, must hold to guarantee the positive definiteness of the matrix $C_{h}^{k}$. Moreover, we can assert that if the last condition is fulfilled, the matrix $\Xi_{\gamma, k}$ is positive definite, $\lambda_{\min }\left(\Xi_{\gamma, k}\right) \geq \lambda_{\min }\left(A_{\mu}^{h}\right)>0$ and the sequence $\left\{\Xi_{\gamma, k}^{-1}\right\}_{k \in \mathbb{N}}$ is uniformly bounded. 
Due to these results, we know that if $\xi\left(q_{k}^{h}\right)_{i} \leq g$ holds for all $i=1, \ldots, m$, the solution of (4.8) exists for all $k$ and it is a descent direction for the objective functional in $\left(\mathcal{P}_{\gamma}\right)$. However, this condition is unlikely to be fulfilled by all $1 \leq i \leq m$ and $k \in \mathbb{N}$. To overcome this difficulty, Hintermüller and Stadler [16] constructed a globalized semismooth Newton algorithm by modifying the term involving $D\left(q_{k}^{h}\right) P^{h}\left(\nabla^{y} y_{k}^{h}\right)$ for indices $i$ in which $\xi\left(q_{k}^{h}\right)_{i}>g$. This is done by replacing $q_{k}^{h}$ by

$$
g \max \left(g, \xi\left(q_{k}^{h}\right)_{i}\right)^{-1}\left(\left(q_{k}^{h}\right)_{i},\left(q_{k}^{h}\right)_{i+m}\right),
$$

when assembling the system matrix $\Xi_{\gamma, k}$. Thus, we guarantee that $\xi\left(q_{k}^{h}\right)_{i} \leq g$ for $i=1, \ldots, m$. Further, we obtain a modified system matrix, denoted by $\Xi_{\gamma, k}^{+}$, which replaces $\Xi_{\gamma, k}$ in (4.8). This new matrix is positive definite for all $\gamma$ and the sequence $\left\{\left(\Xi_{\gamma, k}^{+}\right)^{-1}\right\}_{k \in \mathbb{N}}$ is uniformly bounded.

\section{Algorithm SSN.}

1. Initialize $\left(y_{0}^{h}, q_{0}^{h}\right) \in \mathbb{R}^{m} \times \mathbb{R}^{2 m}$ and set $k=0$.

2. Estimate the active sets, i.e., determine $\chi_{\mathcal{A}_{k+1}} \in \mathbb{R}^{2 m \times 2 m}$.

3. Compute $\Xi_{\gamma, k}^{+}$if the dual variable is not feasible for all $i=1, \ldots, m$; otherwise set $\Xi_{\gamma, k}^{+}=\Xi_{\gamma, k}$. Solve

$$
\Xi_{\gamma, k}^{+} \delta_{y}=\eta_{\gamma, k}
$$

4. Compute $\delta_{q}$ from (4.7).

5. Update $y_{k+1}^{h}:=y_{k}^{h}+\delta_{y}$ and $q_{k+1}^{h}:=q_{k}^{h}+\delta_{q}$.

6. Stop, or set $k:=k+1$ and go to step 2 .

Following [16], Lemma 3.5, we know that $q_{k}^{h} \rightarrow q^{h}$ and $y_{k}^{h} \rightarrow y^{h}$ implies that $\Xi_{\gamma, k}^{+}$converges to $\Xi_{\gamma, k}$ as $k \rightarrow \infty$. Thus, thanks to this result we can state the following theorem.

Theorem 4.1. The iterates $\left(y_{k}^{h}, q_{k}^{h}\right)$ of Algorithm SSN converge superlinearly to $\left(y_{\gamma}^{h}, q_{\gamma}^{h}\right)$ provided that $\left(y_{0}^{h}, q_{0}^{h}\right)$ is sufficiently close to $\left(y_{\gamma}^{h}, q_{\gamma}^{h}\right)$.

Proof. We refer the readers to [16], Theorem 3.6, for the complete proof.

The projection procedure, which yields the matrix $\Xi_{\gamma, k}^{+}$, assures that in each iteration of Algorithm SSN, $\delta_{y}=\left(\Xi_{\gamma, k}^{+}\right)^{-1} \eta_{\gamma, k}$ constitutes a descent direction for the objective functional in $\left(\mathcal{P}_{\gamma}\right)$. Additionally, steps 3. and 4. of the algorithm involve a decoupled system of equations for $\delta_{y}$ and $\delta_{q}$, which is obtained directly, due to the regularization proposed and the structure of the method. Moreover, the computation of $\delta_{q}$ through (4.7) turns out to be computationally efficient, since only the inverse of a diagonal matrix is needed.

\section{NUMERICAL RESULTS}

In this section we present numerical experiments which illustrate the main properties of the path-following and the semismooth Newton methods applied to the numerical solution of laminar Bingham fluids. The experiments have been carried out for a constant function $f$, representing the linear decay of pressure in the pipe.

The parameter $\gamma$ is updated using the path-following strategy defined in Section 3.3. Unless we specify the contrary, we stop the Algorithm PF as soon as $r_{k}^{h}:=\left|\left(r_{k}^{1, h}, r_{k}^{2, h}, r_{k}^{3, h}\right)^{\top}\right|$ is of the order $10^{-7}$, where

$$
\begin{aligned}
r_{k}^{1, h} & =\left\|y_{\gamma_{k}}^{h}+\left(A_{\mu}^{h}\right)^{-1}\left(B^{h} q_{\gamma_{k}}^{h}-f^{h}\right)\right\|_{H_{0}^{1, h}} /\left\|f^{h}\right\|_{L^{2, h}} \\
r_{k}^{2, h} & =\left\|\max \left(g e^{h}, \xi\left(q_{\gamma_{k}}^{h}+\nabla^{h} y_{\gamma_{k}}^{h}\right)\right) \star q_{\gamma_{k}}^{h}-g\left(q_{\gamma_{k}}^{h}+\nabla^{h} y_{\gamma_{k}}^{h}\right)\right\|_{\mathbf{L}^{2, h}} \\
r_{k}^{3, h} & =\left\|\max \left(0,\left|q_{\gamma_{k}}^{h}\right|-g\right)\right\|_{L^{2, h}}
\end{aligned}
$$



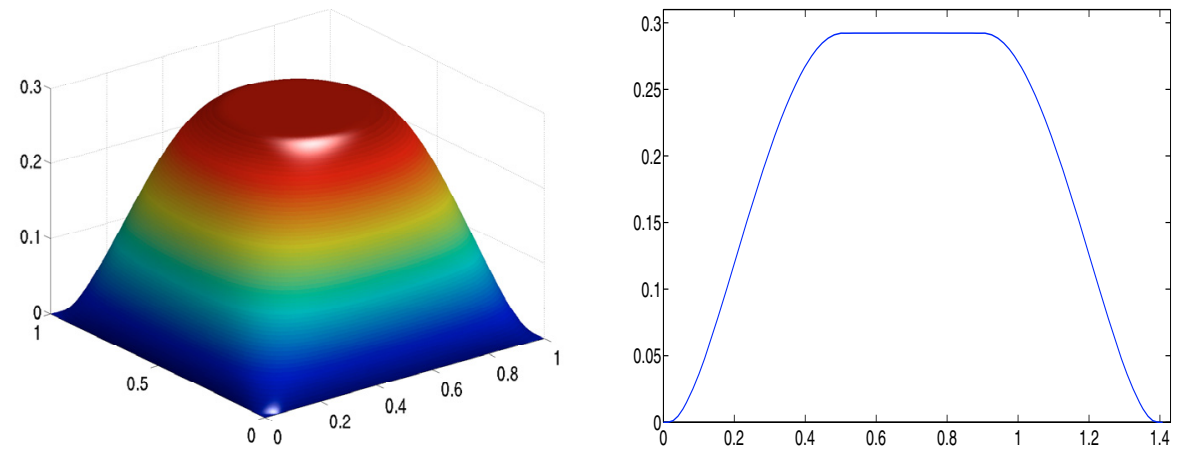

Figure 1. Example 1: flow of a Bingham fluid defined by $g=1, \mu=1$ and $f=10$ (left) and velocity profile along the diagonal $y\left(x_{1}, x_{1}\right)$ (right).

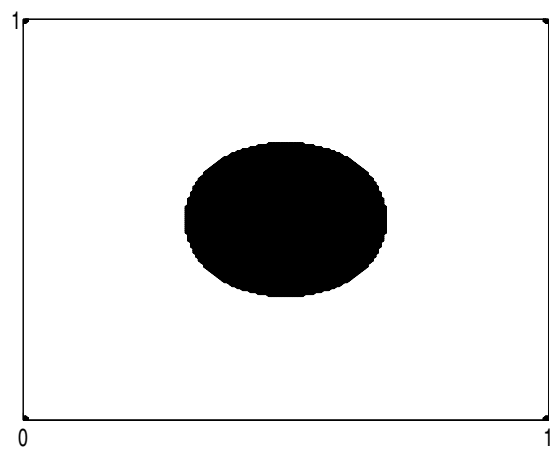

Figure 2. Example 1: final inactive set $\mathcal{I}_{\gamma}$.

with $A_{\mu}^{h}, B^{h}, \nabla^{h}$ and $\xi$ defined as in (4.1). Here $\|\cdot\|_{H_{0}^{1, h}},\|\cdot\|_{\mathbf{L}^{2, h}}$ and $\|\cdot\|_{L^{2, h}}$ denote the discrete versions of $\|\cdot\|_{H_{0}^{1}}$, $\|\cdot\|_{\mathbf{L}^{2}}$ and $\|\cdot\|_{L^{2}}$ respectively. $r_{k}^{1, h}$ and $r_{k}^{2, h}$ describe the improvement of the algorithm towards the solution of the discrete version of the optimality system $(\mathcal{S})$, while $r_{k}^{3, h}$ measures the feasibility of $q_{\gamma_{k}}^{h}$.

We use the mass matrix to calculate the integrals related to the space $V_{h}$ and a composite trapezoidal formula for the integrals associated to the space $W_{h}$. Additionally we use the sequence $\tau_{k}=0.01^{k+1}$.

\subsection{Example 1}

In our first example, we focus on the behavior of Algorithm PF. We consider $\Omega:=] 0,1[\times] 0,1[$ and compute the flow of a Bingham fluid defined by $\mu=g=1$ and $f=10$. We work with a uniform triangulation, with $h=0.0046(\approx 1 / 218)$, where $h$ is the radius of the inscribed circumferences of the triangles in the mesh. In this example, we use the initial values $\gamma_{r}=1$ and $\gamma_{0}=10$. The inner Algorithm SSN for $\gamma=\gamma_{0}$ is initialized with the solution of the Poisson problem $A_{\mu}^{h} y_{0}^{h}=f^{h}$ together with $q_{0}^{h}=\mathbf{0}$ and is finished if the residual $\delta_{y}$ is lower than $\sqrt{\epsilon}$, where $\epsilon$ denotes the machine accuracy.

The resulting velocity function is displayed in Figure 1 and the final inactive set in Figure 2. The value of the regularization parameter $\gamma_{k}$ reaches a factor of $10^{13}$ in three iterations and we obtain a maximum velocity of 0.291. The graphics illustrate the expected mechanical properties of the material, i.e., since the shear stress transmitted by a fluid layer decreases toward the center of the pipe, the Bingham fluid moves like a solid in that sector. Besides that, Figure 1 shows that there are no stagnant zones in the flow (see [22]). 
TABLe 1. $\gamma$-updates and convergence behavior of Algorithm PF for a Bingham fluid defined by $g=1, \mu=1$ and $f=10$.

\begin{tabular}{|c|c|c|c|c|}
\hline \# it. & $\gamma_{k}$ & $r_{k}^{h}$ & $\left\|y_{\gamma_{k+1}}^{h}-y_{\gamma_{k}}^{h}\right\|_{H_{0}^{1, h}}$ & $\nu_{k}^{h}$ \\
\hline 1 & $1.0081 \mathrm{e}+3$ & $1.7238 \mathrm{e}-4$ & 0.0225 & 0.0225 \\
2 & $1.0082 \mathrm{e}+7$ & $1.4855 \mathrm{e}-4$ & $2.2734 \mathrm{e}-4$ & 0.0101 \\
3 & $1.0091 \mathrm{e}+13$ & $8.7904 \mathrm{e}-7$ & $1.7390 \mathrm{e}-7$ & $7.6495 \mathrm{e}-4$ \\
\hline
\end{tabular}

TABLE 2. Number of iterations of Algorithm SSN in each path-following iteration.

\begin{tabular}{|c|c|c|c|l|}
\hline$\gamma_{k}$ & $1.0081 \mathrm{e}+3$ & $1.0082 \mathrm{e}+7$ & $1.0091 \mathrm{e}+13$ & \\
\hline$\#$ it. & 7 & 6 & 1 & $\sum=14$ \\
\hline
\end{tabular}

TABLE 3. Number of iterations of Algorithm SSN without any automatic updating strategy.

\begin{tabular}{|c|c|c|c|}
\hline$\gamma_{k}$ & $1.0081 \mathrm{e}+3$ & $1.0082 \mathrm{e}+7$ & $1.0091 \mathrm{e}+13$ \\
\hline$\#$ it. SSN & 13 & 33 & fails to converge \\
\hline
\end{tabular}

In Table 1 we report the values of the regularization parameter $\gamma_{k}$ and the residuals $r_{k}^{h}$ and

$$
\nu_{k}^{h}=\frac{\left\|y_{\gamma_{k+1}}^{h}-y_{\gamma_{k}}^{h}\right\|_{H_{0}^{1, h}}}{\left\|y_{\gamma_{k}}^{h}-y_{\gamma_{k-1}}^{h}\right\|_{H_{0}^{1, h}}}
$$

From the behavior of $r_{k}^{h}$, it is possible to observe a superlinear convergence rate of the Algorithm $\mathbf{P F}$ according to the strategy proposed in (3.61). Furthermore, the behavior of $\nu_{k}^{h}$ implies a superlinear convergence rate of $y_{\gamma_{k}}$ towards the solution, as $\gamma_{k}$ increases. These data are depicted in Figure 3, where the two magnitudes are plotted in a logarithmic scale.

In Table 2, we show the number of inner iterations that Algorithm SSN needs to achieve convergence in each iteration of Algorithm PF and the total number of SSN iterations needed. It can be observed that the path following strategy allows to reach large values of $\gamma_{k}$ and, consequently, to obtain a better approximation of the solution of the problem. In contrast to these results, in Table 3 we show the number of iterations that Algorithm SSN needs to achieve convergence without any updating strategy. In this case, the algorithm does not only need more iterations for each value of $\gamma_{k}$, but also fails to converge for large values of it.

Finally, in Figure 4 we plot and compare the path value functional $V(\gamma)$ (solid line) and the model functions $m\left(\gamma_{k}\right)$ calculated from the values $C_{1, k}, C_{2, k}$ and $G_{k}$ given in each iteration of the algorithm. It can be observed that as $\gamma_{k}$ increases, $m\left(\gamma_{k}\right)$ becomes a better model for $V(\gamma)$. However, even for small values of $\gamma_{k}$, the model functions stay close to the value functional.

\subsection{Example 2}

In this example, we compare the numerical behavior of Algorithm PF versus a penalty-Newton-Uzawaconjugate gradient method proposed by Dean et al. in [6].

We consider the flow of a Bingham fluid in the cross section of a cylindrical pipe, given by the disk defined by $\Omega:=\left\{x=\left(x_{1}, x_{2}\right) \in \mathbb{R}^{2}: x_{1}^{2}+x_{2}^{2}<R^{2}\right\}$, where $R>0$. It is well known (see [12], Ex. 2, p. 81) that in 

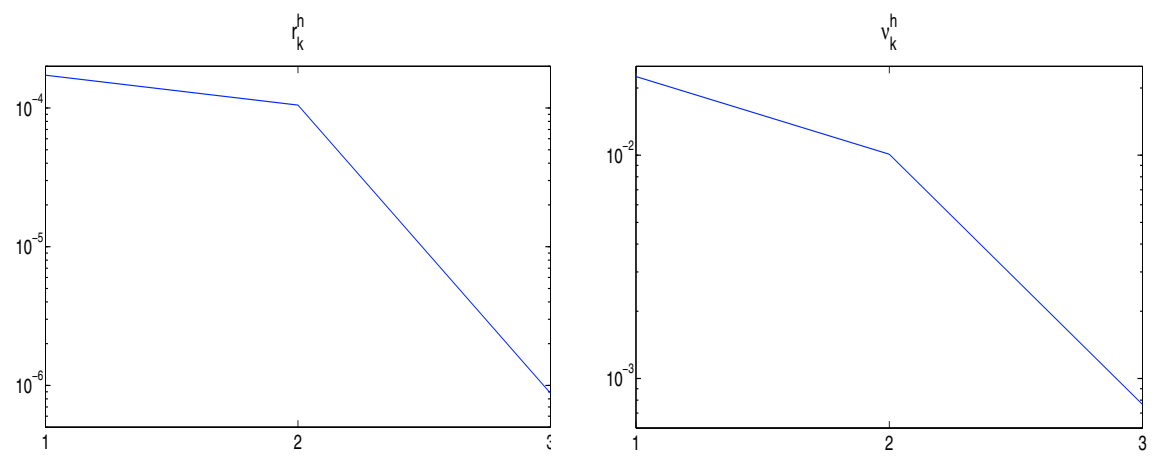

FigURE 3. Residual $r_{k}^{h}$ (left) and convergence rate $\nu_{k}^{h}$ (right).

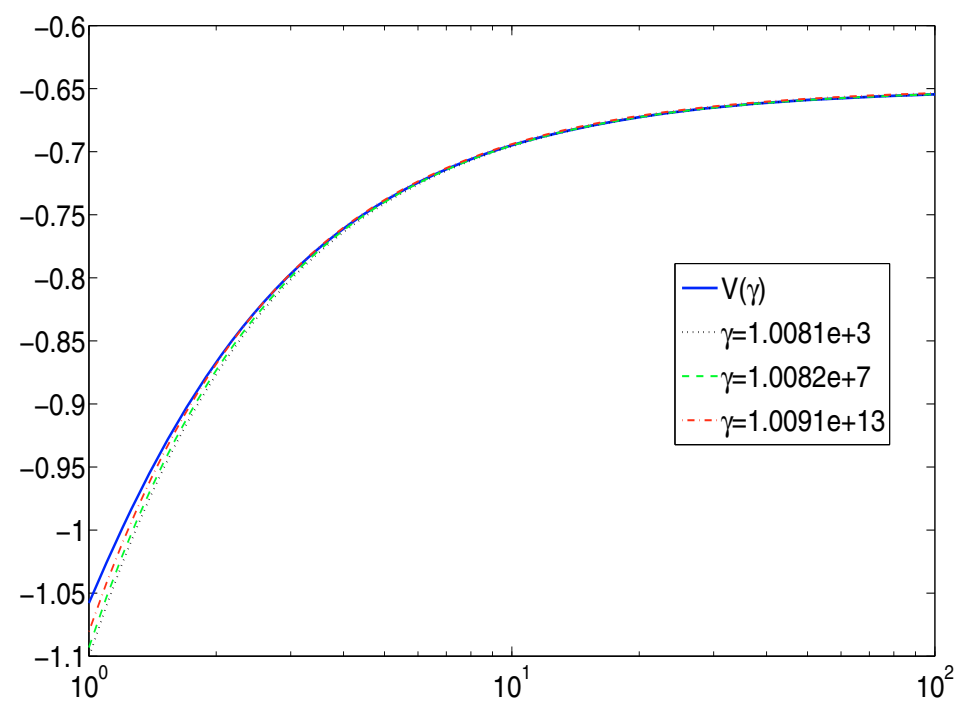

Figure 4. Primal-dual value functional (solid) versus the generated models $m(\gamma)$ given by Algorithm PF.

this case, the exact solution of the problem is given by

$$
\bar{y}(x)= \begin{cases}\left(\frac{R-\sqrt{x_{1}^{2}+x_{2}^{2}}}{2 \mu}\right)\left[\frac{f}{2}\left(R+\sqrt{x_{1}^{2}+x_{2}^{2}}\right)-2 g\right] & \text { if } \quad \frac{2 g}{f} \leq \sqrt{x_{1}^{2}+x_{2}^{2}} \leq R \\ \left(\frac{R-\frac{2 g}{f}}{2 \mu}\right)\left[\frac{f}{2}\left(R+\frac{2 g}{f}\right)-2 g\right] & \text { if } \quad 0 \leq \sqrt{x_{1}^{2}+x_{2}^{2}} \leq \frac{2 g}{f}\end{cases}
$$

We take the parameter values $R=1 / 4, f=16$ and $\mu=1 / 4$ and consider a non-uniform grid, with mesh size $h=0.0043(\approx 1 / 233)$, where $h$ is the maximum radius of the circumferences inscribed in the triangles of the mesh.

Next, we shortly describe the penalty-Newton-Uzawa-conjugate gradient method proposed in [6], Section 7.5, and hereafter referred to as pNUz. The method is based on a $C^{2}$ regularization of the dual problem (see [6], Eq. (100)). By applying a Newton method to the resulting regularized primal dual system (cf. [6], Eq. (102)), the authors obtain the following coupled system of equations for the increments $\delta_{y}$ and $\delta_{q}$ in each Newton 
TABLE 4. Numerical behavior of the algorithms $\mathbf{P F}$ and $\mathbf{p N U z}$, for varying values of $g$.

\begin{tabular}{|c||c|c|c|c||c|c|c|c|}
\hline$g$ & $\gamma$ & \# PF & \# SSN & l.s. & $\varepsilon$ & \# pN & \# Uz & l.s. \\
\hline 0.2 & 333.3654 & 1 & 6 & 6 & $2.9997 \mathrm{e}-3$ & 16 & 116 & 143 \\
& $3.7041 \mathrm{e}+5$ & 2 & 10 & 10 & $2.6997 \mathrm{e}-6$ & 21 & 140 & 182 \\
& $1.3719 \mathrm{e}+10$ & 3 & 11 & 11 & $7.2892 \mathrm{e}-11$ & 25 & 159 & 209 \\
1 & 667.6761 & 1 & 7 & 7 & $1.4977 \mathrm{e}-3$ & 8 & 50 & 66 \\
& $7.4189 \mathrm{e}+5$ & 2 & 12 & 12 & $1.3479 \mathrm{e}-6$ & 14 & 81 & 109 \\
& $3.4335 \mathrm{e}+10$ & 3 & 15 & 15 & $2.9125 \mathrm{e}-11$ & 19 & 101 & 134 \\
1.5 & 670.0717 & 1 & 8 & 8 & $1.4924 \mathrm{e}-3$ & 6 & 32 & 44 \\
& $7.4462 \mathrm{e}+5$ & 2 & 12 & 12 & $1.3430 \mathrm{e}-6$ & 11 & 57 & 79 \\
& $2.7579 \mathrm{e}+10$ & 3 & 14 & 14 & $3.6259 \mathrm{e}-11$ & 17 & 82 & 116 \\
\hline
\end{tabular}

iteration:

$$
\begin{aligned}
& -\mu \Delta \delta_{y}=g \operatorname{div} \delta_{q} \text { in } \Omega, \delta_{y}=0 \text { on } \Gamma \\
& -\varepsilon \nabla \delta_{y}+\left(\left|q_{k}^{h}\right|^{2}-1\right)^{+2} \delta_{q}+4\left(\left|q_{k}^{h}\right|^{2}-1\right)^{+} q_{k}^{h}\left\langle q_{k}^{h}, \delta_{q}\right\rangle=\varepsilon \nabla y_{k}-\left(\left|q_{k}^{h}\right|^{2}-1\right)^{+2} q_{k}^{h},
\end{aligned}
$$

where $y_{k}^{h}$ and $q_{k}^{h}$ are the iterates of the Newton method and $z^{+}:=\max (0, z)$. To uncouple system (5.2) the authors propose an Uzawa-conjugate gradient algorithm, which requires, in each of its iterations, the solution of an additional Dirichlet problem [6], p. 50. For the full description of the algorithm we refer to [6]. Hereafter, we refer to the outer Newton algorithm as Algorithm $\mathbf{p N}$ and to the inner Uzawa-conjugate gradient algorithm as Algorithm Uz.

Initialization and stopping criteria. We initialize Algorithm $\mathbf{P F}$ with $\gamma_{r}=1$ and $\gamma_{0}=10$. In each $\mathbf{P F}$ iteration the inner Algorithm SSN stops as soon as $\left\|\delta_{y}\right\|_{H_{0}^{1, h}}$ is of the order $10^{-6}$. Further, we modify the tolerance of $\mathbf{P F}$ in order to finish with different and increasing values of $\gamma_{k}$.

For the initialization of $\mathbf{p N U z}$ we follow [6]. We initialize the Algorithm $\mathbf{p N}$ with $q_{0}^{h}=\mathbf{0}$ and with the solution of $A_{\mu}^{h} y_{0}^{h}+B^{h} q_{0}^{h}=f^{h}$. The Algorithm $\mathbf{U z}$ is initialized with the solution of the following system

$$
\begin{aligned}
a\left(\nabla \delta_{y}^{0}, \nabla v\right)= & -g\left(\delta_{q}^{0}, \nabla v\right)_{\mathbf{L}^{2}(\Omega)}, \quad \text { for all } v \in H_{0}^{1}(\Omega), \\
\left(\mathbf{g}^{0}, p\right)_{\mathbf{L}^{2}(\Omega)}= & -\varepsilon\left(\nabla\left(\delta_{y}^{0}+y_{k}^{h}\right), p\right)_{\mathbf{L}^{2}(\Omega)}+\left(\left(\left|q_{k}^{h}\right|^{2}-1\right)^{+2}\left(\delta_{q}^{0}+q_{k}^{h}\right), p\right)_{\mathbf{L}^{2}(\Omega)} \\
& +4\left(\left(\left|q_{k}^{h}\right|^{2}-1\right)^{+}\left\langle q_{k}^{h}, \delta_{q}^{0}\right\rangle q_{k}^{h}, p\right)_{\mathbf{L}^{2}(\Omega)}, \quad \text { for all } p \in \mathbf{L}^{2}(\Omega) .
\end{aligned}
$$

We stop the Algorithm $\mathbf{p N}$ as soon as $\left\|\delta_{\mathbf{q}}\right\|_{H_{0}^{1, h}}$ is of the order $10^{-6}$ and the Algorithm $\mathbf{U z}$ as soon as $\int_{\Omega}\left|\mathbf{g}^{m+1}\right|^{2} \mathrm{~d} x / \int_{\Omega}\left|\mathbf{g}^{0}\right|^{2} \mathrm{~d} x$ is of the order $10^{-4}$.

Numerical behavior. Here, we compare the behavior of $(\mathbf{P F})$ and $(\mathbf{p N U z})$ for different values of $g$ and of the regularization parameters. To put both methods into correspondence and compare their performance, we set the parameter value $\varepsilon=1 / \gamma$ and use this value in Algorithm $\mathbf{p N}$.

In Table 4, we show the total number of iterations of Algorithms $\mathbf{P F}$ and $\mathbf{p N}$ and also the total number of SSN and $\mathbf{U z}$ iterations needed within the $\mathbf{P F}$ and $\mathbf{p N}$ methods, respectively. The total number of linear systems solved within the $\mathbf{P F}$ and $\mathbf{p N}$ Algorithms are shown in the fourth and last columns, respectively.

From the data in Table 4 it can be observed that there exists a significant difference with respect to the number of linear systems solved within the $\mathbf{P F}$ and the $\mathbf{p N U z}$ methods. This fact can be explained from the different structure of both methods. On the one hand, the proposed Tikhonov regularization together with the semismooth Newton method lead directly to a decoupled primal dual system to be solved in each Newton type iteration (see Eq. (4.5)). Consequently, Algorithm SSN only needs to solve one $n \times n$ linear system per iteration (see Eq. (4.8)) and, therefore, the total number of linear systems to be solved within PF coincide with the total number of iterations of the inner Algorithm SSN. 
TABLE 5. Numerical behavior of the Algorithm PF for varying values of $g$.

\begin{tabular}{|c||c|c|c||c|c|c|}
\hline$g$ & $\gamma$ & $\left\|y_{\gamma}^{h}-\bar{y}\right\|_{L^{2, h}}$ & $\left\|y_{\gamma}^{h}-\bar{y}\right\|_{H_{0}^{1, h}}$ & $\varepsilon$ & $\left\|y_{\varepsilon}^{h}-\bar{y}\right\|_{L^{2, h}}$ & $\left\|y_{\varepsilon}^{h}-\bar{y}\right\|_{H_{0}^{1, h}}$ \\
\hline 0.2 & 333.36 & $1.0125 \mathrm{e}-4$ & 0.0025 & $2.99 \mathrm{e}-3$ & 0.0019 & 0.0188 \\
& $3.70 \mathrm{e}+5$ & $9.9308 \mathrm{e}-5$ & 0.0015 & $2.69 \mathrm{e}-6$ & $3.8455 \mathrm{e}-3$ & 0.0017 \\
& $1.37 \mathrm{e}+10$ & $9.9284 \mathrm{e}-5$ & 0.0015 & $7.28 \mathrm{e}-11$ & $9.9730 \mathrm{e}-5$ & 0.0014 \\
1 & 667.67 & $3.2885 \mathrm{e}-4$ & 0.0086 & $1.49 \mathrm{e}-3$ & 0.0050 & 0.0539 \\
& $7.41 \mathrm{e}+5$ & $8.2123 \mathrm{e}-5$ & 0.0043 & $1.34 \mathrm{e}-6$ & $1.7447 \mathrm{e}-4$ & 0.0042 \\
& $3.43 \mathrm{e}+10$ & $8.2036 \mathrm{e}-5$ & 0.0043 & $2.91 \mathrm{e}-11$ & $8.4041 \mathrm{e}-5$ & 0.0041 \\
1.5 & 670.07 & 0.0012 & 0.0170 & $1.49 \mathrm{e}-3$ & 0.0031 & 0.0413 \\
& $7.44 \mathrm{e}+5$ & $5.7134 \mathrm{e}-5$ & 0.0020 & $1.34 \mathrm{e}-6$ & $1.2055 \mathrm{e}-4$ & 0.0021 \\
& $2.75 \mathrm{e}+10$ & $3.4947 \mathrm{e}-5$ & 0.0020 & $3.62 \mathrm{e}-11$ & $2.8791 \mathrm{e}-5$ & 0.0017 \\
\hline
\end{tabular}

On the other hand, the regularization proposed in [6] leads to the coupled system (5.2). By using an Uzawaconjugate gradient method the authors manage to decouple this system. However, Algorithm Uz needs to solve at least one $n \times n$ linear system per iteration and two additional $n \times n$ linear systems every time it is called by Algorithm pN. Therefore, a larger number of linear systems to be solved is expected.

From Table 4, comparing the total number of Newton type iterations needed by both methods (\# SSN and \# pN, respectively), a better behavior of $\mathbf{P F}$ can be observed. The difference is, however, not significant. Moreover, the number of iterations increases as $\gamma$ increases and $\varepsilon$ decreases, respectively.

Approximation errors. In Table 5, we show the approximation errors of the $\mathbf{P F}$ and the $\mathbf{p N U z}$ methods, where $\bar{y}$ is given by (5.1). We can see that the two methods reach competitive values for the approximation errors in the $L^{2}(\Omega)$ and $H_{0}^{1}(\Omega)$ norms.

\subsection{Conclusions}

- The proposed path following strategy allows to reach large values of the regularization parameter $\gamma$ and, therefore, get better approximate solutions. Comparing with the application of the semismooth Newton algorithm without updating strategy, larger values of $\gamma$ can be obtained and less SSN-iterations are needed (see Tabs. 2 and 3).

- The path following strategy together with the semismooth Newton method lead directly to the decoupled system (4.5) to be solved in each Newton type iteration. On the contrary, in pNUz an additional Uzawaconjugate gradient method is used to get a decoupled system. As a consequence, the number of $n \times n$ linear systems to be solved within the $\mathbf{P F}$ Algorithm is smaller than the number of linear systems to be solved within $\mathbf{p N U z}$ (see Tab. 4).

- From the exhibited properties of the path following strategy, we think that promising results can be expected from its application to more challenging problems. In particular we intend to consider in the near future the application of the method to other variational inequalities of the second kind.

\section{A. Appendix}

Proof of Proposition 3.5. From $\left(\mathcal{S}_{\gamma}\right)$ we get that

$$
a\left(y_{\bar{\gamma}}-y_{\gamma}, v\right)+g\left(\frac{\bar{\gamma} \nabla y_{\bar{\gamma}}}{\theta_{\bar{\gamma}}}-\frac{\gamma \nabla y_{\gamma}}{\theta_{\gamma}}, \nabla v\right)_{\mathbf{L}^{2}(\Omega)}=0, \text { for all } v \in H_{0}^{1}(\Omega)
$$

with $\theta_{\gamma}(x):=\max \left(g, \gamma\left|\nabla y_{\gamma}(x)\right|\right)$. First, note that, since $\dot{y}_{\gamma}^{+}$is an accumulation point in $H_{0}^{1}(\Omega)$, we can easily obtain that

$$
\lim _{\bar{\gamma} \downarrow \gamma}(\bar{\gamma}-\gamma)^{-1} a\left(y_{\bar{\gamma}}-y_{\gamma}, v\right)=a\left(\dot{y}_{\gamma}^{+}, v\right)
$$


Now, we analyze the second term on the left hand side of (A.1). Let us introduce the following notation

$$
\mathrm{I}_{j}=g\left(\frac{\bar{\gamma} \nabla y_{\bar{\gamma}}}{\theta_{\bar{\gamma}}}-\frac{\gamma \nabla y_{\gamma}}{\theta_{\gamma}}, \nabla v\right)_{\mathbf{L}^{2}\left(\Omega_{j}\right)}, j=1, \ldots, 4
$$

where $\Omega_{1}:=\mathcal{A}_{\bar{\gamma}} \cap \mathcal{A}_{\gamma}, \Omega_{2}:=\mathcal{A}_{\bar{\gamma}} \cap \mathcal{I}_{\gamma}, \Omega_{3}:=\mathcal{A}_{\gamma} \cap \mathcal{I}_{\bar{\gamma}}$, and $\Omega_{4}:=\mathcal{I}_{\gamma} \cap \mathcal{I}_{\bar{\gamma}}$ give a disjoint partitioning of $\Omega$. Now, we analyze the integrals $\mathrm{I}_{j}$ separately.

On $\Omega_{1}\left(\lim _{\bar{\gamma} \downarrow \gamma}(\bar{\gamma}-\gamma)^{-1} \mathrm{I}_{1}\right)$ : We recall that $\theta_{\bar{\gamma}}(x)=\bar{\gamma}\left|\nabla y_{\bar{\gamma}}(x)\right|$ and $\theta_{\gamma}(x)=\gamma\left|\nabla y_{\gamma}(x)\right|$, a.e. in $\Omega_{1}$. Thus, we have the following pointwise equality

$$
\begin{aligned}
\frac{\bar{\gamma} \nabla y_{\bar{\gamma}}(x)}{\theta_{\bar{\gamma}}(x)}-\frac{\gamma \nabla y_{\gamma}(x)}{\theta_{\gamma}(x)} & =\frac{\nabla y_{\bar{\gamma}}(x)}{\left|\nabla y_{\bar{\gamma}}(x)\right|}-\frac{\nabla y_{\gamma}(x)}{\left|\nabla y_{\gamma}(x)\right|} \\
& =\frac{\nabla\left(y_{\bar{\gamma}}(x)-y_{\gamma}(x)\right)}{\left|\nabla y_{\gamma}(x)\right|}-\frac{\left(\left|\nabla y_{\bar{\gamma}}(x)\right|-\left|\nabla y_{\gamma}(x)\right|\right) \nabla y_{\bar{\gamma}}(x)}{\left|\nabla y_{\bar{\gamma}}(x)\right|\left|\nabla y_{\gamma}(x)\right|},
\end{aligned}
$$

which implies that

$$
(\bar{\gamma}-\gamma)^{-1} \mathrm{I}_{1}=g \underbrace{\int_{\Omega_{1}}\left\langle\frac{\nabla\left(\frac{y_{\bar{\gamma}}-y_{\gamma}}{\bar{\gamma}-\gamma}\right)}{\left|\nabla y_{\gamma}\right|}, \nabla v\right\rangle \mathrm{d} x}_{:=J_{1}}-g \underbrace{\int_{\Omega_{1}}\left\langle\frac{\left(\left|\nabla y_{\bar{\gamma}}\right|-\left|\nabla y_{\gamma}\right|\right) \nabla y_{\bar{\gamma}}}{(\bar{\gamma}-\gamma)\left|\nabla y_{\bar{\gamma}}\right|\left|\nabla y_{\gamma}\right|}, \nabla v\right\rangle \mathrm{d} x}_{:=J_{2}} .
$$

Now, we analyze $J_{1}$ and $J_{2}$ separately.

(i) $J_{1}$ : Since $\mathcal{A}_{\gamma}=\left(\mathcal{A}_{\gamma} \cap \mathcal{A}_{\bar{\gamma}}\right) \cup\left(\mathcal{A}_{\gamma} \backslash \mathcal{A}_{\bar{\gamma}}\right)$, we have that

$$
J_{1}=\int_{\mathcal{A}_{\gamma}}\left\langle\frac{1}{\left|\nabla y_{\gamma}\right|} \nabla\left(\frac{y_{\bar{\gamma}}-y_{\gamma}}{\bar{\gamma}-\gamma}\right), \nabla v\right\rangle \mathrm{d} x-\int_{\mathcal{A}_{\gamma} \backslash \mathcal{A}_{\bar{\gamma}}}\left\langle\frac{1}{\left|\nabla y_{\gamma}\right|} \nabla\left(\frac{y_{\bar{\gamma}}-y_{\gamma}}{\bar{\gamma}-\gamma}\right), \nabla v\right\rangle \mathrm{d} x
$$

Thus, since $\dot{y}_{\gamma}^{+}$is an accumulation point of $\frac{y_{\bar{\gamma}}-y_{\gamma}}{\bar{\gamma}-\gamma}$ in $H_{0}^{1}(\Omega)$ as $\bar{\gamma} \downarrow \gamma$, we obtain, for all $v \in H_{0}^{1}(\Omega)$, that

$$
\int_{\mathcal{A}_{\gamma}}\left\langle\frac{1}{\left|\nabla y_{\gamma}\right|} \nabla\left(\frac{y_{\bar{\gamma}}-y_{\gamma}}{\bar{\gamma}-\gamma}\right), \nabla v\right\rangle \mathrm{d} x \rightarrow \int_{\mathcal{A}_{\gamma}}\left\langle\frac{\nabla \dot{y}_{\gamma}^{+}}{\left|\nabla y_{\gamma}\right|}, \nabla v\right\rangle \mathrm{d} x, \text { as } \bar{\gamma} \downarrow \gamma
$$

On the other hand, we know that in $\mathcal{A}_{\gamma}$ it holds that $\frac{g}{\gamma} \leq\left|\nabla y_{\gamma}(x)\right|$. Thus, Cauchy-Schwarz and Hölder inequalities and Theorem 3.2 imply that

$$
\begin{aligned}
\int_{\mathcal{A}_{\gamma} \backslash \mathcal{A}_{\bar{\gamma}}}\left\langle\frac{1}{\left|\nabla y_{\gamma}\right|} \nabla\left(\frac{y_{\bar{\gamma}}-y_{\gamma}}{\bar{\gamma}-\gamma}\right), \nabla v\right\rangle \mathrm{d} x & \leq \frac{\gamma}{g(\bar{\gamma}-\gamma)}\left(\int_{\mathcal{A}_{\gamma} \backslash \mathcal{A}_{\bar{\gamma}}}\left|\nabla y_{\bar{\gamma}}-\nabla y_{\gamma}\right|^{2} \mathrm{~d} x\right)^{1 / 2}\|v\|_{H_{0}^{1}\left(\mathcal{A}_{\gamma} \backslash \mathcal{A}_{\bar{\gamma}}\right)} \\
& \leq \frac{\gamma}{g(\bar{\gamma}-\gamma)}\left(\int_{\Omega}\left|\nabla y_{\bar{\gamma}}-\nabla y_{\gamma}\right|^{2} \mathrm{~d} x\right)^{1 / 2}\|v\|_{H_{0}^{1}\left(\mathcal{A}_{\gamma} \backslash \mathcal{A}_{\bar{\gamma}}\right)} \\
& \leq \frac{\gamma L}{g}\|v\|_{H_{0}^{1}\left(\mathcal{A}_{\gamma} \backslash \mathcal{A}_{\bar{\gamma}}\right)},
\end{aligned}
$$

which, due to Lemma 3.4 and [2], Lemma A1.16, yields that

$$
\int_{\mathcal{A}_{\gamma} \backslash \mathcal{A}_{\bar{\gamma}}}\left\langle\frac{1}{\left|\nabla y_{\gamma}\right|} \nabla\left(\frac{y_{\bar{\gamma}}-y_{\gamma}}{\bar{\gamma}-\gamma}\right), \nabla v\right\rangle \mathrm{d} x \rightarrow 0, \text { for all } v \in H_{0}^{1}(\Omega) \text {. }
$$


Thus, (A.3) and (A.4) imply

$$
\lim _{\bar{\gamma} \downarrow \gamma} J_{1}=\int_{\mathcal{A}_{\gamma}}\left\langle\frac{\nabla \dot{y}_{\gamma}^{+}}{\left|\nabla y_{\gamma}\right|}, \nabla v\right\rangle \mathrm{d} x, \text { for } v \in H_{0}^{1}(\Omega) .
$$

(ii) $J_{2}$ : Here, we prove that

$$
\lim _{\bar{\gamma} \downarrow \gamma} J_{2}=\int_{\mathcal{A}_{\gamma}}\left\langle\frac{\left\langle\nabla y_{\gamma}, \nabla \dot{y}_{\gamma}^{+}\right\rangle}{\left|\nabla y_{\gamma}\right|^{3}} \nabla y_{\gamma}, \nabla v\right\rangle \mathrm{d} x
$$

For that purpose, we use again the disjoint partitioning $\mathcal{A}_{\gamma}=\left(\mathcal{A}_{\gamma} \cap \mathcal{A}_{\bar{\gamma}}\right) \cup\left(\mathcal{A}_{\gamma} \backslash \mathcal{A}_{\bar{\gamma}}\right)$.

First, note that $\left|\nabla y_{\gamma}(x)\right| \geq \frac{g}{\gamma}$, a.e. in $\Omega_{1}$. Thus, Hölder inequality and Theorem 3.2 imply that

$$
\begin{aligned}
\left|\int_{\mathcal{A}_{\gamma} \backslash \mathcal{A}_{\bar{\gamma}}}\left\langle\frac{\left|\nabla y_{\bar{\gamma}}\right|-\left|\nabla y_{\gamma}\right|}{(\bar{\gamma}-\gamma)\left|\nabla y_{\bar{\gamma}}\right|\left|\nabla y_{\gamma}\right|} \nabla y_{\bar{\gamma}}, \nabla v\right\rangle \mathrm{d} x\right| & \leq \int_{\mathcal{A}_{\gamma} \backslash \mathcal{A}_{\bar{\gamma}}} \frac{\left|\nabla y_{\bar{\gamma}}-\nabla y_{\gamma}\right||\nabla v|}{|\bar{\gamma}-\gamma|\left|\nabla y_{\gamma}\right|} \mathrm{d} x \\
& \leq \frac{\gamma}{g|\bar{\gamma}-\gamma|}\left\|y_{\bar{\gamma}}-y_{\gamma}\right\|_{H_{0}^{1}}\|v\|_{H_{0}^{1}\left(\mathcal{A}_{\gamma} \backslash \mathcal{A}_{\bar{\gamma}}\right)} \\
& \leq \frac{\gamma L}{g}\|v\|_{H_{0}^{1}\left(\mathcal{A}_{\gamma} \backslash \mathcal{A}_{\bar{\gamma}}\right)} .
\end{aligned}
$$

Consequently, from Lemma 3.4 and [2], Lemma A1.16, we conclude that

$$
\left|\int_{\mathcal{A}_{\gamma} \backslash \mathcal{A}_{\bar{\gamma}}}\left\langle\frac{\left|\nabla y_{\bar{\gamma}}\right|-\left|\nabla y_{\gamma}\right|}{(\bar{\gamma}-\gamma)\left|\nabla y_{\bar{\gamma}}\right|\left|\nabla y_{\gamma}\right|} \nabla y_{\bar{\gamma}}, \nabla v\right\rangle \mathrm{d} x\right| \rightarrow 0 \text {, as } \bar{\gamma} \downarrow \gamma, \text { for all } v \in H_{0}^{1}(\Omega) \text {. }
$$

On the other hand we obtain that

$$
\begin{aligned}
\left|\int_{\mathcal{A}_{\gamma}}\left\langle\frac{\left(\left|\nabla y_{\bar{\gamma}}\right|-\left|\nabla y_{\gamma}\right|\right) \nabla y_{\bar{\gamma}}}{(\bar{\gamma}-\gamma)\left|\nabla y_{\bar{\gamma}}\right|\left|\nabla y_{\gamma}\right|}, \nabla v\right\rangle \mathrm{d} x-\int_{\mathcal{A}_{\gamma}}\left\langle\frac{\left\langle\nabla y_{\gamma}, \nabla \dot{y}_{\gamma}^{+}\right\rangle}{\left|\nabla y_{\gamma}\right|^{3}} \nabla y_{\gamma}, \nabla v\right\rangle \mathrm{d} x\right| \leq \\
\left|\int_{\mathcal{A}_{\gamma}}\left\langle\left[\frac{\left|\nabla y_{\bar{\gamma}}\right|-\left|\nabla y_{\gamma}\right|}{\bar{\gamma}-\gamma}\right]\left[\frac{\nabla y_{\bar{\gamma}}}{\left|\nabla y_{\bar{\gamma}}\right|\left|\nabla y_{\gamma}\right|}-\frac{\nabla y_{\gamma}}{\left|\nabla y_{\gamma}\right|^{2}}\right], \nabla v\right\rangle \mathrm{d} x\right| \\
+\left|\int_{\mathcal{A}_{\gamma}}\left[\frac{\left|\nabla y_{\bar{\gamma}}\right|-\left|\nabla y_{\gamma}\right|}{\bar{\gamma}-\gamma}-\frac{\left\langle\nabla y_{\gamma}, \nabla \dot{y}_{\gamma}^{+}\right\rangle}{\left|\nabla y_{\gamma}\right|}\right]\left\langle\frac{\nabla y_{\gamma}}{\left|\nabla y_{\gamma}\right|^{2}}, \nabla v\right\rangle \mathrm{d} x\right| .
\end{aligned}
$$

Considering the first term of the right side of (A.8) and recalling that $\frac{g}{\gamma} \leq\left|\nabla y_{\gamma}(x)\right|$ a.e. in $\mathcal{A}_{\gamma}$, we obtain, from Theorem 3.2, Cauchy-Schwarz and Hölder inequalities, that

$$
\begin{gathered}
\int_{\mathcal{A}_{\gamma}}\left|\left\langle\left[\frac{\left|\nabla y_{\bar{\gamma}}\right|-\left|\nabla y_{\gamma}\right|}{\bar{\gamma}-\gamma}\right]\left[\frac{\nabla y_{\bar{\gamma}}}{\left|\nabla y_{\bar{\gamma}}\right|\left|\nabla y_{\gamma}\right|}-\frac{\nabla y_{\gamma}}{\left|\nabla y_{\gamma}\right|^{2}}\right], \nabla v\right\rangle\right| \mathrm{d} x \leq \int_{\mathcal{A}_{\gamma}} \frac{\gamma}{g} \frac{\left|\nabla y_{\bar{\gamma}}-\nabla y_{\gamma}\right|}{\bar{\gamma}-\gamma}\left|\left\langle\frac{\nabla y_{\bar{\gamma}}}{\left|\nabla y_{\bar{\gamma}}\right|}-\frac{\nabla y_{\gamma}}{\left|\nabla y_{\gamma}\right|}, \nabla v\right\rangle\right| \mathrm{d} x \\
\leq \frac{\gamma}{g|\bar{\gamma}-\gamma|}\left\|y_{\bar{\gamma}}-y_{\gamma}\right\|_{H_{0}^{1}}\left\|\left\langle\frac{\nabla y_{\bar{\gamma}}}{\left|\nabla y_{\bar{\gamma}}\right|}-\frac{\nabla y_{\gamma}}{\left|\nabla y_{\gamma}\right|}, \nabla v\right\rangle\right\|_{L^{2}(\Omega)} \\
\leq \frac{\gamma L}{g}\left\|\left\langle\frac{\nabla y_{\bar{\gamma}}}{\left|\nabla y_{\bar{\gamma}}\right|}-\frac{\nabla y_{\gamma}}{\left|\nabla y_{\gamma}\right|}, \nabla v\right\rangle\right\|_{L^{2}(\Omega)} .
\end{gathered}
$$

Thus, since $\nabla y_{\bar{\gamma}} \rightarrow \nabla y_{\gamma}$ strongly in $\mathbf{L}^{2}(\Omega)$, as $\bar{\gamma} \rightarrow \gamma$, we have that

$$
\int_{\mathcal{A}_{\gamma}}\left|\left\langle\frac{\nabla y_{\bar{\gamma}}}{\left|\nabla y_{\bar{\gamma}}\right|}-\frac{\nabla y_{\gamma}}{\left|\nabla y_{\gamma}\right|}, \nabla v\right\rangle\right|^{2} \mathrm{~d} x \rightarrow 0, \text { for all } v \in H_{0}^{1}(\Omega) .
$$


For the second term on the right hand side of (A.8), we have the following estimate

$$
\begin{aligned}
& \left|\int_{\mathcal{A}_{\gamma}}\left[\frac{\left|\nabla y_{\bar{\gamma}}\right|-\left|\nabla y_{\gamma}\right|}{\bar{\gamma}-\gamma}-\frac{\left\langle\nabla y_{\gamma}, \nabla \dot{y}_{\gamma}^{+}\right\rangle}{\left|\nabla y_{\gamma}\right|}\right]\left\langle\frac{\nabla y_{\gamma}}{\left|\nabla y_{\gamma}\right|^{2}}, \nabla v\right\rangle \mathrm{d} x\right| \leq \\
& \left|\int_{\mathcal{A}_{\gamma}}\left[\frac{\left\langle\nabla y_{\gamma}, \nabla \frac{y_{\bar{\gamma}}-y_{\gamma}}{\bar{\gamma}-\gamma}\right\rangle}{\left|\nabla y_{\gamma}\right|}-\frac{\left\langle\nabla y_{\gamma}, \nabla \dot{y}_{\gamma}\right\rangle}{\left|\nabla y_{\gamma}\right|}\right]\left\langle\frac{\nabla y_{\gamma}}{\left|\nabla y_{\gamma}\right|^{2}}, \nabla v\right\rangle \mathrm{d} x\right| \\
& +\left|\int_{\mathcal{A}_{\gamma}}\left[\frac{\left|\nabla y_{\bar{\gamma}}\right|-\left|\nabla y_{\gamma}\right|}{\bar{\gamma}-\gamma}-\frac{\left\langle\nabla y_{\gamma}, \nabla \frac{y_{\bar{\gamma}}-y_{\gamma}}{\bar{\gamma}-\gamma}\right\rangle}{\left|\nabla y_{\gamma}\right|}\right]\left\langle\frac{\nabla y_{\gamma}}{\left|\nabla y_{\gamma}\right|^{2}}, \nabla v\right\rangle \mathrm{d} x\right| .
\end{aligned}
$$

Now, since $\dot{y}_{\gamma}^{+}$is an accumulation point of $\nabla\left(\frac{y_{\bar{\gamma}}-y_{\gamma}}{\bar{\gamma}-\gamma}\right)$ in $H_{0}^{1}(\Omega)$ as $\bar{\gamma} \downarrow \gamma$, we have, for all $v \in H_{0}^{1}(\Omega)$, that

$$
\left|\int_{\mathcal{A}_{\gamma}}\left[\frac{\left\langle\nabla y_{\gamma}, \nabla \frac{y_{\bar{\gamma}}-y_{\gamma}}{\bar{\gamma}-\gamma}\right\rangle}{\left|\nabla y_{\gamma}\right|}-\frac{\left\langle\nabla y_{\gamma}, \nabla \dot{y}_{\gamma}\right\rangle}{\left|\nabla y_{\gamma}\right|}\right]\left\langle\frac{\nabla y_{\gamma}}{\left|\nabla y_{\gamma}\right|^{2}}, \nabla v\right\rangle \mathrm{d} x\right| \rightarrow 0 \text {, as } \bar{\gamma} \downarrow \gamma \text {. }
$$

Next, we turn to the second integral in the right hand side of (A.10). Then, from the Cauchy-Schwarz inequality, and due to the fact that $\left|\nabla y_{\gamma}(x)\right| \geq \frac{g}{\gamma}$ a.e. in $\mathcal{A}_{\gamma}$, we have that

$$
\begin{aligned}
\mid \int_{\mathcal{A}_{\gamma}} & {\left[\frac{\left|\nabla y_{\bar{\gamma}}\right|-\left|\nabla y_{\gamma}\right|}{\bar{\gamma}-\gamma}-\frac{\left\langle\nabla y_{\gamma}, \nabla\left(\frac{y_{\bar{\gamma}}-y_{\gamma}}{\bar{\gamma}-\gamma}\right)\right\rangle}{\left|\nabla y_{\gamma}\right|}\right]\left\langle\frac{\nabla y_{\gamma}}{\left|\nabla y_{\gamma}\right|^{2}}, \nabla v\right\rangle \mathrm{d} x \mid \leq } \\
& \frac{\gamma}{g} \int_{\mathcal{A}_{\gamma}}\left|\frac{\left|\nabla y_{\bar{\gamma}}\right|-\left|\nabla y_{\gamma}\right|}{\bar{\gamma}-\gamma}-\frac{\left\langle\nabla y_{\gamma}, \nabla\left(\frac{y_{\bar{\gamma}}-y_{\gamma}}{\bar{\gamma}-\gamma}\right)\right\rangle}{\left|\nabla y_{\gamma}\right|}\right||\nabla v| \mathrm{d} x \\
& \leq \frac{\gamma}{g|\bar{\gamma}-\gamma|}\left\|\left|\nabla y_{\gamma}\right|-\left|\nabla y_{\bar{\gamma}}\right|-\frac{\left\langle\nabla y_{\gamma}, \nabla\left(y_{\gamma}-y_{\bar{\gamma}}\right)\right\rangle}{\left|\nabla y_{\gamma}\right|}\right\|_{L^{2}\left(\mathcal{A}_{\gamma}\right)}\|v\|_{H_{0}^{1}} .
\end{aligned}
$$

Since the function $|\cdot|: \mathbf{L}^{p}(\Omega) \rightarrow \mathbf{L}^{2}(\Omega)$, with $p>2$, is Newton differentiable, with generalized derivative $G_{|\cdot|}$, given by

$$
G_{|\cdot|}(\varphi)(h)(x):=\left\{\begin{array}{ccc}
\frac{\langle\varphi(x), h(x)\rangle}{|\varphi(x)|} & \text { if } & |\varphi(x)| \neq 0 \\
\left(\varepsilon_{1}, \varepsilon_{2}\right)^{\top} & \text { if } & |\varphi(x)|=0
\end{array}\right.
$$

where $\left|\left(\varepsilon_{1}, \varepsilon_{2}\right)^{\top}\right|<1$, (see [27], Sect. 2.5.1, Thm. 3.48), we obtain that

$$
\left\|\left|\nabla y_{\gamma}\right|-\left|\nabla y_{\bar{\gamma}}\right|-\frac{\left\langle\nabla y_{\gamma}, \nabla y_{\gamma}-\nabla y_{\bar{\gamma}}\right\rangle}{\left|\nabla y_{\gamma}\right|}\right\|_{\mathbf{L}^{2}\left(\mathcal{A}_{\gamma}\right)}=o\left(\left\|\nabla y_{\bar{\gamma}}-\nabla y_{\gamma}\right\|_{\mathbf{L}^{p}\left(\mathcal{A}_{\gamma}\right)}\right),
$$

which, due to Theorem 3.2 implies that

$$
\left\|\left|\nabla y_{\gamma}\right|-\left|\nabla y_{\bar{\gamma}}\right|-\frac{\left\langle\nabla y_{\gamma}, \nabla y_{\gamma}-\nabla y_{\bar{\gamma}}\right\rangle}{\left|\nabla y_{\gamma}\right|}\right\|_{\mathbf{L}^{2}\left(\mathcal{A}_{\gamma}\right)}=o(|\bar{\gamma}-\gamma|)
$$


Therefore, from (A.12) and (A.13), we conclude, for all $v \in H_{0}^{1}(\Omega)$, that

$$
\left|\int_{\mathcal{A}_{\gamma}}\left[\frac{\left|\nabla y_{\bar{\gamma}}\right|-\left|\nabla y_{\gamma}\right|}{\bar{\gamma}-\gamma}-\frac{\left\langle\nabla y_{\gamma}, \nabla \frac{y_{\bar{\gamma}}-y_{\gamma}}{\bar{\gamma}-\gamma}\right\rangle}{\left|\nabla y_{\gamma}\right|}\right]\left\langle\frac{\nabla y_{\gamma}}{\left|\nabla y_{\gamma}\right|^{2}}, \nabla v\right\rangle \mathrm{d} x\right| \rightarrow 0 \text {, as } \bar{\gamma} \downarrow \gamma \text {. }
$$

Therefore, (A.7), (A.9), (A.11) and (A.14) imply (A.6).

Consequently, from (A.5) and (A.6), we obtain that

$$
\lim _{\bar{\gamma} \downarrow \gamma}(\bar{\gamma}-\gamma)^{-1} \mathrm{I}_{1}=g\left(\left(\frac{\nabla \dot{y}_{\gamma}^{+}}{\left|\nabla y_{\gamma}\right|}-\frac{\left\langle\nabla y_{\gamma}, \nabla \dot{y}_{\gamma}^{+}\right\rangle}{\left|\nabla y_{\gamma}\right|^{3}} \nabla y_{\gamma}\right) \chi_{\mathcal{A}_{\gamma}}, \nabla v\right)_{\mathbf{L}^{2}(\Omega)} .
$$

On $\Omega_{4}\left(\lim _{\bar{\gamma} \downarrow \gamma}(\bar{\gamma}-\gamma)^{-1} \mathrm{I}_{4}\right)$ : We recall that $\theta_{\bar{\gamma}}(x)=\theta_{\gamma}(x)=g$ a.e. in $\Omega_{4}$. Thus, the following pointwise equality holds

$$
\frac{\bar{\gamma} \nabla y_{\bar{\gamma}}(x)}{\theta_{\bar{\gamma}}(x)}-\frac{\gamma \nabla y_{\gamma}(x)}{\theta_{\gamma}(x)}=\frac{\bar{\gamma} \nabla y_{\bar{\gamma}}(x)}{g}-\frac{\gamma \nabla y_{\gamma}(x)}{g}=\frac{(\bar{\gamma}-\gamma) \nabla y_{\gamma}(x)+\bar{\gamma} \nabla\left(y_{\bar{\gamma}}-y_{\gamma}\right)(x)}{g},
$$

and thus, since $\mathcal{I}_{\gamma}=\left(\mathcal{I}_{\gamma} \cap \mathcal{I}_{\bar{\gamma}}\right) \cup\left(\mathcal{I}_{\gamma} \backslash \mathcal{I}_{\bar{\gamma}}\right)$, we obtain that

$$
\begin{aligned}
(\bar{\gamma}-\gamma)^{-1} \mathrm{I}_{4}= & \int_{\mathcal{I}_{\gamma}}\left\langle\left[\nabla y_{\gamma}+\bar{\gamma} \nabla\left(\frac{y_{\bar{\gamma}}-y_{\gamma}}{\bar{\gamma}-\gamma}\right)\right], \nabla v\right\rangle \mathrm{d} x \\
& -\int_{\mathcal{I}_{\gamma} \backslash \mathcal{I}_{\bar{\gamma}}}\left\langle\left[\nabla y_{\gamma}+\bar{\gamma} \nabla\left(\frac{y_{\bar{\gamma}}-y_{\gamma}}{\bar{\gamma}-\gamma}\right)\right], \nabla v\right\rangle \mathrm{d} x .
\end{aligned}
$$

Since $\dot{y}_{\gamma}^{+}$is an accumulation point of $\frac{y_{\bar{\gamma}}-y_{\gamma}}{\bar{\gamma}-\gamma}$ in $H_{0}^{1}(\Omega)$ as $\bar{\gamma} \downarrow \gamma$, we conclude that

$$
\int_{\mathcal{I}_{\gamma}}\left\langle\left[\nabla y_{\gamma}+\bar{\gamma} \nabla\left(\frac{y_{\bar{\gamma}}-y_{\gamma}}{\bar{\gamma}-\gamma}\right)\right], \nabla v\right\rangle \mathrm{d} x \rightarrow \int_{\mathcal{I}_{\gamma}}\left\langle\nabla y_{\gamma}+\gamma \nabla \dot{y}_{\gamma}^{+}, \nabla v\right\rangle \mathrm{d} x
$$

On the other hand, from Theorems 2.4 and 3.2, and due to Cauchy-Schwarz and Hölder inequalities, we have that

$$
\begin{aligned}
\left|\int_{\mathcal{I}_{\gamma} \backslash \mathcal{I}_{\bar{\gamma}}}\left\langle\left[\nabla y_{\gamma}+\bar{\gamma} \nabla\left(\frac{y_{\bar{\gamma}}-y_{\gamma}}{\bar{\gamma}-\gamma}\right)\right], \nabla v\right\rangle \mathrm{d} x\right| & \leq\left(\left\|y_{\gamma}\right\|_{H_{0}^{1}}+\bar{\gamma}\left\|\frac{y_{\bar{\gamma}}-y_{\gamma}}{\bar{\gamma}-\gamma}\right\|_{H_{0}^{1}}\right)\|v\|_{H_{0}^{1}\left(\mathcal{I}_{\gamma} \backslash \mathcal{I}_{\bar{\gamma})}\right.} \\
& \leq(K+\bar{\gamma} L)\|v\|_{H_{0}^{1}\left(\mathcal{I}_{\gamma} \backslash \mathcal{I}_{\bar{\gamma}}\right)} .
\end{aligned}
$$

Therefore, from Lemma 3.4 and [2], Lemma A1.16, we conclude that

$$
\int_{\mathcal{I}_{\gamma} \backslash \mathcal{I}_{\bar{\gamma}}}\left\langle\left[\nabla y_{\gamma}+\bar{\gamma} \nabla\left(\frac{y_{\bar{\gamma}}-y_{\gamma}}{\bar{\gamma}-\gamma}\right)\right], \nabla v\right\rangle \mathrm{d} x \rightarrow 0, \text { for all } v \in H_{0}^{1}(\Omega)
$$

Thus, from (A.16) and (A.17), we conclude that

$$
\lim _{\bar{\gamma} \downarrow \gamma}(\bar{\gamma}-\gamma)^{-1} \mathrm{I}_{4}=\left(\left(\nabla y_{\gamma}+\gamma \nabla \dot{y}_{\gamma}^{+}\right) \chi_{\mathcal{I}_{\gamma}}, \nabla v\right)_{\mathbf{L}^{2}(\Omega)} .
$$

On $\Omega_{2}$ and $\Omega_{3}\left(\lim _{\bar{\gamma} \downarrow \gamma}(\bar{\gamma}-\gamma)^{-1} \mathrm{I}_{2}\right.$ and $\left.\lim _{\bar{\gamma} \downarrow \gamma}(\bar{\gamma}-\gamma)^{-1} \mathrm{I}_{3}\right)$ : First, note that

$$
\mathrm{I}_{j}=\int_{\Omega_{j}}\left\langle\frac{\bar{\gamma}}{\theta_{\bar{\gamma}}} \nabla\left(y_{\bar{\gamma}}-y_{\gamma}\right), \nabla v\right\rangle \mathrm{d} x+\int_{\Omega_{j}}\left\langle\frac{\bar{\gamma} \theta_{\gamma}-\gamma \theta_{\bar{\gamma}}}{\theta_{\bar{\gamma}} \theta_{\gamma}} \nabla y_{\gamma}, \nabla v\right\rangle \mathrm{d} x,
$$


for all $v \in H_{0}^{1}(\Omega)$ and $j=2,3$. Next, we analyze the two integrals in (A.19) separately. Thus, since $g \leq \theta_{\bar{\gamma}}(x)$ a.e. in $\Omega$ and due to Theorem 3.2, we have that

$$
\begin{aligned}
\left|\int_{\Omega_{j}}\left\langle\frac{\bar{\gamma}}{\theta_{\bar{\gamma}}} \nabla\left(y_{\bar{\gamma}}-y_{\gamma}\right), \nabla v\right\rangle \mathrm{d} x\right| & \leq \bar{\gamma} \int_{\Omega_{j}}\left|\nabla\left(y_{\bar{\gamma}}-y_{\gamma}\right)\right||\nabla v| \mathrm{d} x \\
& \leq \frac{\bar{\gamma}}{g}\left\|y_{\bar{\gamma}}-y_{\gamma}\right\|_{H_{0}^{1}}\|v\|_{H_{0}^{1}\left(\Omega_{j}\right)} \leq \frac{L \bar{\gamma}|\bar{\gamma}-\gamma|}{g}\|v\|_{H_{0}^{1}\left(\Omega_{j}\right)} .
\end{aligned}
$$

On the other hand, we have that

$$
\begin{aligned}
\left|\int_{\Omega_{j}}\left\langle\frac{\bar{\gamma} \theta_{\gamma}-\gamma \theta_{\bar{\gamma}}}{\theta_{\bar{\gamma}} \theta_{\gamma}} \nabla y_{\gamma}, \nabla v\right\rangle\right| \mathrm{d} x \leq & \int_{\Omega_{j}}\left|\left\langle\frac{(\bar{\gamma}-\gamma)}{\theta_{\bar{\gamma}}} \nabla y_{\gamma}, \nabla v\right\rangle\right| \mathrm{d} x \\
& +\gamma \int_{\Omega_{j}}\left|\left\langle\frac{\theta_{\gamma}-\theta_{\bar{\gamma}}}{\theta_{\gamma} \theta_{\bar{\gamma}}} \nabla y_{\gamma}, \nabla v\right\rangle\right| \mathrm{d} x .
\end{aligned}
$$

Since $g \leq \theta_{\bar{\gamma}}(x)$ a.e. in $\Omega$, and due to the Cauchy-Schwarz inequality, we have that

$$
\int_{\Omega_{j}}\left|\left\langle\frac{(\bar{\gamma}-\gamma)}{\theta_{\bar{\gamma}}} \nabla y_{\gamma}, \nabla v\right\rangle\right| \mathrm{d} x \leq \frac{|\bar{\gamma}-\gamma|}{g}\left\|y_{\gamma}\right\|_{H_{0}^{1}\left(\Omega_{j}\right)}\|v\|_{H_{0}^{1}\left(\Omega_{j}\right)} .
$$

Moreover, since $\gamma\left|\nabla y_{\gamma}(x)\right| \leq \theta_{\gamma}(x)$ and $g \leq \theta_{\bar{\gamma}}(x)$ a.e. in $\Omega$ and due to the Cauchy-Schwarz inequality, we have that

$$
\int_{\Omega_{j}}\left|\left\langle\frac{\theta_{\gamma}-\theta_{\bar{\gamma}}}{\theta_{\gamma} \theta_{\bar{\gamma}}} \gamma \nabla y_{\gamma}, \nabla v\right\rangle\right| \mathrm{d} x \leq \frac{1}{g} \int_{\Omega_{j}}\left|\theta_{\bar{\gamma}}-\theta_{\gamma}\right||\nabla v| \mathrm{d} x
$$

which, from (3.1) and Theorem 3.2, implies that

$$
\int_{\Omega_{j}}\left|\left\langle\frac{\theta_{\gamma}-\theta_{\bar{\gamma}}}{\theta_{\gamma} \theta_{\bar{\gamma}}} \gamma \nabla y_{\gamma}, \nabla v\right\rangle\right| \mathrm{d} x \leq\left(\frac{|\bar{\gamma}-\gamma|}{g}\left\|y_{\gamma}\right\|_{H_{0}^{1}\left(\Omega_{j}\right)}+\frac{\gamma|\bar{\gamma}-\gamma|}{g}\right)\|v\|_{H_{0}^{1}\left(\Omega_{j}\right)} .
$$

Therefore, (A.19), (A.20), (A.21), (A.22), (A.23), Lemma 3.4 and [2], Lemma A1.16, imply that

$$
\lim _{\bar{\gamma} \rightarrow \gamma^{+}} \frac{1}{\bar{\gamma}-\gamma}\left|\mathrm{I}_{2}\right|=\lim _{\bar{\gamma} \rightarrow \gamma^{+}} \frac{1}{\bar{\gamma}-\gamma}\left|\mathrm{I}_{3}\right|=0
$$

Consequently (A.2), (A.15), (A.18) and (A.24) imply the claim.

Acknowledgements. We thank Prof. Dr. Fredi Tröltzsch for helpful comments and for his hospitality during the authors stay in Berlin. We also thank the anonymous referees for many helpful comments which lead to a significant improvement of the article.

\section{REFERENCES}

[1] J. Alberty, C. Carstensen and S. Funken, Remarks around 50 lines of Matlab: short finite element implementation. Numer. Algorithms 20 (1999) 117-137.

[2] H.W. Alt, Lineare Funktionalanalysis. Springer-Verlag (1999).

[3] A. Bensoussan and J. Frehse, Regularity Results for Nonlinear Elliptic Systems and Applications, Applied Mathematical Sciences 151. Springer-Verlag (2002). 
[4] H. Brézis, Monotonicity methods in Hilbert spaces and some applications to nonlinear partial differential equations, in Contributions to Non-linear Functional Analysis, E. Zarantonello Ed., Acad. Press (1971) 101-156.

[5] J.C. De Los Reyes and K. Kunisch, A semi-smooth Newton method for control constrained boundary optimal control of the Navier-Stokes equations. Nonlinear Anal. 62 (2005) 1289-1316.

[6] E.J. Dean, R. Glowinski and G. Guidoboni, On the numerical simulation of Bingham visco-plastic flow: Old and new results. J. Non-Newtonian Fluid Mech. 142 (2007) 36-62.

[7] G. Duvaut and J.L. Lions, Inequalities in Mechanics and Physics. Springer-Verlag, Berlin (1976).

[8] I. Ekeland and R. Temam, Convex Analysis and Variational Problems. North-Holland Publishing Company, The Netherlands (1976).

[9] M. Fuchs and G. Seregin, Some remarks on non-Newtonian fluids including nonconvex perturbations of the Bingham and Powell-Eyring model for viscoplastic fluids. Math. Models Methods Appl. Sci. 7 (1997) 405-433.

[10] M. Fuchs and G. Seregin, Regularity results for the quasi-static Bingham variational inequality in dimensions two and three. Math. Z. 227 (1998) 525-541.

[11] M. Fuchs, J.F. Grotowski and J. Reuling, On variational models for quasi-static Bingham fluids. Math. Methods Appl. Sci. 19 (1996) 991-1015.

[12] R. Glowinski, Numerical Methods for Nonlinear Variational Problems, Springer Series in Computational Physics. SpringerVerlag (1984).

[13] R. Glowinski, J.L. Lions and R. Tremolieres, Analyse numérique des inéquations variationnelles. Applications aux phénomènes stationnaires et d'évolution 2, Méthodes Mathématiques de l'Informatique, No. 2. Dunod (1976).

[14] M. Hintermüller and K. Kunisch, Path-following methods for a class of constrained minimization problems in function spaces. SIAM J. Optim. 17 (2006) 159-187.

[15] M. Hintermüller and K. Kunisch, Feasible and non-interior path-following in constrained minimization with low multiplier regularity. SIAM J. Contr. Opt. 45 (2006) 1198-1221.

[16] M. Hintermüller and G. Stadler, An infeasible primal-dual algorithm for TV-based inf-convolution-type image restoration. SIAM J. Sci. Comput. 28 (2006) 1-23.

[17] M. Hintermüller, K. Ito and K. Kunisch, The primal-dual active set strategy as a semi-smooth Newton method. SIAM J. Optim. 13 (2003) 865-888.

[18] R.R. Huilgol and Z. You, Application of the augmented Lagrangian method to steady pipe flows of Bingham, Casson and Herschel-Bulkley fluids. J. Non-Newtonian Fluid Mech. 128 (2005) 126-143.

[19] K. Ito and K. Kunisch, Augmented Lagrangian methods for nonsmooth, convex optimization in Hilbert spaces. Nonlinear Anal. 41 (2000) 591-616.

[20] K. Ito and K. Kunisch, Semi-smooth Newton methods for variational inequalities of the first kind. ESAIM: M2AN 37 (2003) $41-62$.

[21] J.-L. Lions, Optimal Control of Systems Governed by Partial Differential Equations. Springer-Verlag (1971).

[22] P.P. Mosolov and V.P. Miasnikov, Variational methods in the theory of the fluidity of a viscous-plastic medium. J. Appl. Math. Mech. (P.M.M.) 29 (1965) 468-492.

[23] T. Papanastasiou, Flows of materials with yield. J. Rheology 31 (1987) 385-404.

[24] G. Stadler, Infinite-dimensional Semi-smooth Newton and Augmented Lagrangian Methods for Friction and Contact Problems in Elasticity. Ph.D. thesis, Karl-Franzens University of Graz, Graz, Austria (2004).

[25] G. Stadler, Path-following and augmented Lagrangian methods for contact problems in linear elasticity. J. Comp. Appl. Math. 203 (2007) 533-547.

[26] D. Sun and J. Han, Newton and quasi-Newton methods for a class of nonsmooth equations and related problems. SIAM J. Optim. 7 (1997) 463-480.

[27] M. Ulbrich, Nonsmooth Newton-like methods for variational inequalities and constrained optimization problems in function spaces. Habilitation thesis, Technische Universität München, Germany (2001-2002). 